\title{
2 Temporal response modelling uncovers electrophysiological correlates 3 of trial-by-trial error-driven learning
}

4 Tomas O. Lentz $^{* a}$, Jessie S. Nixon ${ }^{* b}$, and Jacolien van Rij ${ }^{*}$

5 *Author contributions: all authors are equal contributors.

6 aTilburg center for Cognition and Communication (TiCC), Tilburg University, The

7 Netherlands; 'b Quantitative Linguistics Group, Eberhard Karls University of Tübingen,

8 Germany; 'Bernoulli Institute for Mathematics, Computer Science and Artificial Intelligence,

9 University of Groningen, The Netherlands

\author{
ARTICLE HISTORY \\ Compiled April 14, 2022
}

\begin{abstract}
Humans learn from statistical regularities in the environment. There has been increasing interest in recent decades regarding the mechanisms underlying such learning. We used Error-Driven Learning (EDL) to simulate participants' trial-by-trial learning during exposure to a bimodal distribution of non-native lexical tones. This gave us estimates of the degree of expectation of upcoming stimuli over the course of the experiment. The expectation estimates were combined with Temporal Response Function (TRF) fitting to predict the trial-by-trial ERP waveform. The expectation estimates of certain EDL simulations were significantly more informative about the ERP signal than discrete stimulus conditions or overall stimulus probability. These results show that trial-by-trial changes in neural activity can be captured by EDL estimates of expectation. Hence, we tentatively conclude that learning of statistical regularities, as reflected in neural activity, involves continual updating of expectations depending on error-driven learning from cues in the environment.
\end{abstract}

\section{KEYWORDS}

error-driven learning, discriminative learning, Rescorla-Wagner equations, temporal response modelling, event-related potentials, statistical learning

\section{Introduction}

Learning a non-native language requires learning to use acoustic cues differently to the native language. Increasing evidence suggests that listeners learn implicitly from statistical regularities in the environment. However, there is still ongoing discussion about what underlying learning mechanisms are involved. According to error-driven learning theory, learning occurs through a process of prediction and feedback from prediction error: expectations are constantly adjusted in response to incoming information to minimise prediction error (i.e. surprise) over time. The present study investigates whether traces of error-driven learning occur in the trial-by-trial changes in event-related brain potentials (ERPs).

CONTACT Tomas O. Lentz (T.O.Lentz@tilburguniversity.edu), Jessie S. Nixon (jessie.nixon@unituebingen.de), Jacolien van Rij (j.c.van.rij@rug.nl). 
A wide range of studies have demonstrated that humans pick up on statistical regularities in the environment (see Hasson, 2017; Perruchet \& Pacton, 2006; Rebuschat \& Monaghan, 2019, for reviews). Various research traditions - including implicit learning (e.g. Reber, 1989), statistical learning (e.g. Maye, Werker, \& Gerken, 2002; Saffran, Aslin, \& Newport, 1996; Saffran \& Kirkham, 2018), artificial grammar learning (e.g. Trotter, Monaghan, Beckers, \& Christiansen, 2019), and neural adaptation (e.g. Baldeweg, 2007; Frost, Winkler, Provost, \& Todd, 2016) - have focused on different aspects of learning. Perhaps as a consequence of this different focus, these paradigms also differ somewhat in their interpretation of the underlying learning mechanisms involved.

Most research in this area has focused on the question of how the overall summed statistics, such as frequency or co-occurrence probabilities, influence the processing of stimuli. Experimental studies have generally compared behavioural or brain responses in tests before and after a training phase (pre-tests and post-tests; e.g. Wanrooij, Boersma, \& Benders, 2015) or compared post-tests between groups in different training conditions (e.g. Maye et al., 2002; Wanrooij, Boersma, \& van Zuijen, 2014), in order to investigate how participants' performance was influenced by exposure to statistical regularities during training (but see e.g. Costa-Faidella, Grimm, Slabu, Díaz-Santaella, \& Escera, 2011; Koelsch, Busch, Jentschke, \& Rohrmeier, 2016; Nixon, van Rij, Mok, Baayen, \& Chen, 2016). However, the reported effects found in post-tests resulting from manipulations in training conditions are compatible with various different underlying mechanisms. Investigating trial-by-trial changes in the neural signal can shed light on the underlying mechanism, and in particular, the role that prediction error plays in learning. We will return to the details of error-driven learning models below.

In the past, the methods typically used for analysing ERPs have made it difficult to investigate incremental, trial-by-trial learning. ERPs and other neurophysiological measures are generally noisy. They capture responses not only to the experimental stimuli, but also to many other factors, such as muscle movement, regulatory processes, emotional states and so on. Aggregation of measurements within conditions or in preand post-test measurements reduces the influence of these factors, but also leads to loss of information about individual stimuli and individual trials. Trial-by-trial analysis of EEG has been done previously using generalised additive mixed models (e.g. de Cat, Klepousniotou, \& Baayen, 2015; Nixon, 2014; Nixon, van Rij, Li, \& Chen, 2015; Tremblay \& Baayen, 2010). In the present study, however, we use the relatively new statistical technique of temporal-response modelling (Brodbeck, Presacco, \& Simon, 2018; Lalor \& Foxe, 2010) which is able to test how well trial-by-trial predictions capture ERP responses by fitting so-called temporal response functions (TRFs). TRFs link an input signal with continuous measures placed in time to the recorded ERP signal. The present study uses TRFs to investigate whether measures of trial-by-trial expectations derived from error-driven learning models can be used to predict the trial-by-trial EEG signal.

Below in the Background section, we first present the error-driven learning theory and algorithm that we use to simulate the trial-by-trial learning and review the ERP components that may reflect trial-by-trial learning. The EEG data section describes the experimental paradigm and data preprocessing. The Method section provides a brief overview of our methods, from the EDL simulations and TRF models to the statistical tests. We then present in more detail the EDL simulations used to estimate participants' trial-by-trial expectations of the stimuli in the EDL simulations section. The Analysis section shows 1) how TRFs are used to predict the ERP waveforms and 2) how the predicted ERP waveforms are tested against the observed ERP data. The 
Results section presents the informative models and visualises how these models relate to the ERPs. Finally, in the Discussion, we examine what our results can tell us about trial-by-trial learning, the learning representations and cognitive tasks involved in this experiment and the timing and scalp distribution of the effects found in the ERPs. We also discuss the implications for learning and the potential benefits of our modelling method for future research.

\section{Background}

\subsection{Error-driven learning (EDL)}

Two main classes of learning algorithms have been proposed in the literature: generative models and discriminative models. The aim of generative models is to determine the underlying population distribution from a sample distribution. Generative models include distributional learning models and statistical models that are based on statistical measures, such as frequency or co-occurrence probability. Generative models have often been used in language acquisition and linguistics research (e.g. Maye et al., 2002; Saffran et al., 1996; Saffran \& Kirkham, 2018). In contrast, error-driven learning models are discriminative (Bröker \& Ramscar, 2020; Hoppe, Hendriks, Ramscar, \& van Rij, 2022; Ramscar, Dye, \& McCauley, 2013; Ramscar \& Yarlett, 2007; Ramscar, Yarlett, Dye, Denny, \& Thorpe, 2010). Discriminative learning models have a strong connection to information theory: Shannon (1948) described the problem of communication as selecting the appropriate intended message from a finite set of possible intended messages. Likewise, discriminative error-driven learning models use incoming information (cues) to select or discriminate between a set of possible outcomes. In the context of communication, these outcomes could be intended messages or parts thereof.

In discriminative, error-driven learning theory, rather than simply acquisition of knowledge, learning is considered to be a process of reduction of uncertainty (Baayen, Milin, Đurđević, Hendrix, \& Marelli, 2011; Ramscar et al., 2013, 2010). To this end, potentially any discriminable cues in the environment can be used to predict important outcomes. If certain cues are repeatedly informative for predicting the occurrence (or non-occurrence) of an outcome, then over time encountering such cues will lead to an expectation of the occurrence (or non-occurrence) of said outcome.

Crucially, discriminative error-driven learning models involve cue competition (see Hoppe et al., 2022; Nixon, 2018, 2020; Ramscar et al., 2010, for further discussion). If multiple cues precede an outcome, each of these cues contributes to the expectation of the outcome: formally, each cue-outcome connection has a connection weight; the expectation or activation of an outcome is the sum of weights of all present cues. Weight adjustment is shared equally between all present cues: when an outcome occurs, the connection weights from all present cues to the outcome are increased with the same value; when the outcome does not occur connection weights decrease with the same value. Thus, cues compete for relevance in predicting outcomes. Importantly, however, the weight adjustment also depends on how much the present cues lead the learner to expect a particular outcome, based on previous learning. More learning occurs when an unexpected outcome occurs or when an expected outcome does not occur, compared to when an expected outcome occurs or an unexpected outcome does not occur. Thus, current learning depends on expectations developed over the course of previous learning. The next section presents the algorithm used for the error-driven learning simulations. 


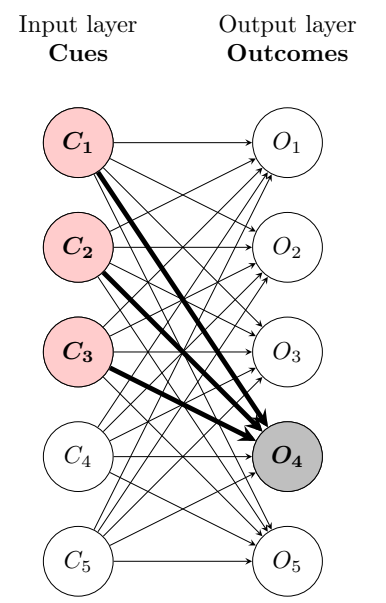

(a)

\begin{tabular}{lrrrrr}
\hline & \multicolumn{5}{c}{ Outcomes } \\
\cline { 2 - 6 } Cues: & $O_{1}$ & $O_{2}$ & $O_{3}$ & $\boldsymbol{O}_{4}$ & $O_{5}$ \\
\hline $\boldsymbol{C}_{\mathbf{1}}$ & 0.024 & 0.042 & 0.076 & $\mathbf{0 . 0 7 3}$ & 0.058 \\
$\boldsymbol{C}_{\mathbf{2}}$ & 0.017 & 0.061 & 0.110 & $\mathbf{0 . 0 9 3}$ & 0.053 \\
$\boldsymbol{C}_{\mathbf{3}}$ & 0.016 & 0.067 & 0.073 & $\mathbf{0 . 0 8 5}$ & 0.045 \\
$\boldsymbol{C}_{4}$ & -0.005 & 0.093 & 0.116 & 0.101 & 0.015 \\
$\boldsymbol{C}_{5}$ & 0.019 & 0.076 & 0.109 & 0.086 & 0.035 \\
\hline
\end{tabular}

(b)

Figure 1. Example of EDL single layer network with cues and outcomes (a) and the associated matrix of connection weights (b). The red colored cues in (a) - $C_{1}, C_{2}, C_{3}$ - represent the present cues; the connections from all present cues to outcome $\mathrm{O}_{4}$ (thick lines) are summed to derive the activation for outcome $\mathrm{O}_{4}$, which reflects the strength of expectation (i.e., the prediction) that this outcome will be observed given the present cues. The matrix of connection weights reveals that the activation of $O_{4}$ is 0.251 (i.e., the sum of the bold numbers).

\subsubsection{The Rescorla-Wagner learning equations}

In the present study, the EDL simulations provide an estimate of how expected the incoming sound was on each trial. As discussed above, these expectations are based on all present cues and, through trial-by-trial adjustments, develop over the course of learning. The simulations are based on the Rescorla-Wagner learning equations (Rescorla \& Wagner, 1972; Widrow \& Hoff, 1960/1988). This model consists of a simple feedforward two-layer network, fully connected and without any hidden layers. The nodes in the input layer represent the cues, observed events or information that elicits a prediction about upcoming events. The nodes in the output layer represent the outcomes, that is, the upcoming events or information. The connection weights $w$ represent the prediction strength from individual cues to outcomes. The network can be represented by a matrix of connection strengths, as illustrated in Figure 1. The connection strengths between cue $i$ and outcome $j$ are incrementally updated at each observed event (trial) $t$, for all present cues and all outcomes, as described in Definition 2.1a.

Definition 2.1. Specification of error-driven learning (EDL) 
a. Update of connection weight matrix:

$V_{i j}^{t+1}=V_{i j}^{t}+\Delta V_{i j}^{t}$

with $\mathrm{V}$ being the matrix of connection strengths.

b. Learning rule for updating $V_{i j}$ :

The connections between cues and outcomes are updated at each event following the learning rule proposed independently by Rescorla and Wagner (1972) and Widrow and Hoff $(1960 / 1988)$, specified in Definition 2.1b. In this learning rule, the update of weights is determined by three factors: (1) presence or absence of cues and outcomes, (2) the parameters $\eta$ and $\lambda$, and (3) current activation, which is the sum of connection weights from all present cues to a given outcome.

Firstly, Equation 2.1b describes the change in weights for three different cue-outcome combinations: (i) for all cues present on the current trial, connection weight increases to all present outcomes; (ii) for all cues present on the current trial, connection weight decreases to all absent (but previously encountered) outcomes; (iii) for any cues not present during the current trial, no adjustment is made. Additionally, for outcomes not encountered previously, no adjustment is made. Importantly, in (i) and (ii) adjustment to cue weights is divided equally among all present cues, which creates cue competition.

Secondly, the parameters determine the speed of learning. The parameter $\eta$ is the learning rate $^{1}$, set to 0.01 . The parameter $\lambda$ is the asymptote of the activation, which is typically (and in the present study) set to 1 .

Finally, and critically, as mentioned in the previous section, when weights are adjusted, the amount of adjustment depends on previous learning. The activation of an outcome $j$ is defined as the sum of connection weights from all present cues to outcome $j$ (Definition 2.1c; see Figure 1a for an illustration). The weight update $\Delta V_{i j}$ depends on the activation. Higher activation values indicate a stronger prediction that outcome $j$ will occur. For high activation values, the prediction error is small when outcome $j$ is actually observed, but large when $j$ is not observed. The learning rule in $2.1 \mathrm{~b}$ aims to reduce prediction error over time. Therefore, the calculation of the weight update involves subtracting the activation from $\lambda$ (and multiplying by the learning rate) when outcome $j$ is observed, resulting in small updates for high activation values and large updates for low activation values. When outcome $j$ is not observed, calculation of the weight update involves subtracting the activation from 0 (and multiplying by the learning rate), which decreases connection strength. The decrease in connection strength is large for high-activation (expected) outcomes and small for low-activation (unexpected) outcomes.

\footnotetext{
${ }^{1}$ The Rescorla-Wagner equations allow for a separate parameter for the salience of cues, salience of occurring outcomes and salience of non-occurring outcomes. In the present study, we reduce these three model parameters to one, the learning rate.
} 
Because the connection weights are updated based on the current activation and the observed information, the model does not need to remember the complete history of co-occurrences to capture statistical patterns in the input. Interestingly, this simple learning model can explain a wide range of cognitive and linguistic phenomena, such as order effects in language (e.g., Hoppe, van Rij, Hendriks, \& Ramscar, 2020; Nixon, 2018, 2020; Ramscar et al., 2010), the Kamin blocking effect (e.g., Kamin, 1969; Nixon, 2020), learning about predictive relations in the environment (Nixon, Poelstra, \& van Rij, 2022), distributional learning (Olejarczuk, Kapatsinski, \& Baayen, 2018) and first language speech sound acquisition (Nixon \& Tomaschek, 2020, 2021). We refer to Hoppe et al. (2022) for a thorough review of the dynamics of EDL and the phenomena it has explained and Nixon (2020) for comparison with other leading learning models. Here we will focus on how EDL models are used to simulate the online learning of non-native lexical tones.

\subsection{ERP components related to prediction and learning}

The ERP signal is derived from electro-encephalogram (EEG; voltage) measurements that are time-locked to an event (hence 'event-related' potentials), usually the presentation of a stimulus. Various ERP components have been found to reflect learning, adaptation and predictive processing. To facilitate interpretation of the results, we briefly summarise the candidate components, namely the mismatch negativity response, the P3, error negativity and the N400. They are divided into early and late components, in relation to the time-windows in our analysis.

\subsubsection{Early components}

The mismatch negativity component (MMN; e.g., Näätänen, Gaillard, \& Mäntysalo, 1978; Näätänen \& Kreegipuu, 2011) occurs when the brain detects a change in auditory stimuli, usually in the context of the oddball paradigm. In this paradigm, occasional deviant stimuli are presented within a sequence of a large number of standard stimuli. In response to deviant stimuli, the MMN occurs between 100-250 ms after stimulus onset, starting at the N1, which peaks around $100 \mathrm{~ms}$, and overlapping with the P2, which peaks around 180-200 ms (see Näätänen \& Kreegipuu, 2011, for a review). Because of the overlap with other components, the MMN is often (but not always) reported as the difference in the ERP signal between standard and deviant stimuli.

The MMN seems to capture violation of predictions and as such it may be an index of learning. The standard-deviant ratio influences the magnitude of the MMN, with a higher number of deviants reducing the MMN magnitude (e.g., Haenschel, Vernon, Dwivedi, Gruzelier, \& Baldeweg, 2005). Koelsch et al. (2016) report that the occurrence and magnitude of the MMN depend on the conditional probability of a stimulus given the earlier two sounds, with low probability sounds eliciting the largest MMN magnitude.

The MMN has been used to investigate the learning of (non)native speech sounds. Because a difference between standards and deviants is only found in the ERPs when participants can detect the difference between standard and deviant stimuli, the MMN can be used to detect whether participants can discriminate between speech sounds. For example, after training with nonnative vowel contrasts, an MMN response may occur to the deviant vowels which were presumably not discriminated before training (e.g., Wanrooij et al., 2014; Winkler et al., 1999). 


\subsubsection{Late components}

The $P 3$ is a positive-going ERP component that is also associated with expectation and surprise (e.g., Polich, 2011; Rushby, Barry, \& Doherty, 2005). Like the MMN, the P3 has often been investigated using an oddball paradigm. Two subcomponents of the P3 have been identified, namely the P3a and the P3b. The P3a occurs around 300-500 ms post-stimulus (Kopp \& Wolff, 2000) and occurs with or without conscious attention to the critical stimuli (Polich, 1988; Snyder \& Hillyard, 1976). The P3b occurs around 500-700 ms post-stimulus (Kopp \& Wolff, 2000) and requires attention to the target (Snyder \& Hillyard, 1976). The P3a is distributed over the central and parietal electrodes, while the P3b is distributed more parietally (Polich, 2011).

The error negativity $\left(\mathrm{N}_{\mathrm{E}}\right.$; also called error-related negativity; ERN) is usually associated with overt responses. It is a negative-going component that peaks around $80 \mathrm{~ms}$ after participants make an incorrect response, but starts prior to the response (Falkenstein, Hohnsbein, Hoormann, \& Blanke, 1991; see Holroyd, Nieuwenhuis, Mars, \& Coles, 2004, for a review). In addition to this response $N_{E}$, Miltner, Braun, and Coles (1997) discovered that a similar $\mathrm{N}_{\mathrm{E}}$ component also appeared when participants received negative feedback. Holroyd et al. (2004) argue that the $\mathrm{N}_{\mathrm{E}}$ is elicited when a prediction error is detected: if a participant makes an error and detects it prior to receiving feedback, an $\mathrm{N}_{\mathrm{E}}$ appears. In contrast, when the participant does not detect the error at the time of the response, then negative feedback elicits a feedback $\mathrm{N}_{\mathrm{E}}$ at around $250 \mathrm{~ms}$ after the onset of the feedback stimulus.

The $N_{400}$ (Kutas \& Hillyard, 1980) is a negative-going waveform that peaks at around $400 \mathrm{~ms}$ (see e.g., Kutas \& Federmeier, 2011, for a review). N400 amplitude is greater in centro-parietal sites in response to surprising or incongruent events compared to expected events. The functional scope of the N400 is complex, but seems to relate to higher-level expectation or prediction, particularly in semantic processes. Several studies have failed to find $\mathrm{N} 400$ effects for lower-level surprising events, such as an orthographic switch to capital letters (Kutas \& Hillyard, 1980) or notes that violated musical expectations (Besson \& Macar, 1987). Increased N400 amplitudes have been found in response to surprising compared to expected items in word lists, sentence processing and broader discourse processing, as well as visual scenes in videos (e.g., Kutas \& Hillyard, 1980; Nieuwland \& van Berkum, 2006; Sitnikova, Kuperberg, \& Holcomb, 2003).

\subsection{Research questions and hypotheses}

Previous research using the oddball paradigm has tested whether the ERP signal differs between discrete stimulus conditions, usually standard vs. deviant. In the present study, we address whether continuous trial-by-trial learning leaves traces in the ERP signal. In particular, we were interested in whether participants use cues encountered during the experiment to develop expectations about the occurrence of particular stimulus conditions (outcomes) on each trial. Furthermore, we also explored which input representations (cues and outcomes) are predictive of brain activity and which components of the ERP are involved. Therefore, the present study addresses three main questions.

Research question 1: Do participants' neural signals show evidence of trial-by-trial learning? We will test this question by investigating whether the activation values for stimuli, derived from the EDL simulations, contain information about participants' neural signals. Specifically, we compare the predictions of our EDL simulations to 
control models based on a) discrete stimulus conditions and b) stimulus probabilities. Such models represent equivalent information bar the trial-by-trial learning simulated using the EDL paradigm. The hypothesis we test is that at least one EDL simulation is more informative about the ERP signal than control models (a) and (b).

Research question 2: What do participants learn in this task? In other words, which input representations (cues and outcomes) allow simulations that capture participants' trial-by-trial learning? We hypothesise that two main types of learning occur. Firstly, we expect that participants might use characteristics of each incoming stimulus to group the stimuli into categories, such as high vs. low lexical tone or standard vs. deviant. Secondly, we expect that participants might use information about the sequences of stimuli to predict upcoming stimuli. We will test these hypotheses by simulating both category learning and predictive sequence learning using various cue-outcome structures. These simulations are tested against control models as outlined for Research question 1; we hypothesise that at least one simulation of each type yields an input signal that is more informative about the ERP signal than a control model.

Research question 3: How does trial-by-trial learning as modelled by the EDL simulations modulate the timing, location, and shape of the ERP waveforms? To answer this question, we divide our analysis into two time windows, early (0-350 ms) and late (250-600 ms). The analysis also yields information about the scalp distribution as it is performed for each electrode independently. Specifically, we explore how the trial-by-trial modulation of the ERP waveform relates to the EDL simulations: Do different EDL simulations modulate the ERP waveforms differently? And vice versa, do EDL simulations that show a similar modulation of the ERP waveforms share input representations?

As explained in more detail below, we test the extent to which EDL simulations can be linked to ERP signals by using them as input for TRFs. The resulting prediction is hypothesised to be more informative than baseline models that only capture static properties of the stimuli, including a stimulus' overall probability. If this hypothesis is confirmed, it answers our first question whether participants' neural signals show evidence of trial-by-trial learning. To answer our second question, we implement fifteen EDL simulations with various cue-outcome structures. If only one of the EDL-derived models is significantly more informative than the baseline models, we take this as evidence for trial-by-trial learning in the neural signal in the way this informative EDL simulation produces its estimates. We correct our statistical tests for the number of models.

\section{EEG data}

EEG data were recorded as participants listened to tokens from a 13-step pitch continuum of the syllable/li/. The syllable (duration $285 \mathrm{~ms}$ ) was recorded by a female speaker, then resynthesised using Praat (Boersma \& Weenink, 2014). The continuum was based on Cantonese mid-level and high-level tones (hereafer, for simplicity, the tones are referred to as 'low' and 'high' tones, respectively). Presentation order was adapted from the oddball paradigm: A series of four standards was presented, followed by one deviant. However, instead of using identical stimuli for all standards and all deviants, the stimuli were sampled from a bimodal distribution with the mid and high tones each represented by a roughly Gaussian distribution of tokens 1-6 and 8-13, respectively (see Figure E1 in Appendix E). The experiment consisted of eight blocks of 42 trial sets, where each trial set consisted of five trials: four standards and one deviant (336 trial sets; 1680 trials). The standard was consistent within blocks (either the low 
Odd block (Ba)

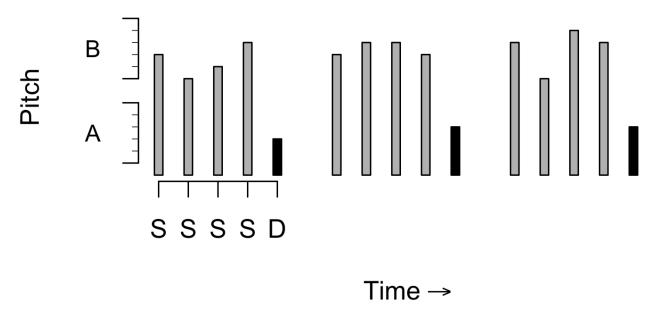

Even block (Ab)

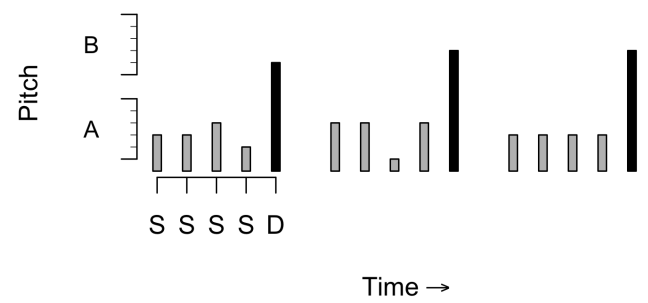

Figure 2. Examples of three trial sets in the odd blocks (standards of type B, high tone; deviants of type A, mid tone), left panel) and in the even blocks (standards of type A, mid tone; deviants of type B, high tone, right panel): each trial set consisted of four standard stimuli followed by one deviant. The stimulus sounds differed only in pitch (y-axis) and were sampled from a bimodal pitch continuum of 13 steps. ' $\mathrm{S}$ '=standard, 'D'=deviant.

or high tone) and alternated between blocks. The inter-stimulus interval was varied randomly between $600-800 \mathrm{~ms}$. The experimental design is illustrated in Figure 2 .

EEG data were collected from 20 right-handed, native German-speaking participants, four of whom were excluded due to excessive noise, leaving data from 16 participants. EEG was recorded from 32 electrodes in the standard 10-20 system. If there was too much noise in a channel during a trial, the channel was excluded for that trial (4.4\%) and Independent Component Analysis (ICA) in Fieldtrip (Oostenveld, Fries, Maris, \& Schoffelen, 2011) was used to exclude blinks and ocular artefacts. We filtered the data between $0.1 \mathrm{~Hz}$ and $15 \mathrm{~Hz}$. The grand averages of electrodes FC6 and $\mathrm{Pz}$ are included in Appendix A as an illustration. The voltages in each channel were transformed to z-scores (per participant and channel).

Preliminary analysis suggests a mismatch negativity response (Boll-Avetisyan et al., 2018). The EEG experiment was followed by two offline behavioural tasks, which will not be discussed here (see Nixon et al., 2018).

\section{Method}

In the present study, EDL simulations yield estimates of how expected the incoming stimulus is for each participant on each trial. The specification of these simulations is described in more detail in Section 5. These expectation estimates provide a continuous measure, activation, rather than categorising each trial as expected or not. The present paper investigates whether EDL activation allows for better prediction of the ERP signal than two types of control models: (1) baseline models, based on the traditional ERP analysis, namely a binary categorisation of trials based on stimulus types, such as standard vs deviant or high vs low tone category, and (2) probability models, based on probability scores of each pitch stimulus over the whole experiment.

Schematic representations of the traditional ERP analysis method and our analysis method using TRF fitting are shown in Figure 3. In essence, TRF fitting shares with traditional ERP analysis that TRFs represent the change in the EEG signal over time in a window after stimulus presentation. The key difference is that estimates are fitted based on a range of expectedness (activation) values, rather than by comparing conditions or subtracting one condition from another. Details of the TRF modelling process are presented in Section 6. A TRF analysis with two stimulus conditions, such 
as standard vs. deviant (shown in diagram in Figure $3 a$ ), can be seen as a special case of our model (shown in diagram in Figure 3b), in which all trials are classified as either fully unexpected or fully expected.

Our analysis procedure involves three statistical tests (Figure 4 gives an overview), which are described in more detail in Section 6. For each EDL simulation, we test the informativity of fitted TRFs as models of the process that generated the measured ERP waveform. The activation values obtained from the EDL simulations can be seen as a scaled ranking of all trials by expectedness, and informativity about the underlying process entails that the ranking corresponds to one or more ERP components that (linearly) increase or decrease along with the ranking. Hence, the more informative this ranking is about the underlying process, the more informative the predictions made by the TRFs will be about the ERP data. In traditional ERP analysis, it is possible to statistically compare the difference between the two categories of EEG recordings, thus testing whether the ERP difference wave is different from zero. However, for a TRF such a comparison is not directly possible. A TRF may not be zero because of components that are always present, irrespective of the expectedness of a trial. In addition, our EDL simulations, though designed to capture learning, may potentially lead to rankings that also reflect other properties of the trials. To address both issues conservatively, our statistical testing involves comparing our EDL-derived models to a set of control models (see Section 6 for more detail).

The EDL simulations in the present study capture the process of using cues to learn to discriminate the outcome stimuli. If the EDL simulations capture variation in the ERP signal, we need to ensure that this is not (only) due to the EDL activations capturing differences between the outcome stimuli, but rather due to trial-by-trial learning of the stimuli. In the simulations where there are only two different outcome types (i.e., 'standard', 'deviant' or 'high', 'low'), baseline models are created in which the TRFs are based on a simple division of all trials into the two stimulus conditions, based on the outcome type. These baseline models are conceptually similar to comparing stimulus conditions in traditional ERP analysis, although here we do it with TRF modelling. Test 1 comprises comparing the EDL models against these baselines. If the EDL models are more informative than their baselines, the continuous activation measure - a measure of expectation on each trial - is more informative about the neural response than discrete stimulus conditions based on static properties of the stimuli.

One group of EDL simulations (the '-val' simulations, see Table 2, Section 5) learns to predict the individual steps on the pitch continuum. With this type of set-up, it is not meaningful to create a baseline model like those described above, because there are multiple (12) continuum steps, but no expected ordered effect of the continuum step - we do not expect a larger ERP response to higher values, for example. Therefore, in Test 2, we compare these models to a model that is not based on the outcome (the pitch value), but based on the probability of the outcome. Unlike the pitch value itself, the probability of occurrence of the pitch values does have an order that we might posit to affect participants' expectation: if stimulus probability has an effect, higher probability stimuli are more expected. Hence, a model using only probabilities as input, but not trial-by-trial estimates of learning, is a good model for comparison with the 'val' simulations.

In addition to providing a comparison for the val models, these probability models also represent a theoretical model of learning. As discussed in the Introduction, distributional learning models posit that people learn from the frequency distribution of stimuli (e.g. Maye et al., 2002). According to these models, expectations should differ depending on stimulus probability. Therefore, the other EDL simulations (the HL and 


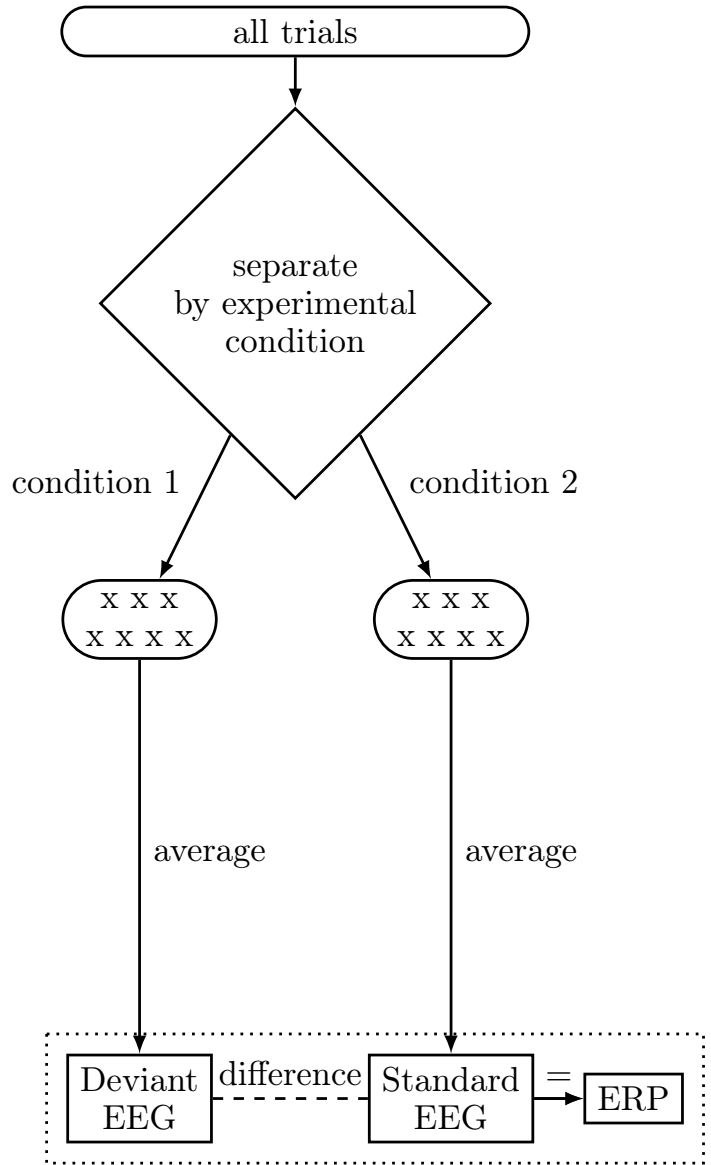

(a) Traditional ERP analysis

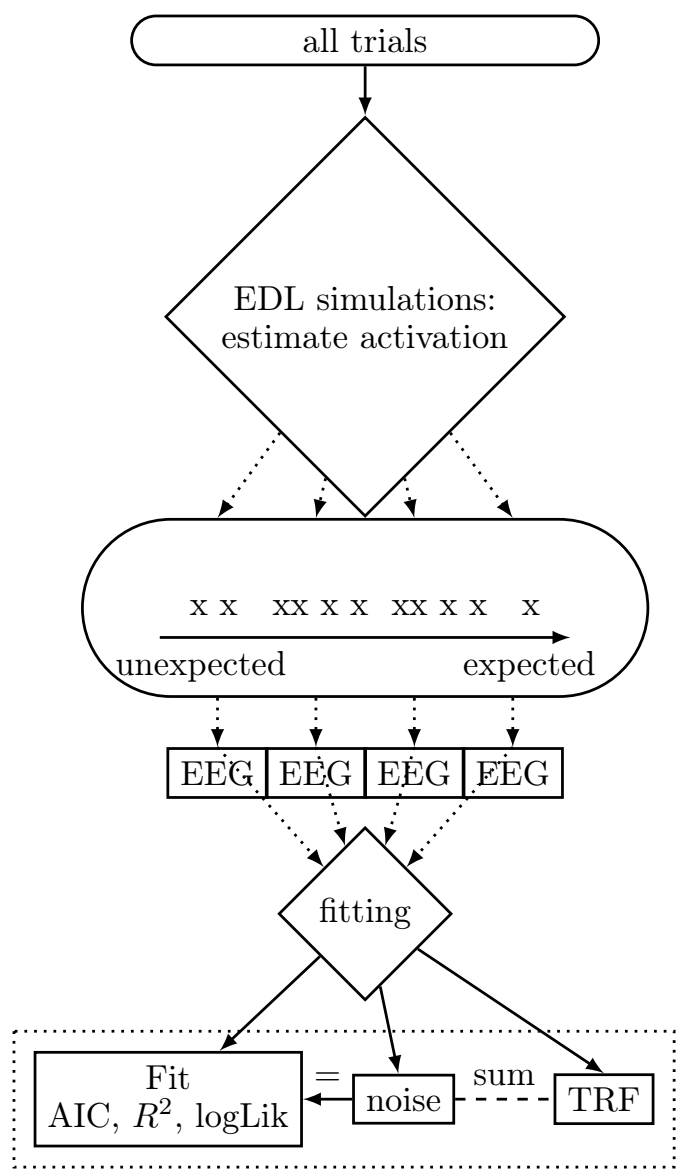

(b) TRF derivation using EDL simulations

Figure 3. Diagram comparing data treatment for traditional ERP analysis vs. the TRF approach, as in the present study. Left: In the classic ERP setup, trials are separated into (usually two) stimulus conditions (e.g 'standard' vs. 'deviant' or 'high tone' vs. 'low tone'). The EEG signal is averaged and these averages are compared to obtain the ERP difference wave, marked in the diagram as 'ERP'. Right: In our study, error-driven learning (EDL) simulations are run, yielding an estimate of the expectedness of the stimulus presented on each trial; the estimates are continuous values on an arbitrary scale. A function is fitted using brute-force, which captures the component in the EEG that gradually increases with the expectedness. Instead of a difference score, the result is a predicted response signal and a TRF fitted as closely as possible to the ERP for each trial. The quality of the fit allows us to estimate how informative the simulation (i.e. the expectation estimate) was. The TRF can be inspected to see the effect of activation on the ERP signal, such as the timing and polarity of the effect. 


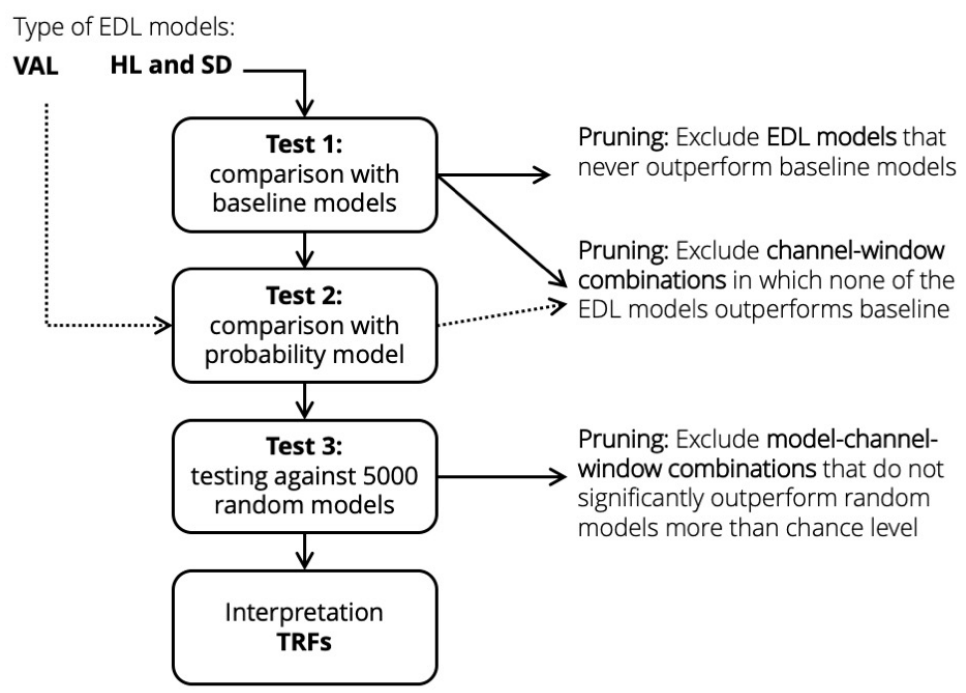

Figure 4. Overview of statistical tests and pruning steps. Note that the probability model acts in place of the baseline model for the EDL '-val' simulations. Therefore Test 1 does not apply and Test 2 is used for pruning these models, but not for pruning the other EDL simulations.

SD models) are compared both to their own baseline (Test 1) and to the probability model (Test 2). Comparison to the probability model allows us to test whether the EDL models are more informative about the ERP signal than a model based on the statistical distribution of stimuli. The informativeness of each TRF, as used in Tests 1 and 2, is captured by its AIC value, as will be described in more detail in Section 6 . A summary of each individual test is provided below. There are three tests, which are mostly independent tests. However, in rare cases, we conducted pruning based on Test 1 , see footnote. ${ }^{2}$. In general, if a model-channel-time window combination did not pass Test 1 , it was not pruned, but instead still passed to Test 2 and/or $3 .{ }^{3}$

Test 1. Our analysis begins by testing each EDL model against its baseline model in each channel in each time window. Test 1 determines whether we are able to find evidence of trial-by-trial learning as captured by our EDL simulations, rather than only differences based on static properties of the stimuli. Technically, we perform a repeated test of the main hypothesis that for at least one channel in at least one time window at least one EDL model leads to a TRF model that is more informative than its baseline. Because the baseline model captures the temporal alignment and the static properties of the outcome stimuli, this means that if the EDL-derived TRF only fits the data due to these two factors, it will not pass Test 1.

Test 2. Our second test compares the EDL models to a probability model, which is a TRF based on the overall probability of the stimuli. As mentioned above, the 'val' models are not compared to a baseline model based on their outcome stimuli like the other EDL models, but are instead compared to the probability model. In addition, each EDL model that passed Test 1 is also compared to the probability model in each remaining channel in each time window. Test 2 determines whether we are able to find

\footnotetext{
${ }^{2}$ Pruning only took place in two specific cases: a) channel-time window combinations were pruned if no EDL model was more informative than its baseline in that channel-time window combination and b) EDL models were pruned if they were not more informative than their baseline in any channel-time window combination. This led to only one case of pruning under a).

${ }^{3}$ We thank an anonymous reviewer for making suggestions that lead us to include this test.
} 
evidence that trial-by-trial learning as captured by our EDL simulations outperforms a model based on the statistical distribution of the stimuli. Technically, we perform a repeated test of the main hypothesis that for at least one channel in at least one time window at least one EDL model leads to a TRF model that is more informative than the probability model. In summary, the results of Test 2 allow us to determine which of the EDL models that passed Test 1 are also more informative than the probability model and in which channels in each time window. In addition, it also allows us to test the '-val' simulations against a model with access to temporal information.

Test 3. Finally, for each combination (time window, channel) and for each EDLderived model that beat its baseline, as well as for the val models that beat the probability model, an additional safety check was performed, comparing each individual EDL model in each channel in each time window to a set of random models.

By using conservative tests in Tests 1 and 2, we have confidence that, if any EDLderived model passes the test(s), that EDL-derived TRFs may captures actual variance in the EEG data corresponding to our continuous activation measure - how expected the stimuli are - not just due to differences between stimulus types or the statistical distribution of stimuli. Because there is a range of activation values in the different simulations, while the trials can only be ranked in a limited number of ways, it is possible that a large enough selection of rankings accidentally contains one or more rankings that explains some variance. As estimating the number of meaningfully different rankings is difficult, we perform a simple, non-parametric test for each combination of EDL simulation, channel and time window not pruned in Tests 1 and 2: We test the hypothesis that the EDL simulation beats a randomly generated model more often than expected by chance. The activation values generated by all EDL simulations were simply randomly assigned, one to each trial, to create 5000 different random input signals, which are still time-aligned with the trials. These random input signals were then used to fit TRFs. The amount of variance explained by the random models was compared to that of each EDL-derived model. If the latter beat the random models (i.e., had a higher amount of explained variance), more often than expected by chance (i.e., significantly more often than $50 \%$ of the comparisons), we consider the EDLderived model to have passed Test 3; the test is performed per channel/time window combination.

\section{EDL simulations: obtaining trial-by-trial estimates of participants' level of expectation}

As described in Section 2.1, we use EDL simulations to generate an estimate of participants' expectation of an upcoming stimulus; in the TRF derivation overview shown in Figure 3 the simulations are placed near the top, as a diamond shape. We generated one measure per participant per trial as an estimate of the participant's expectation of the category (categorisation simulations) or upcoming stimulus (prediction simulations). This measure was the activation; that is, the sum of weights from all present cues to the outcome stimulus that occurred on the present trial. As each participant was exposed to a different sequence of stimuli, we simulated learning for each participant separately.

Determining the appropriate cues and outcomes as representation of the learning process is not trivial (see Bröker \& Ramscar, 2020, for discussion). Furthermore, we assume that all discriminable cues might potentially be used to predict important events. This means that learning may occur on multiple levels simultaneously. There- 
fore, the simulations investigated two types of cognitive task, four possible cue types (i.e. the information participants used to predict the outcomes) and three possible outcome types (the event that participants learned to categorise or predict).

\subsection{Model specification: Cognitive task, cues and outcomes}

The two types of cognitive task we investigated were categorisation (Table 1) and predicting the upcoming stimulus in the sequence (Table 2). In the categorisation simulations, various aspects of an incoming stimulus were used to categorise the same stimulus. That is, the cues and outcome were the same auditory stimulus. This is in contrast to the predictive models, in which various cues were used to predict an upcoming stimulus; the cues and outcome were different stimuli.

The reason for our choice of these two learning tasks is as follows. In terms of the categorisation task, the oddball design of the experiment (a series of standards followed by a deviant) provides structure that may lead to learning of standard vs. deviant or high vs. low tone categories. That is, the fine-grained acoustic differences may be downweighted with an increase in discrimination of the large-scale differences in lexical tone. In terms of the predictive simulations, on the other hand, previous EDL studies have shown that temporally earlier stimuli are used to predict temporally later stimuli (Hoppe et al., 2020; Nixon, 2018, 2020; Nixon \& Tomaschek, 2020, 2021; Ramscar et al., 2010). The sequential presentation of stimuli in this experiment is likely to lead to learning through prediction of upcoming stimuli.

The cues used in the simulations were as follows. The tone bigram cues represent a sequence of cues. This is the only simulation type in which the input cues on each trial include information from more than one trial. Participants learn from a sequence of stimuli, namely a sequence of three overlapping lexical tone bigrams. Bigrams are created with a moving window over the stimulus sequence. For example, the stimulus sequence high high high low produces the bigram cue sequence $\mathrm{HH}$ HH HL. The pitch value cues were the pitch value steps along the continuum (1-6, 8-13). As described in Section 3, the acoustic stimuli presented to participants were sampled from a pitch continuum of Cantonese tones. In a discrimination task, the participants were usually not able to detect differences of two steps along the continuum (although finegrained effects were found in a continuous pitch estimation task; Nixon et al., 2018); therefore, the cues representing each pitch value were a window of three continuum steps. This added a 'fuzziness' or lower perceptual acuity to the learning, to account for the low sensitivity to single steps on the continuum. For example, if the stimulus presented on a particular trial was continuum step 4, the three input cues to the simulation would be 3,4 and 5. The pitch difference cues are the number of steps on the continuum between the pitch value of the previous and current stimulus. For example, if the previous stimulus was 5 and the current stimulus 3 , the pitch difference would be -2 . The window of three is also applied to the pitch difference cues. Thus, in this example, the input cues would be $-3,-2$ and -1 . The position cues represent the position of the current trial in the trial set. As described in Section 3, the trial sets consisted of five trials, of which there were four standards followed by one deviant. The letters A-E represent the positions 1-5.

The outcomes used in the simulations were as follows. The outcomes were either high vs. low lexical tone; standard vs. deviant stimulus; or pitch values. The pitch value outcomes were the same as above for the pitch value cues, except that they were a single value, not a window of three. 
Table 1. Categorisation simulations. Overview of the different error-driven learning simulations in the categorisation analysis. For categorisation, the cue is always the same stimulus as the outcome. For example, a presented stimulus might be pitch value 10. In the Cat-val-HL simulation, the cues would be a window around $10\left(' 9 \_10 \_11^{\prime}\right)$, the present outcome would be the lexical tone High and the absent outcome would be the lexical tone Low. The simulation learns to categorise Pitch 10 as High or Low. Pitch diff $=$ Pitch difference, i.e. the change in pitch relative to the previous trial.

\begin{tabular}{|c|c|c|c|}
\hline Simulation & Cue type & Possible input cues & Outcomes \\
\hline Cat-val-HL & $\begin{array}{l}\text { pitch value, } \\
\text { window of } 3\end{array}$ & $\left\{{ }^{\prime} 1 \_{ }^{\prime},{ }^{\prime} 1{ }_{-}{ }^{2} \_3^{3}, \ldots,{ }^{\prime} 12 \_13^{\prime}\right\}$ & $\begin{array}{l}\text { \{'Low', } \\
\text { 'High'\} }\end{array}$ \\
\hline Cat-diff-HL & $\begin{array}{l}\text { pitch diff, } \\
\text { window of } 3\end{array}$ & $\left\{{ }^{\prime}-12 \_-11 ',{ }^{\prime}-12{ }_{-}-11{ }_{-}-10^{\prime}, \ldots,{ }^{\prime} 11{ }_{-} 12^{\prime}\right\}$ & $\begin{array}{l}\{\text { 'Low', } \\
\text { 'High'\} }\end{array}$ \\
\hline Cat-pos-HL & $\begin{array}{l}\text { position in } \\
\text { trial set }\end{array}$ & $\left\{{ }^{\prime} \mathrm{A}, \ldots,{ }^{\prime} \mathrm{E}\right.$ ' $\}$ (stimuli 1-5 within trial set) & $\begin{array}{l}\{\text { 'Low', } \\
\text { 'High'\} }\end{array}$ \\
\hline Cat-val-SD & $\begin{array}{l}\text { pitch value, } \\
\text { window of } 3\end{array}$ & $\left\{{ }^{\prime} 1 \_2,{ }^{\prime} 1{ }^{\prime} 2 \_3,, \ldots,{ }^{\prime} 12 \_13 '\right\}$ & $\begin{array}{l}\{\text { 'Std', } \\
\text { 'Dev'\} }\end{array}$ \\
\hline Cat-diff-SD & $\begin{array}{l}\text { pitch diff, } \\
\text { window of } 3\end{array}$ & 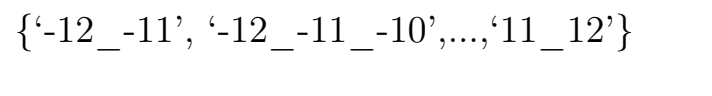 & $\begin{array}{l}\{\text { 'Std', } \\
\text { 'Dev'\} }\end{array}$ \\
\hline Cat-pos-SD & $\begin{array}{l}\text { position in } \\
\text { trial set }\end{array}$ & $\left\{{ }^{\prime} \mathrm{A} ', \ldots,{ }^{\prime} \mathrm{E} '\right\}$ (stimuli 1-5 within trial set) & $\begin{array}{l}\{' S t d ', \\
\text { 'Dev'\} }\end{array}$ \\
\hline
\end{tabular}

Table 2. Prediction simulations. Overview of the different types EDL simulations in the predictive learning analysis. For predictive learning, the cue is always the stimulus prior to the outcome, except in the case of bigrams, where the cues are a series of the three bigrams before the outcome. Note that in the case of bigrams, in order to keep the table size to a minimum, example bigram cues are provided, rather than the full set.

\begin{tabular}{|c|c|c|c|c|}
\hline Simulation & Cue type & Possible input cues & \multicolumn{2}{|c|}{ Outcomes } \\
\hline Pred-val-HL & $\begin{array}{l}\text { pitch value, } \\
\text { window of } 3\end{array}$ & $\left\{{ }^{\prime} 1 \_2^{\prime},{ }^{\prime} 1 \_2 \_3 ', \ldots,{ }^{\prime} 12 \_13 ’\right\}$ & \multirow{3}{*}{\multicolumn{2}{|c|}{$\begin{array}{l}\text { \{'Low', } \\
\text { 'High'\} } \\
\text { \{'Low', } \\
\text { 'High'\} } \\
\text { \{'Low', } \\
\text { 'High'\} }\end{array}$}} \\
\hline Pred-diff-HL & $\begin{array}{l}\text { pitch diff, } \\
\text { window of } 3\end{array}$ & $\left\{{ }^{\prime}-12_{-}-11\right.$ ', ' $\left.-122_{-}-11_{-}-10^{\prime}, \ldots,{ }^{\prime} 11_{-} 12^{\prime}\right\}$ & & \\
\hline Pred-bg-HL & tone bigrams & (e.g. 'HH_HH_HH', 'HH_HH_HL') & & \\
\hline Pred-val-SD & $\begin{array}{l}\text { pitch value, } \\
\text { window of } 3\end{array}$ & $\left\{{ }^{\prime} 1 \_2 ',{ }^{\prime} 1 \_2 \_3 ', \ldots,{ }^{\prime} 12 \_13^{\prime}\right\}$ & \multirow{3}{*}{\multicolumn{2}{|c|}{$\begin{array}{l}\text { \{'Std', } \\
\text { 'Dev'\} } \\
\text { \{'Std', } \\
\text { 'Dev'\} } \\
\text { \{'Std', } \\
\text { 'Dev'\} }\end{array}$}} \\
\hline Pred-diff-SD & $\begin{array}{l}\text { pitch diff, } \\
\text { window of } 3\end{array}$ & 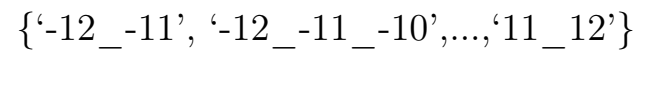 & & \\
\hline Pred-bg-SD & tone bigrams & (e.g. 'HH_HH_HH', 'HH_HH_HL') & & \\
\hline Pred-val-val & $\begin{array}{l}\text { pitch value, } \\
\text { window of } 3\end{array}$ & $\left\{{ }^{\prime} 1 \_2^{\prime},{ }^{\prime} 1{ }^{2}{ }^{2} 3^{\prime}, \ldots,{ }^{\prime} 12 \_13^{\prime}\right\}$ & $\begin{array}{l}\{\text { Pitch } \\
8-13\}\end{array}$ & $1-6$, \\
\hline Pred-diff-val & $\begin{array}{l}\text { pitch diff, } \\
\text { window of } 3\end{array}$ & $\left\{{ }^{\prime}-12 \_-11,{ }^{\prime}-12--11_{-}-10^{\prime}, \ldots,{ }^{\prime} 11_{-} 12^{\prime}\right\}$ & $\begin{array}{l}\{\text { Pitch } \\
8-13\}\end{array}$ & $1-6$ \\
\hline Pred-bg-val & tone bigrams & (e.g. 'HH_HH_HH', 'HH_HH_HL') & $\begin{array}{l}\{\text { Pitch } \\
8-13\}\end{array}$ & $1-6$, \\
\hline
\end{tabular}


In the categorisation simulations (Table 1), cues that are part of the outcome stimulus are used to categorise the outcome stimulus into different categories. Specifically, in six separate simulations, one of the cue types pitch value, pitch difference or position was used to categorise the outcome stimulus into either high vs. low or standard vs. deviant. In the prediction simulations (Table 2) incoming cues were used to predict the outcome in the upcoming trial. Specifically, in nine separate simulations, one of the cue types pitch value, pitch difference or tone bigram was used to predict one of three different outcome types, namely high vs. low tone, standard vs deviant or pitch value. Note that we do not expect the pitch value simulations to perform well, for the reasons explained above; namely, that the pitch differences of the continuum steps (even two steps apart) were usually too small to discriminate (Nixon et al., 2018). However, it is possible that even if participants did not discriminate these tokens in the behavioural data, the ERP response could still show evidence of prediction of the pitch value. The pitch value simulations test this possibility.

\subsection{An illustrative example: Model predictions for Pred-bg-HL}

To illustrate how our simulations predict participants' expectations on each trial, Figure 5 visualises the activations for all experimental stimuli in the experiment generated by the Pred-bg-HL model for one participant. The trials are colour coded according to the position of the sound in a trial set, ranging from 1-5. (The colour coding is for visualisation purposes only; the simulation input does not include the trial set number.) This EDL model uses a sequence of bigram cues to predict whether the next stimulus will be a high or low tone. The unique cues and outcomes for this EDL model are presented in Table 3. What stands out from the activation pattern is that this EDL model generally has a stronger expectation for a standard stimulus to appear (i.e., stimuli 1-4) than a deviant (i.e., stimulus 5), with the activations for stimulus 5 considerably lower than for the other stimuli. Note that this occurs without the model having explicit information about which stimuli are standards or deviants. The reason is that the cues for the deviant stimuli do not uniquely predict the deviant outcome, but also appear with the standard outcome (i.e., cues 'HH' in the odd blocks and 'LL' in the even blocks) ; see Table 3). Further, the activations show a consistent change within each block. The activations of the standards are mainly determined by the cues 'HL' and 'LH', which predict the outcome High in the odd blocks and the outcome Low in the even blocks (see Table 3 below). Therefore, the learner needs to change their expectations based on these cues in every new block. The activations of the deviants, on the other hand, are determined only by the cues 'HH' in the odd blocks and 'LL' in the even blocks. Only one of these cue types occurs within each block. These cues occur more often with high tone outcomes in the odd block and low tone outcomes in the even block. Therefore, the expectation of each outcome is determined by cue competition. Note that outcome frequency also plays a key role, but the expectations

Figure 5. Activations (y-axis) for all stimuli of one experimental session (i.e., the trial order presented to one of the participants), generated by EDL model Pred-bg-HL. The legend shows the colour coding for the 5-trial sequence. In the odd blocks, stimuli 1-4 are high tones and stimulus 5 is a low tone; in even blocks stimuli 1-4 are low tones and stimulus 5 is a high tone. The cues are bigram tone sequences and the outcomes the tone of the next stimulus (e.g., ' $\mathrm{HH}_{-} \mathrm{HH}_{-} \mathrm{HL}^{\prime}$ are the cues predicting stimulus 5). The activations represent the strength of expectation for this outcome to appear. The x-axis shows the order of incoming stimuli (5 pitch sounds per trial set $x 42$ trial sets per block $=210$ stimuli per block $x 8$ blocks). The vertical dashed lines separate the blocks. 
are learned through a discriminative process of prediction and prediction error, not direct frequency counts.

Thus, this EDL model predicts a difference in expectation between standards and deviants, and a repeating fluctuation in expectations that is associated with the switching of standards and deviants between the odd and even blocks. This fluctuation in expectations is not expected for generative models of statistical learning, because it is only predicted when unlearning of associations plays a role (which is not the case in models based on co-occurrence probabilities).

\begin{tabular}{l|ll|l} 
Stimuli & Cues & Outcomes & Blocks \\
\hline 1 & 'HH_HH_HL_x' & High & \\
2 & 'HH_HL_LH_x' & High & \multirow{2}{*}{ 1, 3, 5, 7 (odd) } \\
3 & 'HL_LH_HH_x' & High & \\
4 & 'LH_HH_HH_x' & High & \\
5 & 'HH_HH_HH_x' & Low & \\
\hline 1 & 'LL_LL_LH_x' & Low & \\
2 & 'LL_LH_HL_x' & Low & \multirow{2}{*}{$2,4,6,8$ (even) } \\
3 & 'LH_HL_LL_x' & Low & \\
4 & 'LH_LL_LL_x' & Low & \\
5 & 'LL_LL_LL_x' & High &
\end{tabular}

Table 3. Cues and outcomes for the ED $\bar{D}$ simulation Pred-bg-HL. The ' $x$ ' cue is the 'background cue'. This is included in all trials in all simulations to capture the laboratory environment. The left column shows the position of the outcome stimulus (i.e. current trial) in the trial set. Outcomes were lexical tones, high or low. The cues consist of a sequence of trials implemented as overlapping tone bigrams (two trials of the lexical tone high $(\mathrm{H})$ or low $(\mathrm{L})$ ). The ' $\mathrm{x}$ ' cue is the 'background cue'. This is included in all trials in all simulations to capture the laboratory environment.

\section{Analysis: Temporal response modelling}

Analysis involved 1) generating trial-by-trial predictions of the ERP waveform using TRFs based on the EDL activations and 2) generating predictions of the ERP waveform for a set of control models for comparison. As outlined in Section 4, three tests were carried out to evaluate whether the trial-by-trial predictions of the ERP waveform derived from the EDL activation measure outperformed those derived from the control models in predicting the trial-by-trial EEG data. Below, we first explain how the trialby-trial estimates of the ERP waveform are obtained for the EDL-derived models using Temporal Response Function (TRF) fitting. We then explain how each of the control models was generated and how they were used to test the EDL models.

\subsection{Using trial-by-trial estimates to predict the ERP waveform}

The EDL simulations give us an estimate of how expected the incoming stimulus is on each trial (i.e., the activation of the outcome). The next step is to fit TRFs, which give us a prediction of the ERP waveform based on the input signal provided by the EDL estimates per trial.

We use the MNE toolkit for Python (Gramfort et al., 2013) to fit TRFs for our dataset. For each EDL simulation, there is an input signal consisting of activation values from the EDL simulations, i.e., a unique series of activation values for each participant, one value per incoming sound. TRFs are fitted for each EDL model, for 
each electrode, for each participant, and for the two time windows. We evaluate the fit of the TRFs for each EDL simulation, time window and electrode, hence aggregating over participants.

The TRFs are fitted so that the predicted response signal (the convolution of the input signal with the TRF) has an optimal fit to the ERP signal. Crucially, the input signal is not fitted, and hence the extent to which the TRF captures the ERP output signal is a measure of the relation between input and output.

\subsubsection{Teasing apart the effect of expectation from the overall auditory response}

Care was taken to avoid spurious fits (overfitting) and fitting to other properties of the signal. Because the EEG signal is highly variable, with many contributions to the variation, most of the EEG signal will not be predicted by any convoluted input. On the other hand, the mere occurrence of a stimulus likely leads to a predictable EEG response which will be captured by the TRFs. If the modelling approach does not account for a neural response to the onset of incoming stimuli in general, the TRF approach may be vulnerable to spurious fits. For analysis purposes, we can conceptualise this situation as a) an ERP component that occurs in response to any incoming stimulus and b) a modulation to that component depending on the level of expectation. We are interested in modelling the modulation, but because it is combined with the ERP response to the incoming stimulus, we need to tease them apart.

To tease apart the confound between the ERP component and the modulation of the component, two TRF shapes are generated using two-dimensional spikes. One dimension of the spike train is used to capture the brain's response to the onset of an (any) auditory stimulus. In this dimension, all spikes are of equal magnitude; thus, the dimension simply indicates stimulus onset and does not capture any variable properties of the stimulus. We call this the constant dimension. A second dimension is used to capture variation in the brain response, depending on how expected a stimulus is on a given trial. We call this the variable dimension. The use of two dimensions also allows us to avoid a potential technical difficulty. Because the ERP components measure error or surprise (low expectation), a priori we expect a larger (negative) ERP amplitude as activation/expectation increases. This inverse relationship would not be possible to model with a single dimension. ${ }^{4}$ Using two dimensions enables us to use the constant dimensions to predict the ERP component that occurs in response to unexpected stimuli, while the variable dimension can be used to predict how much smaller this component is as activation increases. Thus, larger spikes in the variable dimension can now be used to predict a decrease without generating an output signal that is overall positive.

Linking the two TRFs to our activation values, the TRF for the constant dimension represents minimum expectedness (activation of 0 ) and combining both TRFs (adding the TRF for the constant dimension together with the TRF for the variable dimension) represents maximum expectedness (activation of 1 ). The predicted response signal for all intermediate activation values is a linear interpolation between these two TRFs. Figure 6 illustrates the convolution: An idealised TRF is shown on the left (red line). A spike train of measures represents, per trial, the simulated activation of the presented

\footnotetext{
${ }^{4}$ Recall that the input vector consists of a valued spike (height determined by the activation value) as the initial value (stimulus onset). Thus, if the TRF used only a single dimension, then when the input signal contained small values of the initial spike (i.e. activation values close to zero), the TRF would predict a waveform that is similar to when there is no stimulus and therefore no input signal, i.e. the baseline signal. If this were the case, small values of activation would produce a TRF that predicts a small ERP in response to low activation. In other words, there would be no way to model a large ERP waveform in response to unexpected stimuli.
} 
TRF

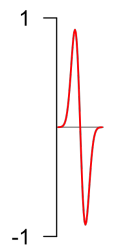

Time $\rightarrow$

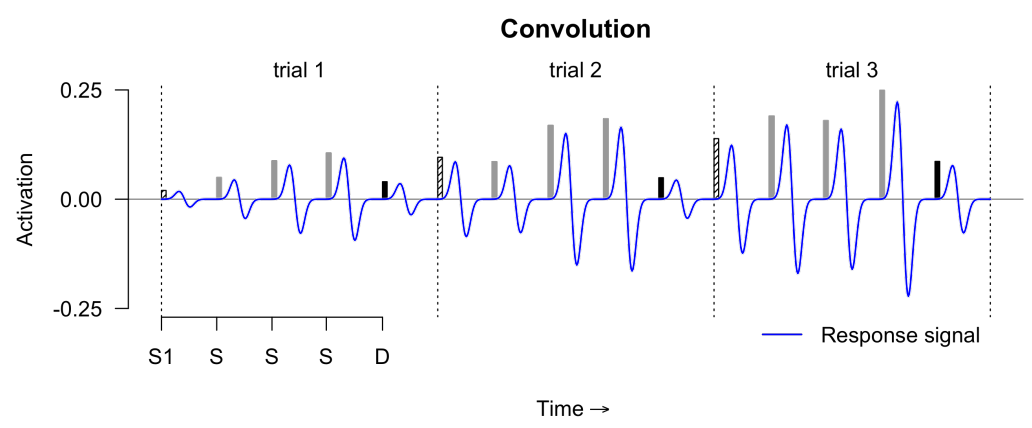

Figure 6. Temporal response function (TRF) as a convolution. Left: An idealized TRF (red line), representing a positive response shortly after a stimulus and a negative response later. Right: The predicted response signal (blue line) is the convolution of the TRF function and the activation spike (i.e. the input signal). The height of the spikes represents the estimated activation of the stimulus, derived from the EDL simulations. As the spikes have no duration, the convolution is simply the multiplication of the TRF with the height of the spike. Three experimental trial sets of five trials are shown; for illustrative purposes, the standards are shown in grey, the deviants in black, and the start of a trial set as a hatched spike. Fitting (not shown) entails generating multiple TRF shapes and selecting the TRF shape that is closest to the (EEG) signal.

stimulus (right; black or grey vertical lines). This spike train is convoluted with the TRF. The output is a continuous predicted response signal (blue line).

\subsubsection{Obtaining estimates for the timing and location of effects}

An advantage of TRF fitting is that, while our simulations predict the level of expectation on each trial, the neural correlate of the expectation - the ERP component does not need to be known in advance: it is derived from the data. Any relationship between the input signal (activation) and ERP data should be captured by the TRF. For example, if activation values are larger on trials that have a larger response at $200 \mathrm{~ms}$ and smaller on trials that have a smaller response at $200 \mathrm{~ms}$, the model will capture this with a TRF that increases with activation value at $200 \mathrm{~ms}$. The more the activation corresponds to the magnitude of the ERP signal (anywhere in the time window under analysis), the better the predicted response signal will fit to the observed ERP signal. If there is a linear relationship between the activation (spike magnitude) and the ERP component, there will be a good fit.

Thus, fitting TRFs not only allows us to detect whether there is a relationship between the trial-by-trial learning estimates and the ERP waveform, but also generates a prediction of the ERP waveform itself, for each trial in the dataset. Because separate TRFs are fitted for each electrode, the goodness-of-fit can be evaluated per electrode channel, revealing the location of the predicted effect. Although estimates of the ERP waveform are generated in a continuous manner anywhere in the window under analysis, the statistical test of significance is performed for the TRF as a whole. Therefore, given that either early or late ERP components or both may be involved in prediction and learning as outlined in Section 2.2, the present study separately fits TRFs in an early and a late window, 0-350 ms and 250-600 ms after stimulus onset, respectively. By separating the data into two time windows we can determine with more precision when the predicted effect actually fits the signal. 


\subsubsection{TRF fitting procedure}

TRFs are fitted using five-fold cross-validation. All data gathered from one participant at one electrode channel, in one of the two time windows is split into five parts. Using four parts as training data, TRFs are fitted to maximize $R^{2}$. The fitted TRF is then used to generate prediction for the remaining data, the test set, providing log-likelihoods for the test sets - which, over the five iterations of the cross-validation, comprise all the data. We then calculate the AIC of the fitted TRFs, and compare AIC values to assess whether the EDL-based TRF is more informative than a TRF fitted on a baseline model and a model based on probability, as in Ahmed (2021). The AIC/likelihood and $R^{2}$ scores are thus calculated only on the test sets (i.e., data not used for fitting). Note that all TRFs shown below are average TRFs over five iterations and over all participants.

\subsection{Evaluation: Testing the EDL models against control models}

Section 4 introduced three tests, of which the first two are against the baseline and probability models and the third is against a set of random models. Below, after a note about correcting for multiple comparisons, we provide more detail about how each of these models was created and how the tests were carried out. At the end of this process, we are left with a set of informative EDL models, which indicate what kind of learning predicts neural patterns in the ERPs; and a set of electrodes and time windows that indicate where and when the components related to trial-by-trial learning occur.

\subsubsection{Multiple comparisons}

As described in Section 5, the present study examined several possible EDL simulations. Note that, to avoid computational complexity, each different EDL simulation is used to generate a separate TRF model, rather than including multiple EDL simulations as input dimensions for one model. However, the EDL simulations are not considered to be independent, because the input for all the different models consists of spikes at certain time points (at the onset of the spike train, i.e. at stimulus onset) and zeros at all other time points. Therefore, because of the zeros, the input is highly correlated between models. In addition, there could potentially also be similarity between EDL simulations in the size of the onset spike itself. Further, we assume that different EDL simulations may capture different aspects of learning that are not mutually exclusive, but may take place in parallel. We therefore refrain from formally comparing different models based on different EDL simulations.

Because the statistical analyses test whether any EDL simulation can predict part of the ERP signal, they require corrections for multiple comparisons. In this respect, theoretically, all EDL simulations could turn out to represent exactly the same underlying construct; as such, the comparison of a control model with an EDL model may be a repeated test of the same hypothesis. Therefore significance is adjusted for multiple comparisons using Bonferroni corrections. Hence, $\alpha$ is divided by the product of the number of electrodes, time windows, and models (i.e., by 1280), to $\alpha=0.000039 .^{5}$

\footnotetext{
${ }^{5}$ In a previous version of this article, we considered 20 models, but in the interest of clarity and space, we have dropped five uninformative models. We still correct for the original number of models, instead of the 15 described above.
} 


\subsubsection{Baseline models}

On each trial, the input information that the EDL simulations use includes which type of outcome stimulus has occurred (e.g. high or low tone, standard or deviant). The EDL simulations learn to predict the outcome stimulus from the cues. The EDL predictions are measured in activation, which represents how expected the outcome stimulus is on each trial. However, if there are systematic differences between these stimulus types in the ERP response, it is possible that the EDL simulations might spuriously pick up on differences in the ERP response between these outcome stimuli. In this case, the EDL models might appear to perform well simply because different activation values correlate with differences in response to static properties of the stimuli, rather than because they capture trial-by-trial learning.

Two two-dimensional baseline models were constructed to capture only the fit that can be attained by separating the trials by outcome stimulus type and temporally aligning the TRF input signal with the stimuli. The EDL simulations were evaluated against these baseline models to assess whether the EDL predictions (activation) are more informative about the ERP signal than just the model input (the type of outcome stimulus). The High-Low (HL) baseline model serves a baseline for the EDL models that learned to categorise or predict the tone category (of which the name ends in '-HL') and the Standard-Deviant (SD) baseline model is for the EDL models that learned to categorise or predict standard vs. deviant stimuli (of which the name ends in '-SD'). Like the EDL models, each baseline model contains a constant dimension of uniformly sized spikes, which captures the occurrence of a stimulus (time-aligned to stimulus onset in the ERP data; see Section 6.1.1). In addition, the baseline models also contain a variable dimension to capture the difference between the stimulus types: This dimension contains a spike for each high tone (HL baseline model) or a spike for each deviant (SD baseline model).

\subsubsection{Probability model}

As described in the Method section, the probability model served two purposes. Firstly, it serves the comparison model for the pitch value simulations (the '-val' simulations in Table 2), since it is not meaningful to create a categorical baseline model like those described above for this type of EDL model. This is because there are multiple (12) continuum steps, but no expected ordered effect of the continuum step, such as a larger ERP response to higher pitch values, for example. So for the val models, instead of comparing to a model based on the outcomes per se, they are compared to the probability model, which is based on the probability of the pitch value outcomes. Secondly, the probability models are also used as an additional test for the other EDL model types, since the probability model represents a theoretical model of learning. So the probability models test whether the EDL models capture variability in the ERPs that is not captured by statistical learning. As explained in Section 3, the stimuli consisted of a bimodal distribution of pitch stimuli corresponding to two lexical tones. To capture this statistical distribution, the probability models use a TRF for each probability value (see Appendix E1).

\subsubsection{Test against Baseline and/or Probability model}

Informativeness - i.e., how well a TRF predicts the data - is calculated using Akaike's Information Criterion (AIC), considering the TRF together with the input signal as a model, and the actual recorded EEG signal as data. The lower the AIC, the more 
informative a TRF is. The AIC is low when the maximum likelihood of the data is high, but is penalised for each included parameter, because it is defined as two times the number of parameters $k$ minus two times the maximum log likelihood of a model $m: \operatorname{AIC}(m)=2 k-2 \ln L$. As $k$ is constant for all our models, the AIC scores are reduced if the $\log$-likelihood $\ln L$ is reduced. The total $\log$-likelihood is the sum of the log-likelihood for all (unseen) test data in the five-fold cross-validation procedure described above. If two models (such as an EDL model and its baseline) are fitted on the same data, and the EDL model has a lower AIC than the baseline model, Equation 1 calculates the probability of the null hypothesis that the alternative (baseline) model and not the EDL model minimizes information loss.

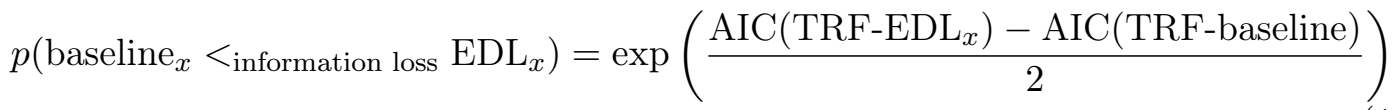

The p-values obtained in this way are used to evaluate significance, against $\alpha=0.05$, with corrections for multiple comparisons, i.e., for the number of TRFs, which is equal to the product of the number of EDL models, electrode channels, and time windows.

\subsubsection{Random models}

As an added sanity check, for the models that beat the baseline and probability models, we also test whether the trial-by-trial activation values from our EDL simulations perform better than a random sampling of these values. This test provides an additional check to ensure that it is very unlikely that the EDL models spuriously capture variation in the EEG data. These random models are based on the activation values sampled from the set of all EDL simulations. Because the activation values are sampled from the EDL activations, correlation with the ERP response is still possible - and expected - in the random models. Therefore, we do not expect the EDL models to always outperform the random models. However, if the EDL-derived models perform well, they should achieve a higher correlation with the ERP response than the random models do.

To produce the spike values in the variable dimension for each random model, we sampled one activation value per trial for each trial of the experiment (1680 trials) from the activation values of all the EDL simulations. This was repeated 5000 times to produce 5000 random models. We fit TRFs for these random signals and compared the resulting models to the EDL models. The comparison uses explained variance, calculated as $R^{2}$, for each channel at each time window. Since we cannot assume any specific distribution for the $R^{2}$ values, we perform a random comparison test: each EDL model is compared (at each time window and at each electrode separately) to all 5000 models. The outcome variable is the proportion of hits (beating a random model) against the total number of comparisons. Significance is calculated against the null hypothesis that the hits are as likely as the losses (a binomial test against 0.05), with the alpha level Bonferroni-corrected for the number of channels and time windows (64) to $p<0.00078$. Note that the correction does not take the number of models into account, as we have already tested whether there are models that are more informative than a baseline model. 


\section{Results}

Several EDL-derived TRF models passed all the relevant statistical tests in several channels. That is, they were significantly more informative than the baseline models (Test 1, for the 'HL' and 'SD' models), or the probability model (Test 2, for the 'val' models) and were likely to explain more variance than random models (Test 3). In addition, several of the 'HL' and 'SD' models also passed the extra test against the Probability model (Test 2). Hence, the information that the EDL simulations provide about trial-by-trial learning is predictive of the ERPs. Overall, the EDL-derived models were more often more informative than the baseline models for the early time window ERP than for the late time window. EDL-derived models also outperformed the probability model more often in the early time window. In contrast, only one model was more informative than the probability model in the late time window and in only a few channels. There was only one channel, FC5, in which none of the EDL models outperformed their baselines - and this was only the case in the late time window. This time window/channel combination was therefore pruned. Some EDL-derived models beat their baseline in a few channels in the late time window, but fewer than the early time window. Finally, two models never outperformed their baseline or the probability model: Cat-val-HL and Cat-pos-SDs these models are also pruned.

Figure 7 shows the model-channel-window combinations in which the EDL models outperformed the baseline model (i.e., Test 1 for the '-HL' and '-SD' simulations), or the probability model for the '-val' simulations (Test 2) and also outperformed the random models (Test 3).

Early time window: The prediction models Pred-bg-HL, Pred-val-HL, Pred-diffHL, and Pred-bg-VAL, Pred-val-VAL, Pred-diff-VAL show a broad and overlapping scalp distribution in the early time window. The distribution of the categorisation models Cat-diff-HL, Cat-pos-HL, Cat-val-SD and Cat-diff-SD each partially matches the electrodes of the prediction models. Together, the EDL models capture variance in the ERP signal over a wide scalp distribution in the early time window: frontolateral (F7, F8, FC5, FC6,T7, T8) and centroparietal/occipital electrodes (P3, P4, Pz,C3, $\mathrm{CP} 1, \mathrm{CP} 2, \mathrm{CP} 5, \mathrm{PO} 3, \mathrm{Oz}, \mathrm{O} 2)$. The categorisation models Cat-val-SD and Cat-diff-SD seem more localised, showing up frontolateral in case of Cat-val-SD, and central in case of Cat-diff-SD, though their absence in other regions is a null result and should not be over-interpreted as proof of absence. In the early time window, none of the EDL models outperformed random models in channels Fp1, AF3, F3, FC1, P7, O1, PO4, $\mathrm{P} 8, \mathrm{CP} 6, \mathrm{C} 4, \mathrm{FC} 2, \mathrm{~F} 4, \mathrm{AF} 4, \mathrm{Fp} 2, \mathrm{Fz}$, and $\mathrm{Cz}$, so we refrain from interpreting these combinations.

The results for the comparison against the probability model (Test 2) are shown in Appendix C1. In the early time window, in almost all channels in which an EDL model outperformed its baseline, it also outperformed the probability model.

Late time window: Compared to the early time window, the EDL models seem to capture less additional variance in the ERP signal in the late time window, compared to the control models. Mainly the HL models - the models that learned the lexical tone category (categorisation models) or predicted the upcoming lexical tone (prediction models) - were more informative than their baseline in the late time window (except for categorisation model Cat-val-SD which is also found to be informative in two channels). In the late window, channels in which the EDL models were found to be more informative than their baselines were concentrated in the centroparietal-occipital electrodes $\mathrm{Cz}, \mathrm{CP} 1, \mathrm{CP} 2, \mathrm{Pz}, \mathrm{P} 3, \mathrm{P} 4, \mathrm{PO} 3, \mathrm{PO} 4, \mathrm{O} 1, \mathrm{Oz}, \mathrm{O} 2$. In the late time window, none of the EDL models outperformed random models in channels F7, P7, P8, CP6, 
T8, FC6, F8, and Fp2.

Appendix $\mathrm{C} 1$ shows the comparison against the probability model (Test 2). Although several EDL models outperformed their baselines in the centroparietal-occipital electrodes (Figure 7), they rarely outperformed the probability model in the late window (Appendix C1). Only a few channels remain for Cat-diff-HL. This is in contrast to the early time window, in which the informative EDL models generally outperformed the probability model. This suggests that either statistical effects emerge later after stimulus onset and that therefore, EDL-derived models are unlikely to be more informative the probability model, or that EDL-derived models perform less well in the late time window generally.

Results are also shown in Table 4. Due to space limitations Table 4 only shows the models that beat the random models the most often in at least one channel. The results for all models are presented in Appendix B1. The numbers shown in Table 4 represent the proportion of random models outperformed by each EDL model. The scores were included in the table if the EDL models outperformed more than $50 \%$ of the random models in that channel and time window; if not, the cell is left empty. As these proportions of wins are always measured against the same 5000 random models, higher proportions indicate numerically better performance; proportions are in that sense comparable. However, proportion differences between models were not tested statistically. Also note that the proportion of wins only measures how many of the random models were beaten by the EDL model, not how much better the EDL model was than the random models. That is, the proportion measure does not take mean $R^{2}$ scores into account. Shown in bold are the models that beat the random model most often for each channel and time window for each learning task: categorisation or prediction.

\subsection{Interpretation of the EDL simulations}

The finding that some of the EDL models are more informative than their baselines demonstrates that the EDL simulations captured variation in the signal resulting from learning or prediction error, rather than merely from basic auditory processing of the stimulus and rather than only to static properties of the stimuli. In addition, several EDL models also outperformed the probability model, especially in the early time window, indicating that these EDL models are also more informative about the ERP signal than a model based on stimulus probability.

There is a substantial amount of overlap in the scalp distributions for the different EDL models, as shown in Figure 7. It is possible that this overlap indicates that these models (partly) capture the same patterns in the ERP signal. However, this is not necessarily the case: the ERP signal is rich and variable, and the EDL models may capture different aspects of the signal even within the same channel. To investigate whether these overlapping scalp distributions were due to overlap in EDL activation patterns, we analysed the similarity in activation patterns of the EDL models. Figure 8 shows the correlation between the EDL activation patterns based on the stimuli presented to a single participant. Note that although the activation values were calculated for each participant individually, the correlations between activation values from various EDL models are nearly identical between all participants (only for the 'val' models, small differences are to be expected; note that the fact that the EDL input is nearly identical does not mean the fitted TRFs have to be). The correlation matrix shows two groups of EDL models with similar activation patterns. The first group are the models that 
Table 4. Proportion of random models beaten for selected* EDL models, for channel/time windows combinations for which at least one EDL model beat its baseline model. Bonferroni corrected alpha level for 64 (32 channels $\times 2$ time windows) comparisons, values only shown if $p<0.00078125$. Boldface text indicates the best score for the electrode channel for each task, categorisation (Cat) or prediction (Pred). Italics indicate that a particular model beat its baseline model for that channel and time window. ${ }^{*}$ Due to space limitations, this table presents only EDL models which beat the random models the most times in at least one channel and time window. For the full list, see Appendix B1.

Time window $0-350 \mathrm{~ms}$

\begin{tabular}{|c|c|c|c|c|c|c|c|}
\hline \multirow[b]{3}{*}{ Channel } & \multicolumn{7}{|c|}{ Model } \\
\hline & \multicolumn{3}{|c|}{ Cat. Models } & \multicolumn{4}{|c|}{ Pred. Models } \\
\hline & diff-HL & diff-SD & val-SD & bg-HL & diff-HL & diff-SD & val-HL \\
\hline F7 & 0.55 & 0.67 & 0.88 & 0.88 & 0.79 & 0.56 & 0.78 \\
\hline FC5 & 0.61 & 0.62 & 0.89 & 0.87 & 0.78 & 0.61 & 0.83 \\
\hline $\mathrm{T} 7$ & 0.51 & 0.63 & 0.69 & 0.68 & 0.64 & 0.59 & 0.64 \\
\hline C3 & 0.61 & 0.79 & 0.83 & 0.84 & 0.77 & 0.61 & 0.82 \\
\hline CP1 & 0.55 & 0.68 & 0.75 & 0.75 & 0.74 & 0.59 & 0.73 \\
\hline CP5 & 0.52 & 0.72 & 0.70 & 0.70 & 0.67 & 0.55 & 0.64 \\
\hline P3 & 0.64 & 0.69 & 0.67 & 0.68 & 0.67 & 0.53 & 0.66 \\
\hline $\mathrm{Pz}$ & 0.65 & 0.62 & 0.65 & 0.64 & 0.66 & & 0.64 \\
\hline PO3 & 0.64 & 0.66 & 0.65 & 0.64 & 0.59 & & 0.60 \\
\hline $\mathrm{Oz}$ & 0.66 & 0.60 & 0.57 & 0.57 & & & 0.51 \\
\hline $\mathrm{O} 2$ & 0.65 & 0.62 & 0.63 & 0.60 & & & 0.55 \\
\hline $\mathrm{P} 4$ & 0.56 & 0.67 & 0.67 & 0.72 & 0.74 & 0.55 & 0.76 \\
\hline $\mathrm{CP} 2$ & 0.59 & 0.74 & 0.70 & 0.74 & 0.78 & 0.56 & 0.77 \\
\hline $\mathrm{T} 8$ & 0.57 & 0.68 & 0.81 & 0.86 & 0.81 & 0.87 & 0.84 \\
\hline FC6 & 0.62 & 0.82 & 0.94 & 0.92 & 0.89 & 0.76 & 0.89 \\
\hline F8 & 0.69 & 0.68 & 0.86 & 0.84 & 0.75 & 0.76 & 0.81 \\
\hline
\end{tabular}

Time window $250-600 \mathrm{~ms}$

\begin{tabular}{|c|c|c|c|c|c|c|c|}
\hline \multirow[b]{3}{*}{ Channel } & \multirow{2}{*}{\multicolumn{3}{|c|}{ Cat. Models }} & \multirow{2}{*}{\multicolumn{4}{|c|}{ Pred. Models }} \\
\hline & & & & & & & \\
\hline & diff-HL & diff-SD & val-SD & bg-HL & diff-HL & diff-SD & val-HL \\
\hline CP1 & 0.58 & & 0.59 & 0.59 & 0.51 & 0.64 & 0.53 \\
\hline P3 & 0.66 & 0.52 & 0.61 & 0.64 & 0.60 & 0.63 & 0.62 \\
\hline $\mathrm{Pz}$ & 0.72 & & 0.60 & 0.63 & 0.52 & 0.62 & 0.57 \\
\hline PO3 & 0.69 & 0.52 & 0.69 & 0.68 & 0.55 & 0.62 & 0.60 \\
\hline O1 & 0.69 & 0.56 & 0.73 & 0.73 & 0.59 & 0.62 & 0.62 \\
\hline $\mathrm{Oz}$ & 0.68 & & 0.63 & 0.62 & 0.54 & & 0.55 \\
\hline $\mathrm{O} 2$ & 0.67 & 0.57 & 0.66 & 0.62 & 0.55 & & 0.54 \\
\hline PO4 & 0.66 & 0.60 & 0.66 & 0.65 & 0.63 & 0.59 & 0.62 \\
\hline P4 & 0.59 & 0.56 & 0.66 & 0.72 & 0.67 & 0.65 & 0.67 \\
\hline $\mathrm{CP} 2$ & 0.57 & 0.55 & 0.59 & 0.66 & 0.62 & 0.65 & 0.61 \\
\hline $\mathrm{Cz}$ & 0.56 & 0.53 & 0.57 & 0.64 & 0.55 & 0.70 & 0.53 \\
\hline
\end{tabular}


Early time window

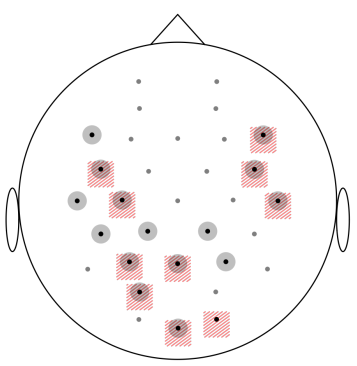

(1) Cat-diff-HL

Cat-pos-HL

b. Pred. Models:
(Illi! Cat-val-SD

$\square$ Cat-diff-SD

\section{Late time window}

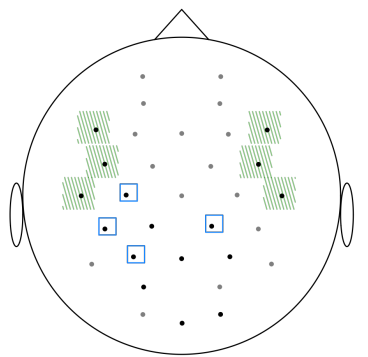

Cat-diff-HL

Cat-pos-HL

ilil' Cat-val-SD

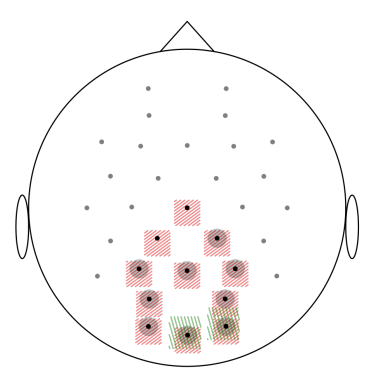

Late time window
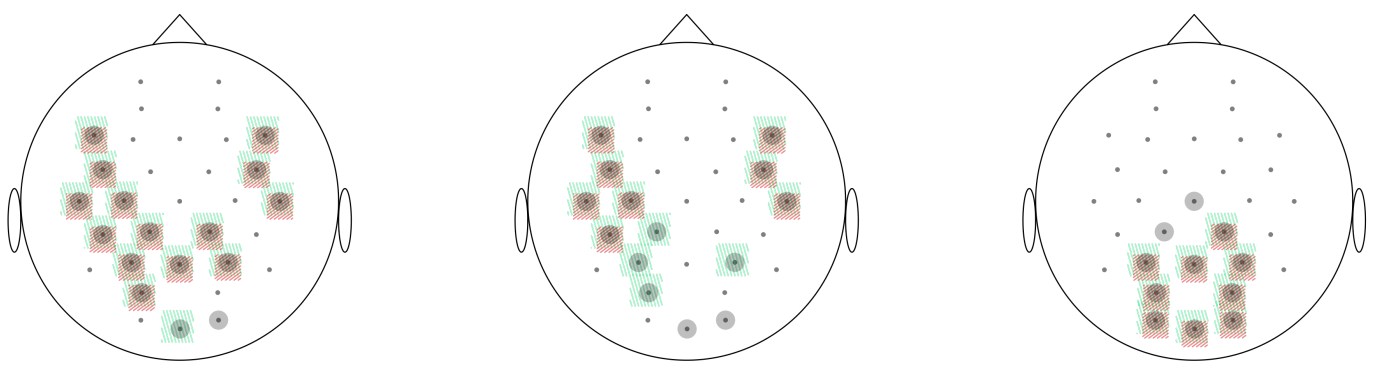

$\begin{array}{ll}\text { Pred-val-HL } & \text { Pred-val-VAL } \\ \text { Pred-diff-HL } & \text { Pred-diff-VAL } \\ \text { Pred-bg-HL } & \text { Pred-bg-VAL }\end{array}$

Pred-val-HL
Pred-diff-HL
Pred-bg-HL

Figure 7. Channel/time windows combinations for which EDL-derived models were more informative than their respective baseline models (for the models ending in -SD and -HL) or the probability model (for the models ending in -VAL), and were significantly more likely to be the model that explains more variance compared to a random model Top row a) shows the categorization models, bottom row b) shows the prediction models. 


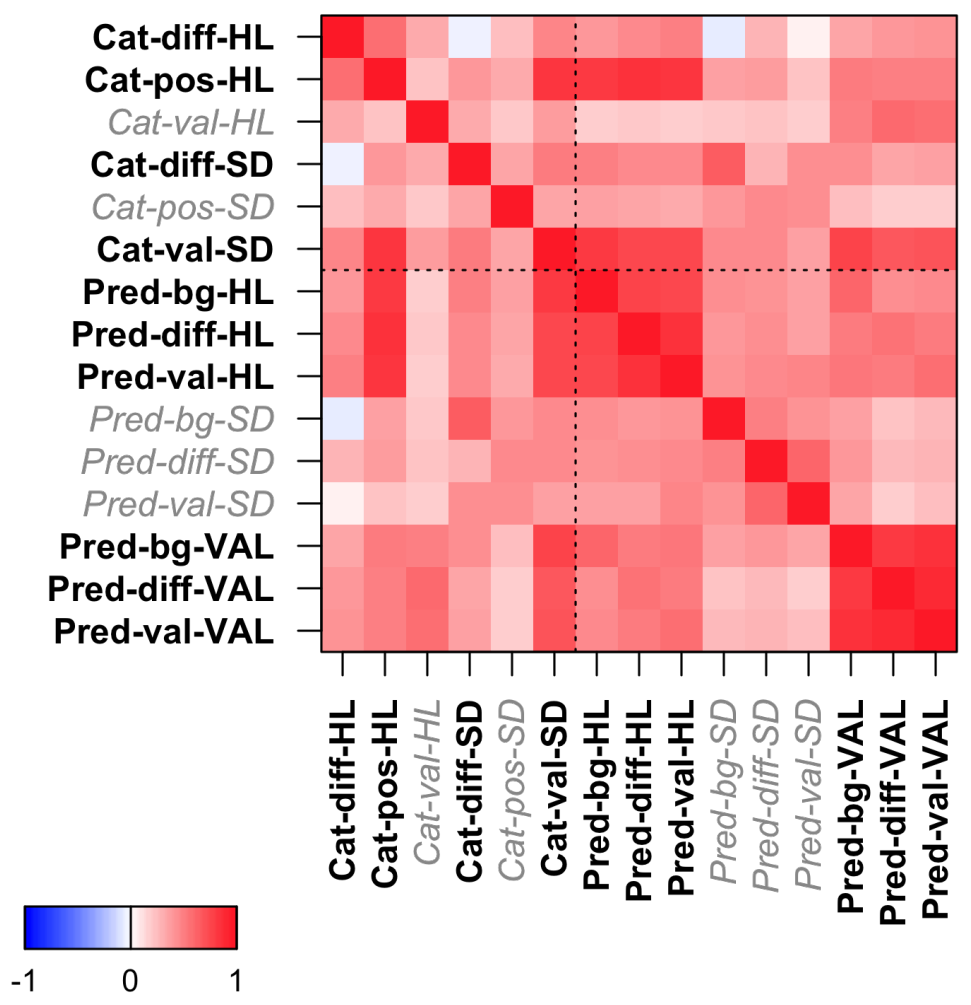

Figure 8. Matrix of Spearman's rho rank correlation coefficients representing the correlation between the activation values of the EDL simulations, ranging between -1 (blue) and 1 (red). The bold faced labels mark EDL models significantly more informative than their baseline (Test 1 or 2) and significantly more than $50 \%$ of the random models (Test 3).

predict the pitch value for the next stimulus, namely Pred-bg-VAL, Pred-diff-VAL, and Pred-val-VAL. Their correlations range between 0.83 and 0.91 . The activation values of these three models are included in Appendix D, Figure D3. It is interesting that the val models were informative about the ERPs. This result is somewhat surprising given that these models predict the specific pitch value, yet participants usually did not discriminate the pitch values in an overt behavioural task (Nixon et al., 2018). It is possible that the ERPs pick up on a level of processing that is more sensitive than the behavioural discrimination task.

The second group are the models that predict the tone (high vs low) of the next stimulus, namely Pred-bg-HL, Pred-diff-HL, and Pred-val-HL. Their correlations range between 0.78 and 0.86 . The activation values of these three models are included in Appendix D, Figure D1. In addition, two of the categorisation models - Cat-posHL and Cat-val-SD (Appendix D, Figure D2) - are strongly correlated with these prediction models. All these models have in common that they show lower activation values for the deviant stimulus than for the standards, and that the activation values of the standards show a strong increase over the course of each block. The model Cat-valSD shows a similar pattern as the group of prediction models, even though this model simulates the learning of categorising an incoming pitch value as standard or deviant, rather than learning to predict whether the next stimulus is a high or low tone. In the odd blocks, the lower pitch values are associated with the standard stimuli and the 
higher pitch values with the deviant stimuli. In the even blocks, the learner needs to switch this mapping and learn that the lower pitch values are now associated with the deviant stimuli and the higher pitch values with the standard stimuli. Thus, the pitch value cues and the standard vs deviant stimulus as learning outcomes captures the switching between blocks, which is also captured by the high vs low tone outcomes.

An important difference between this categorisation model and a prediction model such as Pred-bg-HL is that this Cat-val-SD model only categorises the current incoming stimulus, but cannot predict whether the next stimulus is going to be a standard or deviant. In other words, this model does not use the sequence of incoming sounds to predict the next stimulus - which we consider a highly plausible cognitive task in this experiment. This difference is visible in the trajectory of activation of the deviant stimulus over the course of the block, which increases for the categorisation model, but shows a ' $u$-shape' (decreasing-increasing) for the prediction models as a result of cue competition. This may be why the Pred-bg-HL model was informative over a larger number of electrodes and time windows than Cat-val-SD. However, our approach does not directly compare the different EDL models and, as a result, we cannot draw conclusions about which of the EDL model is most informative and whether the difference in explained variance would be significant. We consider the model Pred-bg-HL the most plausible from this group of models, because it is the only model that uses the sequence of stimuli as cues.

Two other models are worth mentioning: Cat-diff-HL and Cat-diff-SD. These two models show a correlation close to zero (-0.05), indicating that their patterns are not similar. In addition, these models do not show strong correlations with the other significant models (at most 0.51). Appendix D, Figure D2 shows the activation values for these two EDL models. These two models capture different aspects of the learning in this task from the informative prediction models described before. Both EDL models use the difference in pitch values between the preceding and current stimulus as cues, capturing the transition between sequential stimuli. When the difference in pitch is large, the incoming stimulus is likely a deviant stimulus, or the start of a new sequence of stimuli. When the difference is small, the incoming stimulus is likely to be another standard stimulus. As such, the EDL simulations show different activation patterns for the first stimulus, the last stimulus, and the other standards (i.e., stimuli 2-4). The two models learn to expect different outcome stimuli. On the one hand, model Cat-diff- $H L$ learns to categorise high and low tones, and therefore also captures the switching of associations between the odd and even blocks: A large difference between two sequential pitch values (i.e., a low tone followed by a high tone, and vice versa) provides a clue to classify the incoming pitch value as a high or low tone, which is consistent over all blocks. On the other hand, a small difference between two sequential pitch values (i.e., two low tones with slightly different pitch values or two high tones with slightly different pitch values) does not provide a consistent cue for classifying the incoming pitch value as high or low tone, because its association to the tone is switching between blocks. A prominent difference in comparison with the other models discussed in this section is that the activation values for the deviant tone (i.e., stimulus 5) are in the same range as the activation values of the other standard stimuli (i.e., stimuli 2-4). On the other hand, model Cat-diff-SD learns to categorise standard and deviant stimuli based on the same cues as the model Cat-diff-HL, resulting in the opposite effect in the activation pattern: A small difference between two sequential pitch values (i.e., two low tones with slightly different pitch values or two high tones with slightly different pitch values) provides a reliable clue to classify the incoming pitch value as a standard stimulus, which is consistent over all blocks. In contrast, a large difference between two 
sequential pitch values (i.e., a low tone followed by a high tone, and vice versa) does not provide a consistent cue for classifying the incoming pitch value as standard or deviant, because it differs per block whether a large negative difference is associated with a new sequence (i.e., the first standard) or with the deviant stimulus, and vice versa for a large positive difference.

Interestingly, the EDL models Pred-bg-HL, Pred-diff-HL, and Pred-val-HL are found to be informative in the same centroparietal electrodes as the models Cat-diff-HL and Cat-pos-HL in the later time window (see Figure 7), although they predict different activation patterns. This may be caused by these different models all capturing a similar aspect of the ERP response in these electrodes, such as the strong increase in activation for the standard stimuli 2-4, or the difference in activation between the first stimulus (i.e., stimulus 1) and last stimulus (stimulus 5, deviant). Alternative explanations may be that different participants show different ERP patterns that are captured by these different EDL models, or that the different EDL models capture variation at different times within the late time window. To gain more insight into how the EDL models are related to the ERP data, we will inspect the fitted TRFs.

\subsection{Visualising the TRFs: Relation between EDL simulations and ERP waveforms}

Figure 9 illustrates the TRFs that relate the activation patterns of EDL model Predbg-HL to the ERP measured in channel FC6 and Pz. Figure $9 a$ (top left) shows the TRF for FC6 in the early time window between $0-350 \mathrm{~ms}$ after stimulus onset and Figure $9 b$ (top right) shows FC6 in the late time window $250-600$ ms after stimulus onset. The black solid line shows the estimated signal fitted by the constant dimension, which contains a uniformly sized spike for every incoming stimulus. The thick red line shows the estimated signal fitted by the variable dimension, which contains for each stimulus a variable sized spike. The size of the spike is determined by the activation value generated by the EDL model Pred-bg-HL on each trial. The scalp plots are shown for the early time window (top) and late time window (bottom).

As explained in Section 6.1.1, the constant dimension represents the estimated ERP waveform for stimuli with an activation value of 0.0 and the variable dimension represents the additive effect for different activation values: For stimuli with an activation value of 1.0, the ERP signal is represented by the sum of the constant and variable dimension (the dotted line in Figure 9). Thus, the variable dimension captures the difference between ERPs elicited by an activation of 1.0 and an activation of 0.0 . For all intermediate values of activation, the variable dimension is scaled with the activation value to create a linear interpolation between the constant and variable dimension. Thus, an important assumption of this approach is that the differences in ERPs are linearly related to the differences in activation values.

In channel FC6, the difference in ERP amplitude as activation value increases is predicted to peak around $150 \mathrm{~ms}$ after stimulus onset (indicated by the yellow-marked time window in Figure $9 a$ ). The scalp plot shows the distribution of the differences in this time window over the scalp. Figure $9 b$ shows the TRF for the same model and channel in the late time window ranging 250-600 ms after stimulus onset. The modulating effect of the activation values on the estimated ERP signal is much smaller in the late compared to the early time window. This is not surprising, because model Pred-bg-HL did not significantly outperform the baseline models in channel FC6 in the late time window (see Figure 7). Figure 9 also shows the TRFs for model Pred-bg-HL 


\section{TRFs EDL model Pred-bg-HL}

a. Early time window FC6

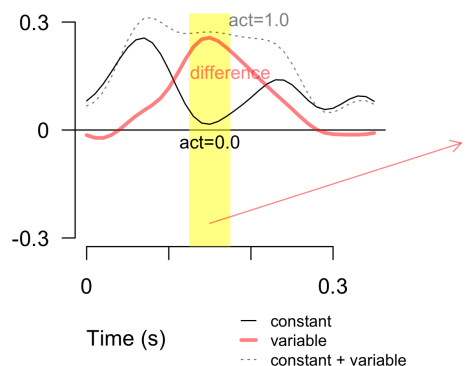

c. Early time window $\mathrm{Pz}$

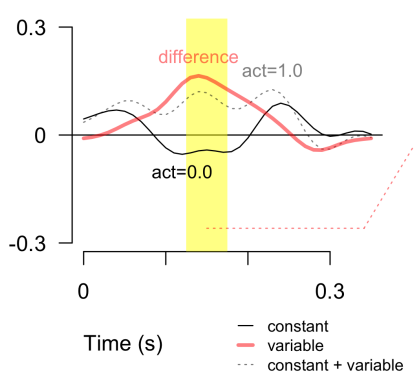

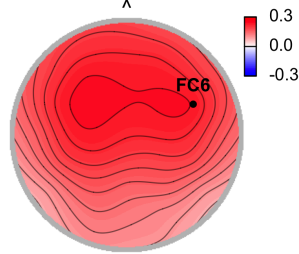

difference $0.125-0.175 s$ b. Late time window FC6

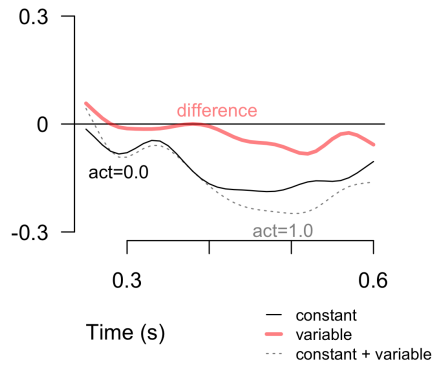

d. Late time window $\mathrm{Pz}$

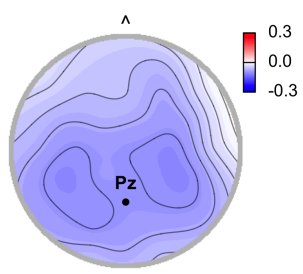

difference $0.500-0.550 \mathrm{~s}$

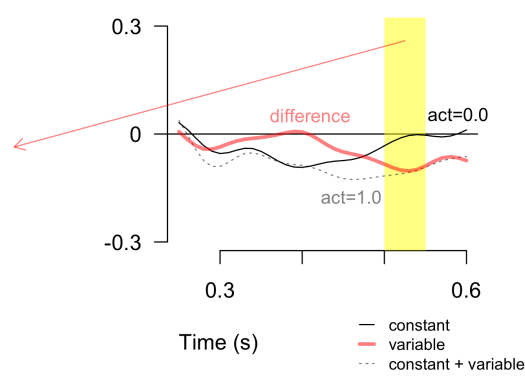

Figure 9. TRFs fitting the activations of EDL model Pred-bg-HL to the ERPs in channels FC6 (top row) and $\mathrm{Pz}$ (bottom row). As the TRFs are fitted on z-scores of the ERP data, the TRF scale has arbitrary units. The black solid lines show the constant dimension (representing an activation value 0.0), the red thick lines show the variable dimension (representing difference between the activation values 1.0 and 0.0 ), and the dotted lines show the sum of the constant and variable dimension (representing the activation value 1.0).

Panels $a$ and $c$ : TRFs early time window. The difference is predicted to peak around $0.150 \mathrm{~s}$ after stimulus onset, indicated by the yellow time window. Panels $b$ and $d$ : TRFs late time window. The EDL model only beats its baseline in channel Pz, but not in FC6. The scalp plot in panel a shows the distribution of the difference in the early, yellow-marked time window. The scalp plot in panel $d$ shows the distribution of the difference in the later yellow-marked time window. 


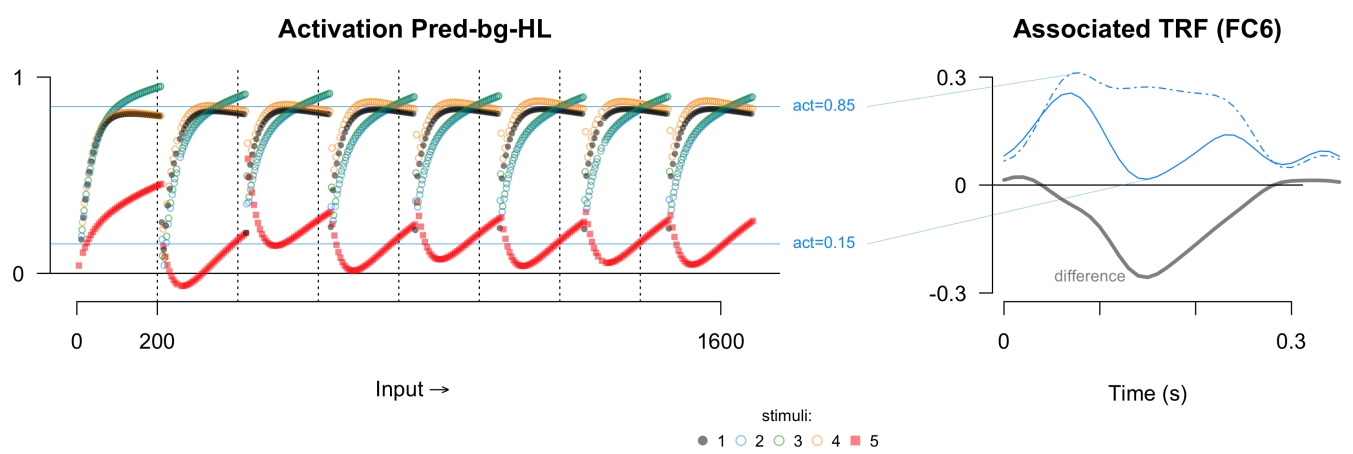

Figure 10. Relation between EDL activation values and the fitted TRF. Left: Activation pattern generated by model Pred-bg-HL ( Section 5.2), with each learning event on the x-axis and the activation on the y-axis. For interpretation, we indicated the activation values 0.15 and 0.85 with blue lines. Right: TRF fitting the activation pattern generated by model Pred-bg-HL to the ERP measured in channel FC6, with on the x-axis the time in seconds, and on the y-axis the TRF amplitude (arbitrary value). The blue lines represent the estimated signals for activation values of 0.15 (solid line) and 0.85 (dash-dot line). The grey thick line shows the difference the two blue lines and equals $(0.15-0.85) *$ variable dimension (see Figure $9 a$ ).

related to channel $\mathrm{Pz}$, in which the model outperformed the baseline models in both the early (Figure 9c) and late time windows (Figure $9 d$ ). In the early time window, the timing of the peak in $\mathrm{Pz}$ is similar to the timing in FC6, around $150 \mathrm{~ms}$ after stimulus onset. In the late time window, the peak emerges around $450 \mathrm{~ms}$ and peaks around $525 \mathrm{~ms}$ after stimulus onset.

Figure 10 illustrates how the TRFs relate to the activation values in the EDL simulations. Each dot in the simulation figure (left) represents the activation on that trial; that is, the estimate of how expected the stimulus is (in this case, the expectation that the next stimulus will be a high or low tone) based on the cues (in this case, a series of bigram cues). The TRFs (right) provide an estimate of how the EDL activation values modulate the ERP signal. In EDL model Pred-bg-HL, the deviant stimulus (i.e., stimulus 5 in each sequence of sounds) generally has a low activation value (median: 0.15, interquartile range (IQR): 0.08-0.23), whereas the standard stimuli (i.e., stimuli 1-4) generally have higher activation values (median: 0.82, IQR: 0.73-0.85).

However, the relation between the stimulus characteristics and the activation values is different for each EDL model. The TRFs of all EDL models have in common that the constant dimension always implements the response to the occurrence of a sound, and the variable dimension implements the expectation (activation) of the incoming sound. We can compare the TRFs of the EDL models by comparing the variable dimension, as this dimension estimates how the activation modulates the ERP signal. Figure $11 a$ compares the signals fitted by the variable dimensions of the most informative EDL models. Interestingly, the models with correlated activation patterns (i.e., Pred-bg-HL, Pred-val-HL, Pred-diff-HL, Cat-pos-HL and Cat-val-SD, see Figure 8) show a similar TRF, starting around $50 \mathrm{~ms}$ and peaking around $150 \mathrm{~ms}$ after stimulus onset. However, the TRF of EDL model Cat-diff-HL (red dotted line) looks different, with a double peak between $75 \mathrm{~ms}$ and around $150 \mathrm{~ms}$ after stimulus onset.

To further investigate how to interpret this series of models, we examined how the models capture differences in the response to standards and deviants. Although not all simulations used standard-deviant information in the input representations, the oddball design - i.e. a series of four tones, followed by a change of tone on the fifth stimulus - was one of the most prominent aspects of the experiment design. The ERP 
a. Comparison TRFs EDL models

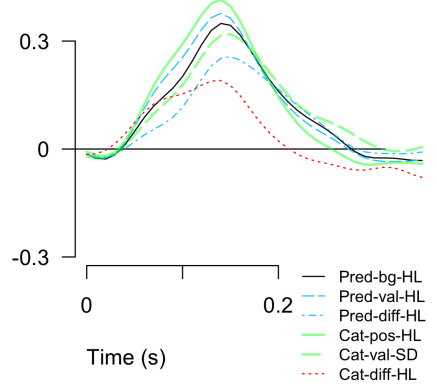

b. Median activations

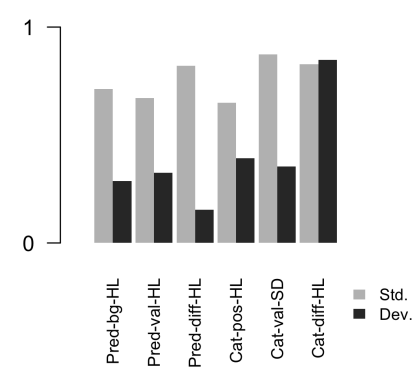

c. Comparison SD baseline

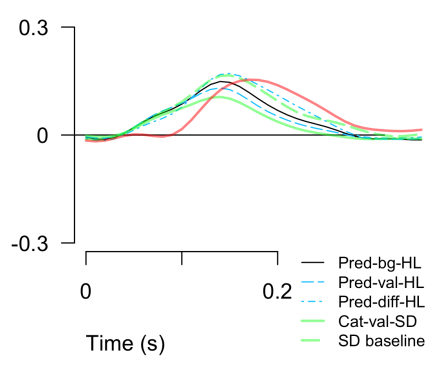

Figure 11. Evaluating TRF predictions. Panel a: Comparing the variable dimension of the TRFs for a group of EDL models with overlapping scalp distributions, namely Pred-bg-HL, Pred-valHL, Pred-diff-HL, Cat-valSD, and Cat-diff-HL. Panel b: The median activations for standard (grey bars) and deviant (black bars) stimuli, averaged over the complete experiment. Panel $c$ : Estimated difference in ERPs (i.e., scaled variable dimension) between trials with the same activation as the median of standards (see panel b) and trials with the same activation as the median of deviants (see panel b) for EDL models Pred-bg-HL, Pred-val-HL, Pred-diff-HL, and Cat-val-SD. In addition, the thick red line shows the estimated difference between standards and deviants, as modelled by the SD baseline (i.e., unscaled constant dimension of SD model).

data also show different responses to standards and deviants (Appendix A). Therefore, we expect that at least some of the models might capture a difference between standard and deviant stimuli.

Firstly, we calculated the median activation for standards and deviants for each of the models. For the models with correlated activation patterns, Figure $11 b$ shows higher activation values for the standards (range: 0.67-0.87) than for the deviants (range: 0.15-0.35). But for model Cat-diff-HL the median activation values for standards and deviants are the same ( 0.83 and 0.85 respectively). This means that, whereas the other EDL models have different activation values which predict a difference in ERPs between standards and deviants on average, model Cat-diff-HL does not predict any difference between standards and deviants (see also Appendix D, top left panel of Figure D2, for the trial-by-trial predictions for Cat-diff-HL).

Secondly, we investigated how the different EDL models predicted the ERP waveform and how these predicted ERP waveforms compare to a model that only used standards and deviants, namely the Standard-Deviant (SD) baseline model. Using the median activations in Figure 11b, we calculated the difference in median activations between standards and deviants for each EDL model. This difference in median activations was then used to produce a difference TRF that shows how the predicted ERP waveform changes between these activation values (the median activation value for standards and deviants, respectively). The difference TRF was calculated by scaling the variable TRF dimension by the difference in median activation. The difference TRFs are shown in Figure $11 c$ for the same EDL models as listed above. This difference TRF tells us two things. If the difference TRF of the EDL model is similar to the variable TRF of the SD baseline model, this suggests that the EDL model is able to capture differences between standards and deviants. On the other hand, any differences compared to the SD baseline model suggest that the EDL model is capturing additional effects on top of the difference between standards and deviants. Therefore, Figure $11 c$ also shows the (unscaled) variable TRF dimension for the SD baseline for comparison, which captures the estimated difference in ERP between standards and deviants (thick red line). Note that the estimate for model Cat-diff-HL is excluded, 
as this would be a straight line around 0 , because the difference in median activation, which scales the variable TRF dimension, is 0.02 .

Interestingly, the TRF of the SD baseline model estimates that the difference between standards and deviants starts around $100 \mathrm{~ms}$ after stimulus onset, but the TRFs of the EDL models suggest that the difference starts earlier, around 50ms after stimulus onset. These results show that the EDL models do capture a different response between standard and deviant stimuli, but do not only capture the difference between standards and deviants. Instead, they capture how expected a stimulus is. On some trials, this will correlate with the difference between standards and deviants, but this is not always the case. The expectation of what will follow from the input cues changes over the course of the experiment, especially within the blocks. So both standards and deviants have both high- and low-activation trials (see Figure D1).

Note that this effect of changing expectations over the course of each block is also captured by EDL model Cat-diff-HL. This model does not capture a difference between standards and deviants, but does show an early difference between high and low activations in the estimated ERP signals in Figure 11a. What is interesting is that, since Cat-diff-HL does not pick up on differences between standards and deviants, this Catdiff-HL model allows us tease apart a certain neural response pattern from that of the SD baseline model. When separated out, this pattern of results suggests that the EDL models are capturing two responses: an earlier response $(50-200 \mathrm{~ms}$ after stimulus onset, as captured by EDL model Cat-diff-HL) and a later response $(100-300 \mathrm{~ms}$ after stimulus onset, as captured by the SD baseline model). The EDL models Pred-bg-HL, Pred-val-HL, Pred-diff-HL, and Cat-val-SD capture both effects with their activation values. Taken together, we argue that the strength of participants' expectations about the outcome stimulus is what modulates the ERP signal, which changes trial-by-trial, not just between standards and deviants.

Appendix E provides a more extensive overview of the TRFs of the HL and SD baseline models, the probability model and the EDL models (Figures E5, E6 and E8). Based on the inspection of the TRFs we conclude that the EDL models that are found to be informative in the same centroparietal electrodes in the early time window (Pred-bg-HL, Pred-val-HL, Pred-diff-HL, Cat-val-SD, and Cat-diff-HL) partly capture the same patterns in the ERP data (stimuli 2-4), but also show differences (stimuli 1 and 5). First, all EDL models that are found to be informative in multiple electrodes show a sharp increase in activations for stimuli 2-4 over the course of each block. The cues associated with these stimuli are informative for whether the standard stimulus is a high or low tone, but this switches every block and therefore the associations need to be updated at the start of each block. The EDL models explain the precise dynamics differently. Second, these EDL models also seem to capture different aspects of the ERP data. The EDL model Cat-diff-HL shows a qualitatively different TRF in comparison with the other EDL models, because it does not capture the difference between standards and deviants. The reason is that model Cat-diff-HL predicts the activation values for stimulus 5 in the same range as the the activation values for stimulus $2-4$, whereas the other models predict lower activation values for stimulus 5 than for stimuli $1-4$.

In the present experiment, we cannot say with certainty exactly what underlies these two different temporal patterns in the neural response. However, there are a number of possibilities that we believe might explain this pattern. Firstly, it might be that the early effect captured by the Cat-diff-HL model reflects differences in expectation, while the later effect, captured by the SD baseline model, reflects detection of large pitch differences (i.e. the switch between standard and deviant). The cluster of other 


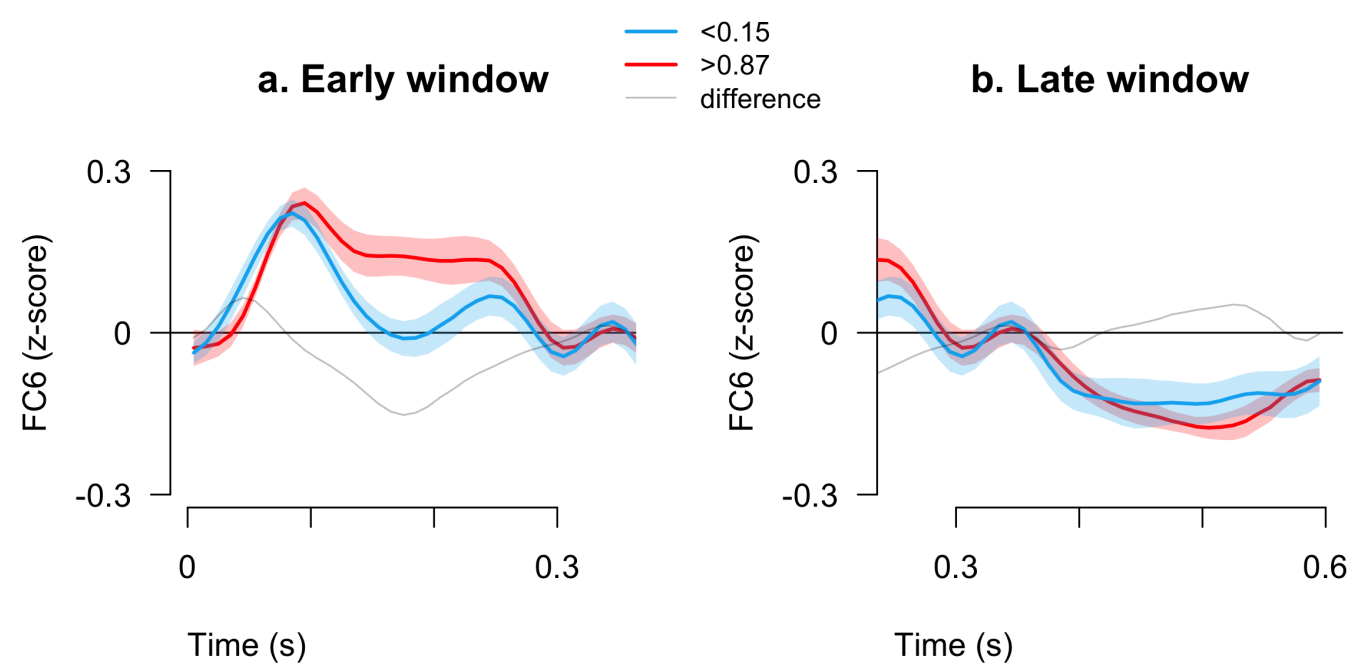

Figure 12. Grand averages in channel FC6 of the lowest $10 \%$ (blue lines) and highest $10 \%$ (red lines) of the activation values per participant with the error bars \pm 1 standard error of the participant means. The grey lines show the difference between the averages of the trials with the lowest and highest activation values.

informative models (i.e., Pred-bg-HL, Pred-val-HL, Pred-diff-HL, and Cat-val-SD) captures both of these effects. Another possibility is that the difference in timing reflects a change over the course of the block. In the Cat-diff-HL model, activation increases over the block for all stimuli, including the deviants. So it is possible that the early effect picks up on a more general development that, as participants become more familiar with the pattern in a particular block, the ERP responses occur earlier.

Further work is needed to tease apart these possibilities. However, it is worth noting that disentangling the timing of effects was made possible by having a model, Cat-diffHL, that was partly uncorrelated with the other models (see Figure 8). By generating a variety of EDL simulations and combining the EDL modelling with additional correlation analyses we were able to capture different parts of the ERP response signal. We see this as a fruitful path for future studies working with ERPs, which are a notoriously complex type of data.

As a final check we verified whether the patterns detected by the TRFs are also visible in the data. In order to do that, we added the activation values for EDL model Pred-bg-HL to the data. Note that for each individual experiment (i.e., a series of stimuli presented to an individual participant) a separate network was trained, so that for each unique participant-trial combination an activation value was calculated based on the cues and outcomes related to the presented stimulus and the current state of the network. To compare the TRFs to the data, we first calculated the $10 \%$ and $90 \%$ quantiles of the activation values per participant (mean values of 0.147 and 0.874 respectively). Based on these values, we selected the trials with activation values in the lowest 10\% $(n=2429)$ and the highest $10 \%(n=2429)$. Figure 12 shows the averages for the lowest (blue) and highest (red) quantiles and their difference (thin grey line). The patterns in the data are very similar to the TRF estimates in Figures $9 a$ and $b$. The TRF variable dimension, however, seems to peak slightly earlier $(0.150 \mathrm{~s}$ after stimulus onset) than the difference in the data (180 s after stimulus onset). Note that the TRF variable dimension estimates the difference between activation values 1.0 and 0.0 , whereas Figure 12 visualizes the difference between the activation values 
lower than 0.15 and higher than 0.87 . To summarise, the EDL simulations predict how expected the outcome stimulus is for the participants in the experiment, based on stimulus characteristics and the state of the learning network. The TRFs are used to link these activation values to the ERP signal that was measured from participants as they were presented with stimuli, and estimate how the ERP signal changes with increasing activation. Therefore, we can consider TRFs to be a viable method for analysing ERP data by a continuous variable, such as EDL activation values. As the TRFs do not provide information about statistical significance, we used a strict procedure to determine whether the fitted TRFs explain more variance of the ERP signal than baseline models that aggregate the data into categories such as high vs low tone and standard vs deviant stimuli, or stimulus frequency (i.e., the probability model).

\section{Discussion}

When listeners encounter speech sounds from an unfamiliar language, they often have difficulty discriminating them. However, discrimination can improve in the absence of explicit instruction, through implicit learning ${ }^{6}$. Recent evidence suggests that implicit learning occurs through a process of prediction and prediction error. The present study sought to determine whether evidence for such error-driven learning emerges in participants' trial-by-trial ERP signals as they listen to a sequence of lexical tones sampled from a bimodal pitch distribution. Below, we first discuss the results with the respect to our three research questions. We then discuss some implications for learning more generally, as well as our thoughts on the benefits of our method for ERP research.

\subsection{Trial-by-trial learning}

The first question we aimed to investigate in the present study was whether we could find evidence of trial-by-trial learning in the ERP signal. Error-driven learning simulations were used to obtain trial-by-trial estimates of participants' level of expectation of each incoming stimulus. These expectation estimates (activation values) were then used as input to the TRF modelling procedure, which generated estimates of the trialby-trial ERP waveforms in an early and a late time window. Rather than comparing discrete stimulus conditions, such as for example, standard vs. deviant or expected vs. unexpected stimuli, our EDL models predict gradient differences in the ERP depending on how expected the incoming stimulus is on each trial.

The TRF models derived from the EDL activations were compared to baseline models, with an additional safety check against a set of random models. Several EDL models were found to be more informative than their baselines. This finding shows that the EDL simulations captured trial-by-trial changes in expectation that were predictive of brain activity. As predicted, ERP amplitude increased with decreasing activation. In addition, several EDL models were also more informative than the probability model. Particularly in the early time window, the EDL models outperformed the probability model in several channels. This finding suggests that, at least in the early time window, the variation in the ERP signal captured by our expectation estimates does not result from the statistical distribution of the stimuli, but from error-driven learning.

${ }^{6}$ We use the term 'implicit learning' in the broad sense here, to refer to learning that occurs implicitly, rather than the somewhat more specific research field/paradigm by this name. 


\subsection{Learning representations and cognitive tasks}

The second question we aimed to investigate was what participants learn in this experiment. We started with the assumption that participants could potentially be learning on multiple levels simultaneously, as well as learning from potentially any discriminable cues. We designed simulations to model two cognitive tasks, categorisation and predicting the upcoming stimulus, with a variety of cues for estimating one of three outcome types: lexical tone (high vs. low), standard vs deviant or the specific pitch value (i.e. the pitch continuum step). Regarding this question, in some respects we cannot draw strong conclusions from the present study. Several EDL models were informative about the ERP signal and there was a substantial amount of overlap in the estimates of the different EDL simulations (see Figure 8). Since we did not formally compare the EDL models to each other, we cannot draw conclusions about which of the informative models are most informative.

However, we do note some patterns that are common between the informative models. Most of the informative EDL models predicted a gradual increase in expectation for the standards (stimuli 1-4) over the course of each block. This increase in expectation of the standard stimuli was captured by both the categorisation models (e.g. Cat-val-SD and Cat-diff-HL) and the prediction models (Pred-bg-HL, Pred-diff-HL, Pred-val-HL). Therefore, we cannot determine on the basis of this experiment whether learning was based on categorisation of the current stimulus or predicting the upcoming stimulus or both. However, the results do point to trial-by-trial learning that led to an increasing expectation of the stimuli over the course of each block. Many of the informative EDL models also predicted unlearning, i.e. a decrease in expectation, of the deviant (stimulus 5) over the course of the block, particularly the prediction models (Pred-bg-HL, Pred-diff-HL, Pred-val-HL).

The present experiment used a version of the oddball paradigm, in which, within each block, one stimulus is frequent and the other is comparatively rare. The foundation of the design of this paradigm is the assumption that the frequent stimulus will be expected and the rare stimulus will be unexpected. A wealth of literature (e.g. Frost et al., 2016; Haenschel et al., 2005; Kopp \& Wolff, 2000; Polich, 2011; Rushby et al., 2005) has shown that examining differences between these frequent and rare stimulus types gives us insight into the neural effects of expectation. However, the present results demonstrate that the effect is not categorical, but evolves over the course of the experiment and especially over the course of each block, as expectations develop. A consequence is that there will be cases where the standard stimulus (usually seen as an expected stimulus) is unexpected. And at least some of the EDL models, such as Pred-bg-HL, also predict that there will be the opposite case, trials on which the deviant stimuli are expected.

Indeed, while researchers typically conceptualise the oddball stimuli as standards and deviants, the participants in the experiment do not have direct access to these categories. The perceptual system only receives sensory information, such as the acoustic properties of the stimulus. Expectations can only develop over time, as information becomes available. The EDL modelling approach presented here allows us to model the overall difference in expectations between the frequent standard stimuli and the infrequent deviant stimuli, even when the models did not have access to these categories (as in all the HL models). Moreover, our EDL models not only capture the difference between standards and deviants, but also capture the gradual development of these expectations over time.

An interesting result is that the models predicting the upcoming pitch value (the 
val models, a subcategory of the prediction models) performed quite well in the early time window. In the present experiment, the pitch differences between continuum steps were very small. In fact, an offline test showed they were too small for a difference to be detected most of the time, even two steps apart on the continuum (Nixon et al., 2018). So it was interesting that models predicting the upcoming pitch value performed well. It is possible that these models pick up on perceptual sensitivity that is missed in the behavioural discrimination tasks, possibly even below the level of awareness. It should be noted that these models did not have the initial comparison against a baseline model, so the criteria for these val models were less strict than the other EDL model types. Nevertheless, they were found to be more informative than the probability models and outperformed the random models.

\subsection{ERP components, timing and scalp distribution}

The third question we aimed to investigate was the timing, location and shape of the ERP waveforms that are predicted by the trial-by-trial learning simulations. Generally speaking, evidence that the EDL models were more informative about the ERP response than the baseline models was found for the early time window, rather than for the late time window. In addition, we can draw some inferences based on the timing, polarity and scalp topography of the effects captured by our models. Both EDL types the prediction simulations, such as Pred-bg-HL, which predict the upcoming stimulus and the categorisation simulation Cat-diff-HL, which uses pitch changes to categorise stimuli into high or low lexical tones - lead to TRF models that predict a more negative component starting around $50 \mathrm{~ms}$ and peaking around $150 \mathrm{~ms}$ after onset with decreasing activation estimates. That is, the more surprising the stimulus according to the EDL simulation, the greater the ERP amplitude. The timing, polarity and localisation of this early negative-going peak correspond to the mismatch negativity response (Näätänen et al., 1978).

In the later time window, the prediction model, Pred-bg-HL, captures a more positive signal for lower activation estimates, especially after $400 \mathrm{~ms}$, at posterior and occipital electrodes. This is consistent with the P3a component (Kopp \& Wolff, 2000), which has been found to increase in amplitude in response to the level of surprise evoked by a stimulus, including unattended stimuli. The timing and posterior localisation are consistent with the P3a (Polich, 1988, 2011; Snyder \& Hillyard, 1976). This model was not more informative than the probability model, however.

Our models did not show evidence of predicting any effects akin to an N400, which is consistent with previous findings that the N400 is usually found in higher-level processing. Neither did we find evidence for effects comparable to error negativity $\mathrm{N}^{\mathrm{e}} /$ ERN) in any of the generated TRFs. The $\mathrm{N}^{\mathrm{e}}$ is typically associated with response error, which may explain why it was not found in this passive listening task.

Overall, the pattern of ERPs over the scalp and over time suggests 1) an early MMN-like component, which increases the more surprising a stimulus is in terms of its categorisation as a standard or deviant stimulus and in terms of its predictability in the sequence and 2) a late positive P3a-like component, which increases the more surprising an upcoming stimulus is based on the previous sequence. 


\subsection{Implications for learning}

Many studies have observed that the statistical structure of sensory or linguistic input can affect learning outcomes (Baldeweg, 2007; Frost et al., 2016; Maye \& Gerken, 2000; Maye et al., 2002; Nixon \& Best, 2018; Nixon et al., 2016; Saffran et al., 1996; Trotter et al., 2019). The present study examined whether error-driven learning (EDL) could be the mechanism underlying such statistical effects. The results show that EDL simulations are able to predict variation in the ERP signal during learning. The ERP amplitude differed depending on how expected an incoming stimulus was, as predicted by the EDL activation measure.

In the prediction task, neural activity was captured by a simulation that learned to predict upcoming lexical tones from a sequence of tones. In the categorisation task, neural activity was captured by a simulation that categorised pitch differences into lexical tones. Interestingly, despite the different input representations, these two models made similar predictions for the frequent stimuli, the standards. In both cases, the simulations predicted a gradual increase in expectation over the block and surprise (low expectation) at the beginning of a new block. However, by comparing the TRFs of these models to the TRFs of the standard-deviant baseline model (Figure 11), we discovered two interesting findings. Firstly, the TRF of categorisation model (Cat-diff-HL) suggests an ERP waveform that is somewhat distinct from the other cluster of correlated models, with an earlier peak and inflexion. And secondly, the prediction models capture variance in the ERPs that overlaps with, but is distinct from the standarddeviant baseline model. This suggests the possibility that the categorisation model and the standard-deviant baseline model might be capturing two distinct processes, which are both captured by the prediction models. In any case, both types of models were able to capture evidence of trial-by-trial learning in the ERPs. Moreover, most of these models did not have standards and deviants as input to the simulations, and yet comparison of their TRFs to the standard-deviant baseline model suggests these EDL simulations were still able to capture variance in the ERP signal corresponding to differences in expectation between the standard and deviant stimuli.

The results contribute to a growing literature suggesting that language is discriminative. For example, error-driven learning models have previously been used to model speech sound acquisition in the native language (Nixon \& Tomaschek, 2020, 2021) or a non-native language (Nixon, 2018, 2020), as well as word learning (Ramscar \& Baayen, 2013; Ramscar et al., 2010), word segmentation (Baayen, Shaoul, Willits, \& Ramscar, 2016), morphology (Hoppe et al., 2020) and articulation (Tomaschek, Plag, Ernestus, \& Baayen, 2021).

Note that we did not formally compare these simulations to the other models and that weaker correlations with the ERP signal also do not necessarily indicate simulations are incorrect. Similarly, the fact that EDL simulations were often more informative than overall statistics does not prove that overall statistics are not used at all; the probability model we used as a control model reflects a probability distribution over the whole learning phase. It is possible that after the learning phase, when participants have actually been exposed to the probability distribution, the probability model is more (and even most) informative of the ERP signal. It is possible that participants might slowly become more sensitive or less sensitive to the pitch continuum over the experiment (cf. Hoppe et al., 2022; Nixon \& Best, 2018; Nixon et al., 2016), which might mean that different models capture the experiment better at different times. In the present study, investigating this possibility would have added too much complexity to the models. Therefore, we leave this question for future research. 
The present study sheds light on some divergent results in distributional and statistical learning studies. While many studies report effects of distribution type on learning (unimodal vs. bimodal; e.g. Maye et al., 2002), a number of recent studies have found that learning does not always follow the predictions of purely frequency-based statistical learning. For example, Wanrooij et al. (2014) found differences in infants' post-test behaviour after training with unimodal verus bimodal distributions, but no differences were found for adults. They argue that adults lose the ability to learn from statistical learning. Error-driven learning predicts this phenomenon, because the most learning is expected to occur when uncertainty is greatest. Since infants have much less prior learning than adults, perceptual input tends to be more surprising in general. As learning occurs, the world becomes more predictable and there is less uncertainty left to drive learning. Secondly, it might be that adult participants use different information to what is being tested, because they have learned to pay attention to different cues or outcomes. That is, the actual cues and outcomes participants learn from might differ from those intended by the researchers.

\subsection{TRF fitting}

Our EDL simulations generated trial-by-trial estimates of listeners' expectations about upcoming stimuli. Our results show that these estimates can be used to predict the brain activity of these listeners, represented by the amplitude of the ERP signal. This was made possible by combining our EDL simulations with the method of fitting TRFs. TRF fitting has been used previously to predict ERPs (Brodbeck et al., 2018; Broderick, Anderson, Di Liberto, Crosse, \& Lalor, 2018); however, the present study is, to the best of our knowledge, the first to use TRF modelling to generate an ERP signal that changes dynamically over the course of the experiment, individually for each participant and each trial. The TRF fitting also allows us to investigate the timing and scalp distribution of the effects, discussed above.

A second innovation of the present study in terms of the TRF modelling was that we used strict criteria to test whether the EDL model contributed to the fit of the TRF-convolved input signal, by estimating the information loss of baseline models, a probability model and models based on EDL simulations. Our modelling approach is useful for experimental paradigms in which no baseline or control condition is available; all ERP data is used to assess the learning process. Our results show that our EDLderived estimates of the size of the spike (activation) in the input yield more informative TRFs than a baseline model spike (representing static properties of the stimulus), and in many cases also a probability model (representing the probability of the individual pitch stimuli). In addition, the sanity check against random models show that this is not because a better model is always possible, if one tries long enough. The fact that less (model, time window, electrode) combinations passed Test 3 than passed Test $1 / 2$ does not necessarily mean that random models are better than baseline models, but may very well reflect the limited power of the non-parametrized test against chance. Still, we argue the combination of tests used in the present study is valuable protection against spurious conclusions given the high (but unknown) degrees of freedom for both EDL simulations and TRF fitting.

Our approach does not allow us to directly compare the EDL models against each other, because the EDL models are not considered independent. However, it is powerful enough to test twenty models at once, with a strong Bonferroni correction not eliminating all significance. Although the TRFs do not show which model fits best, they do 
show for the models that predict the ERP signal how these models fit to the signal. In addition, our approach is novel in the strict control of the information explained by the EDL estimates alone. If we had only used an EDL-derived input signal, without the separate constant dimension, any significant fit might be (largely) explained by information provided by the time-locking of the spikes to the stimuli, making it impossible to determine the quality of the EDL simulation. This aspect of the modelling, which controls for the effect of the time-locking of spikes to any stimulus, is an improvement over previous studies that have used TRF modelling (see also Ahmed, 2021). Future studies that use TRF modelling could consider including a constant dimension to disentangle the effect of any stimulus from the particular predictor of interest under study.

\subsection{Conclusion}

In summary, the EDL simulations produced activation values that produced more informative models of the ERP signal than comparable models without EDL estimates. The extra information that allows for a better prediction of the ERP signal must thus be based on the error-driven learning predictions and is over and above changes in the ERP signal resulting from incoming auditory signal in general or static properties of the stimuli. In many cases, the EDL models also outperformed models based on distribution frequency.

Error-driven learning (EDL) simulations combined with temporal-response function (TRF) fitting allowed us to investigate the trial-by-trial effects of learning on ERPs. We were able to do this without a-priori assumptions about the ERP components. The EDL predictions successfully captured how trial-by-trial learning affects brain activity. In addition, the results suggest that learning may occur on multiple levels simultaneously. An innovation in our approach to TRF fitting enabled us to tease apart expectation and learning effects from mere acoustic processing and other effects on the ERPs. We propose that the combination of these two methods provides a promising approach to the investigation of neural processes underlying learning.

\section{Acknowledgements}

We would like to thank two anonymous reviewers for helpful and constructive feedback, which has helped to improve the manuscript. We would also like to thank the organisers of the 'Modelling meets Infant Studies in Language Acquisition: A Dialogue on Current Challenges and Future Directions' Workshop (2013), Lorentz-Centre, Leiden, The Netherlands. This paper is a spin-off of the collaborative project that started at this workshop. Thanks also to our other collaborators Natalie Boll-Avetisyan, Sandrien van Ommen, Çağri Çöltekin, Brigitta Keij, and Liquan Liu for their input and the possibility to use the data we collected together. Further, we would also like to thank Rasyan Ahmed for allowing us to reuse parts of his Python code. This research was supported an AMT Individual Researcher Grant (JSN), an ERC Advanced Grant (Grant number 742545) and the Netherlands Organisation for Scientific Research NWO (Veni grant no. $275-70-044, \mathrm{JvR}$ ). 


\section{References}

Ahmed, R. (2021). Modeling EEG responses to narrative speech (Unpublished master's thesis). University of Amsterdam, Amsterdam.

Baayen, R. H., Milin, P., Đurđević, D. F., Hendrix, P., \& Marelli, M. (2011). An amorphous model for morphological processing in visual comprehension based on naive discriminative learning. Psychological Review, 118(3), 438. doi:

Baayen, R. H., Shaoul, C., Willits, J., \& Ramscar, M. (2016). Comprehension without segmentation: A proof of concept with naive discriminative learning. Language, Cognition and Neuroscience, 31(1), 106-128.

Baldeweg, T. (2007). ERP repetition effects and mismatch negativity generation: a predictive coding perspective. Journal of Psychophysiology, 21(3-4), 204-213. doi:

Besson, M., \& Macar, F. (1987). An eventrelated potential analysis of incongruity in music and other nonlinguistic contexts. Psychophysiology, 24(1), 14-25. doi:

Boersma, P., \& Weenink, D. (2014). Praat (version 5.5).

Boll-Avetisyan, N., Nixon, J. S., Lentz, T. O., Liu, L., van Ommen, S., Cöltekin, C., \& van Rij, J. (2018). Neural response development during distributional learning. Proceedings of Interspeech 2018, 1432-1436. doi:

Brodbeck, C., Presacco, A., \& Simon, J. Z. (2018). Neural source dynamics of brain responses to continuous stimuli: Speech processing from acoustics to comprehension. NeuroImage, 172, 162-174. doi:

Broderick, M. K., Anderson, A. J., Di Liberto, G. M., Crosse, M. J., \& Lalor, E. C. (2018). Electrophysiological correlates of semantic dissimilarity reflect the comprehension of natural, narrative speech. Current Biology, 28(5), 803-809.E3. doi:

Bröker, F., \& Ramscar, M. (2020). Representing absence of evidence: why algorithms and representations matter in models of language and cognition. Language, Cognition and Neuroscience, 1-24.

Costa-Faidella, J., Grimm, S., Slabu, L., Díaz-Santaella, F., \& Escera, C. (2011). Multiple time scales of adaptation in the auditory system as revealed by human evoked potentials. Psychophysiology, 48(6), 774-783. doi:

de Cat, C., Klepousniotou, E., \& Baayen, H. (2015). Representational deficit or processing effect? An electrophysiological study of noun-noun compound processing by very advanced L2 speakers of English. Frontiers in Psychology, 6, 77.

Falkenstein, M., Hohnsbein, J., Hoormann, J., \& Blanke, L. (1991). Effects of crossmodal divided attention on late ERP components. II. Error processing in choice reaction tasks. Electroencephalography and clinical neurophysiology, 78(6), 447455. doi:

Frost, J. D., Winkler, I., Provost, A., \& Todd, J. (2016). Surprising sequential effects on MMN. Biological Psychology, 116, 47-56. doi:

Gramfort, A., Luessi, M., Larson, E., Engemann, D., Strohmeier, D., Brodbeck, C., ... Hämäläinen, M. (2013). MEG and EEG data analysis with MNE-Python. Frontiers in Neuroscience, \%. doi:

Haenschel, C., Vernon, D. J., Dwivedi, P., Gruzelier, J. H., \& Baldeweg, T. (2005). Event-related brain potential correlates of human auditory sensory memory-trace formation. Journal of Neuroscience, 25 (45), 10494-10501. doi:

Hasson, U. (2017). The neurobiology of uncertainty: Implications for statistical learning. Philosophical Transactions of the Royal Society B: Biological Sciences, 372(1711), 20160048. doi: 
Holroyd, C., Nieuwenhuis, S., Mars, R. B., \& Coles, M. G. H. (2004). Anterior cingulate cortex, selection for action, and error processing. In M. I. Posner (Ed.), Cognitive neuroscience of attention (pp. 219-231). New York, NY: Guilford Publishing.

Hoppe, D. B., Hendriks, P., Ramscar, M., \& van Rij, J. (2022). An exploration of error-driven learning in simple two-layer networks from a discriminative learning perspective. doi:

Hoppe, D. B., van Rij, J., Hendriks, P., \& Ramscar, M. (2020). Order matters! influences of linear order on linguistic category learning. Cognitive Science, $44(11)$, e12910.

Kamin, L. J. (1969). Predictability, surprise, attention and conditioning. In B. A. Campbell \& R. M. Church (Eds.), Punishment and aversive behavior (pp. 279-296). New York, NY: Appleton-Century-Crofts.

Koelsch, S., Busch, T., Jentschke, S., \& Rohrmeier, M. (2016). Under the hood of statistical learning: A statistical MMN reflects the magnitude of transitional probabilities in auditory sequences. Scientific Reports, 6, 19741. doi:

Kopp, B., \& Wolff, M. (2000). Brain mechanisms of selective learning: Event-related potentials provide evidence for error-driven learning in humans. Biological Psychology, 51 (2-3), 223-246. doi:

Kutas, M., \& Federmeier, K. D. (2011). Thirty years and counting: Finding meaning in the N400 component of the event-related brain potential (ERP). Annual Review of Psychology, 62, 621-647. doi:

Kutas, M., \& Hillyard, S. A. (1980). Reading senseless sentences: Brain potentials reflect semantic incongruity. Science, 207(4427), 203-205. doi:

Lalor, E. C., \& Foxe, J. J. (2010). Neural responses to uninterrupted natural speech can be extracted with precise temporal resolution. European Journal of Neuroscience, 31, 189-193. doi:

Maye, J., \& Gerken, L. (2000). Learning phonemes without minimal pairs. In S. C. Howell, S. A. Fish, \& T. Keith-Lucas (Eds.), BUCLD 24 (24th Annual Boston University Conference on Language Development) Proceedings (Vol. 2, pp. 522-533). Somerville, MA: Cascadilla Press.

Maye, J., Werker, J. F., \& Gerken, L. (2002). Infant sensitivity to distributional information can affect phonetic discrimination. Cognition, 82(3), B101-B111. doi:

Miltner, W. H., Braun, C. H., \& Coles, M. G. (1997). Event-related brain potentials following incorrect feedback in a time-estimation task: Evidence for a "generic" neural system for error detection. Journal of Cognitive Neuroscience, 9(6), 788798. doi:

Näätänen, R., Gaillard, A. W. K., \& Mäntysalo, S. (1978). Early selective-attention effect on evoked potential reinterpreted. Acta Psychologica, 42(4), 313-329. doi:

Näätänen, R., \& Kreegipuu, K. (2011). The mismatch negativity (MMN). In S. J. Luck \& E. S. Kappenman (Eds.), The Oxford handbook of event-related potential components. New York, NY: Oxford University Press.

Nieuwland, M. S., \& van Berkum, J. J. A. (2006). When peanuts fall in love: N400 evidence for the power of discourse. Journal of Cognitive Neuroscience, 18(7), 1098-1111. doi:

Nixon, J. S. (2014). Sound of mind: electrophysiological and behavioural evidence for the role of context, variation and informativity in human speech processing. Doctoral dissertation, University of Leiden, Netherlands.

Nixon, J. S. (2018, September). Effective acoustic cue learning is not just statistical, it is discriminative. In Interspeech $2018-19^{\text {th }}$ Annual Conference of the 
International Speech Communication Association (pp. 1447 - 1451). Hyderabad, India.

Nixon, J. S. (2020). Of mice and men: Speech sound acquisition as discriminative learning from prediction error, not just statistical tracking. Cognition, 197, 104081. doi:

Nixon, J. S., \& Best, C. T. (2018, June). Acoustic cue variability affects eye movement behaviour during non-native speech perception. In Proceedings of Speech Prosody (pp. 493-497). Poznan, Poland.

Nixon, J. S., Boll-Avetisyan, N., Lentz, T. O., van Ommen, S., Keij, B., Cöltekin, C., ... van Rij, J. (2018). Short-term exposure enhances perception of both betweenand within-category acoustic information. In Proceedings of the 9th International Conference on Speech Prosody (pp. 114-118). doi:

Nixon, J. S., Poelstra, S., \& van Rij, J. (2022). Does error-driven learning occur in the absence of cues? Examination of the effects of updating connection weights to absent cues. In Proceedings of the 44th Annual Meeting of the Cognitive Science Society.

Nixon, J. S., \& Tomaschek, F. (2020). Learning from the acoustic signal: Errordriven learning of low-level acoustics discriminates vowel and consonant pairs. In S. Denison, M. Mack, Y. Xu, \& B. C. Armstrong (Eds.), Proceedings of CogSci 2020, the 42nd annual meeting of the cognitive science society (pp. 585-591). Cognitive Science Society.

Nixon, J. S., \& Tomaschek, F. (2021). Prediction and error in early infant speech perception: A speech acquisition model. Cognition, 212, 104697.

Nixon, J. S., van Rij, J., Li, X. Q., \& Chen, Y. (2015). Cross-category phonological effects on ERP amplitude demonstrate context-specific processing during reading aloud. In A. Botonis (Ed.), ExLing 2015: Proceedings of the International Conference of Experimental Linguistics (pp. 50-53).

Nixon, J. S., van Rij, J., Mok, P., Baayen, R. H., \& Chen, Y. (2016). The temporal dynamics of perceptual uncertainty: eye movement evidence from Cantonese segment and tone perception. Journal of Memory and Language, 90, 103-125.

Olejarczuk, P., Kapatsinski, V., \& Baayen, R. H. (2018). Distributional learning is error-driven: The role of surprise in the acquisition of phonetic categories. Linguistics Vanguard, 4(s2). doi:

Oostenveld, R., Fries, P., Maris, E., \& Schoffelen, J.-M. (2011). FieldTrip: Open source software for advanced analysis of MEG, EEG, and invasive electrophysiological data. Computational Intelligence and Neuroscience, 2011, 1. doi:

Perruchet, P., \& Pacton, S. (2006). Implicit learning and statistical learning: One phenomenon, two approaches. Trends in Cognitive Sciences, 10(5), 233-238. doi:

Polich, J. (1988). Bifurcated P300 peaks: P3a and P3b revisited? Journal of Clinical Neurophysiology, 5(3), 287-294.

Polich, J. (2011). Neuropsychology of P300. In S. J. Luck \& E. S. Kappenman (Eds.), The Oxford handbook of event-related potential components. New York, NY: Oxford University Press.

Ramscar, M., \& Baayen, H. R. (2013). Production, comprehension, and synthesis: A communicative perspective on language. Frontiers in Psychology, 4(233). doi:

Ramscar, M., Dye, M., \& McCauley, S. M. (2013). Error and expectation in language learning: The curious absence of mouses in adult speech. Language, 89(4), 760793.

Ramscar, M., \& Yarlett, D. (2007). Linguistic self-correction in the absence of feedback: 
A new approach to the logical problem of language acquisition. Cognitive Science, 31(6), 927-960.

Ramscar, M., Yarlett, D., Dye, M., Denny, K., \& Thorpe, K. (2010). The effects of feature-label-order and their implications for symbolic learning. Cognitive Science, 34 (6), 909-957. doi:

Reber, A. S. (1989). Implicit learning and tacit knowledge. Journal of Experimental Psychology: General, 118(3), 219. doi:

Rebuschat, P., \& Monaghan, P. (2019). Editors' introduction: Aligning implicit learning and statistical learning: Two approaches, one phenomenon. Topics in cognitive science, 11(3), 459-467.

Rescorla, R. A., \& Wagner, A. R. (1972). A theory of Pavlovian conditioning: variations in the effectiveness of reinforcement and nonreinforcement. In A. H. Black \& W. F. Prokasy (Eds.), Classical conditioning II: Current research and theory (pp. 64-99). New York, NY: Appleton-Century-Crofts.

Rushby, J. A., Barry, R. J., \& Doherty, R. J. (2005). Separation of the components of the late positive complex in an ERP dishabituation paradigm. Clinical Neurophysiology, 116(10), 2363-2380. doi:

Saffran, J. R., Aslin, R. N., \& Newport, E. L. (1996). Statistical learning by 8-monthold infants. Science, 274(5294), 1926-1928. doi:

Saffran, J. R., \& Kirkham, N. Z. (2018). Infant statistical learning. Annual Review of Psychology, 69. doi:

Shannon, C. E. (1948). A mathematical theory of communication. Bell System Technical Journal, 27.

Sitnikova, T., Kuperberg, G., \& Holcomb, P. J. (2003). Semantic integration in videos of real-world events: An electrophysiological investigation. Psychophysiology, 40(1), 160-164. doi:

Snyder, E., \& Hillyard, S. A. (1976). Long-latency evoked potentials to irrelevant, deviant stimuli. Behavioral Biology, 16(3), 319-331. doi:

Tomaschek, F., Plag, I., Ernestus, M., \& Baayen, R. H. (2021). Phonetic effects of morphology and context: Modeling the duration of word-final s in english with naïve discriminative learning. Journal of Linguistics, 57(1), 123-161.

Tremblay, A., \& Baayen, R. H. (2010). Holistic processing of regular four-word sequences: A behavioral and ERP study of the effects of structure, frequency, and probability on immediate free recall. In D. Wood (Ed.), Perspectives on formulaic language: Acquisition and communication (pp. 151-173). London: Continuum.

Trotter, A. S., Monaghan, P., Beckers, G. J., \& Christiansen, M. H. (2019). Exploring variation between artificial grammar learning experiments: Outlining a metaanalysis approach. Topics in Cognitive Science. doi:

Wanrooij, K., Boersma, P., \& Benders, T. (2015). Observed effects of "distributional learning" may not relate to the number of peaks. a test of "dispersion" as a confounding factor. Frontiers in psychology, 6, 1341.

Wanrooij, K., Boersma, P., \& van Zuijen, T. L. (2014). Distributional vowel training is less effective for adults than for infants. a study using the mismatch response. PLoS ONE, 9(10). doi:

Widrow, B., \& Hoff, M. E. (1960/1988). Adaptive switching circuits. In J. A. Anderson \& E. Rosenfeld (Eds.), Neurocomputing: Foundations of Research (pp. 96-104). MIT Press. (Reprint of 1960 technical report)

Winkler, I., Kujala, T., Tiitinen, H., Sivonen, P., Alku, P., Lehtokoski, A., ... Näätänen, R. (1999). Brain responses reveal the learning of foreign language phonemes. Psychophysiology, 36(5), 638-642. doi: 
This section contains visualizations of the data.

\section{a. Grand averages FC6}

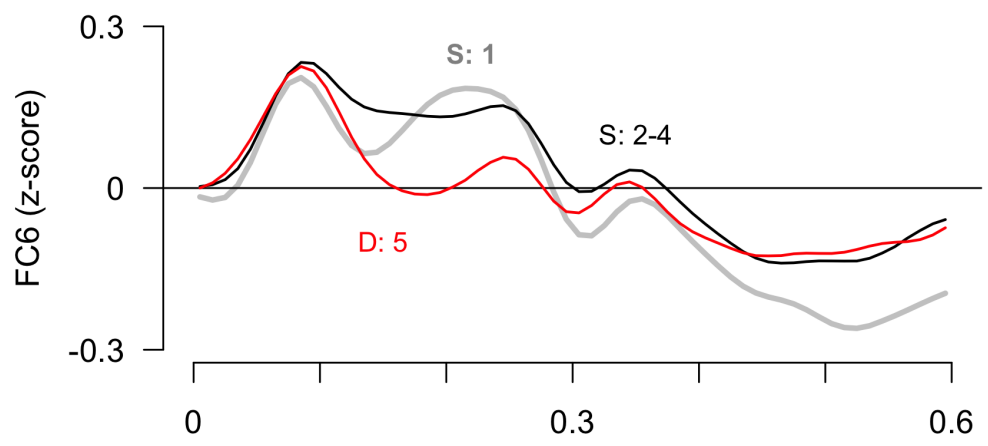

Time (s)

\section{b. Grand averages $\mathrm{Pz}$}

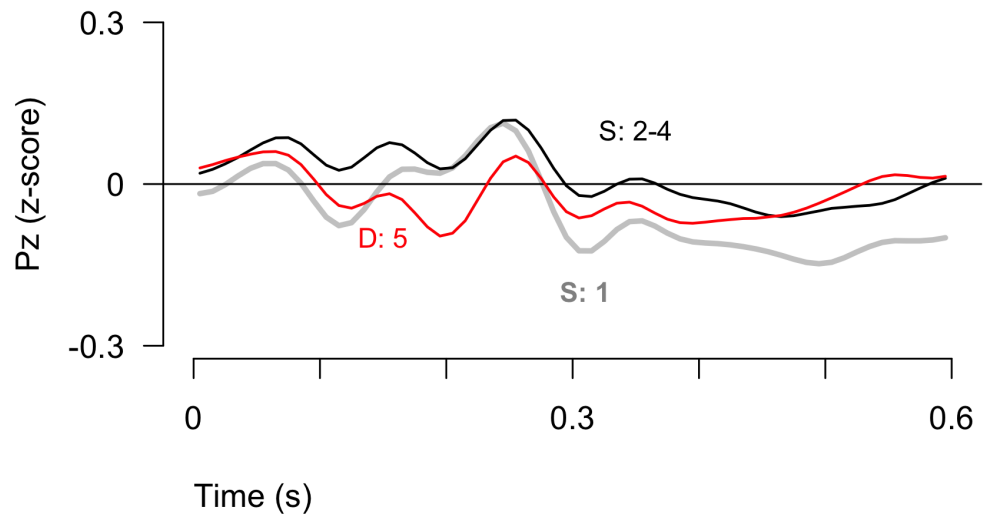

Figure A1. Grand averages of the z-scores of the ERP data in FC6 $(a)$ and Pz (b). 'S: 1' = first standard; 'S: $2-4$ ' = standards, sounds $2,3,4 ;$ 'D: 5' = deviant, fifth sound. 
Channel FC6:

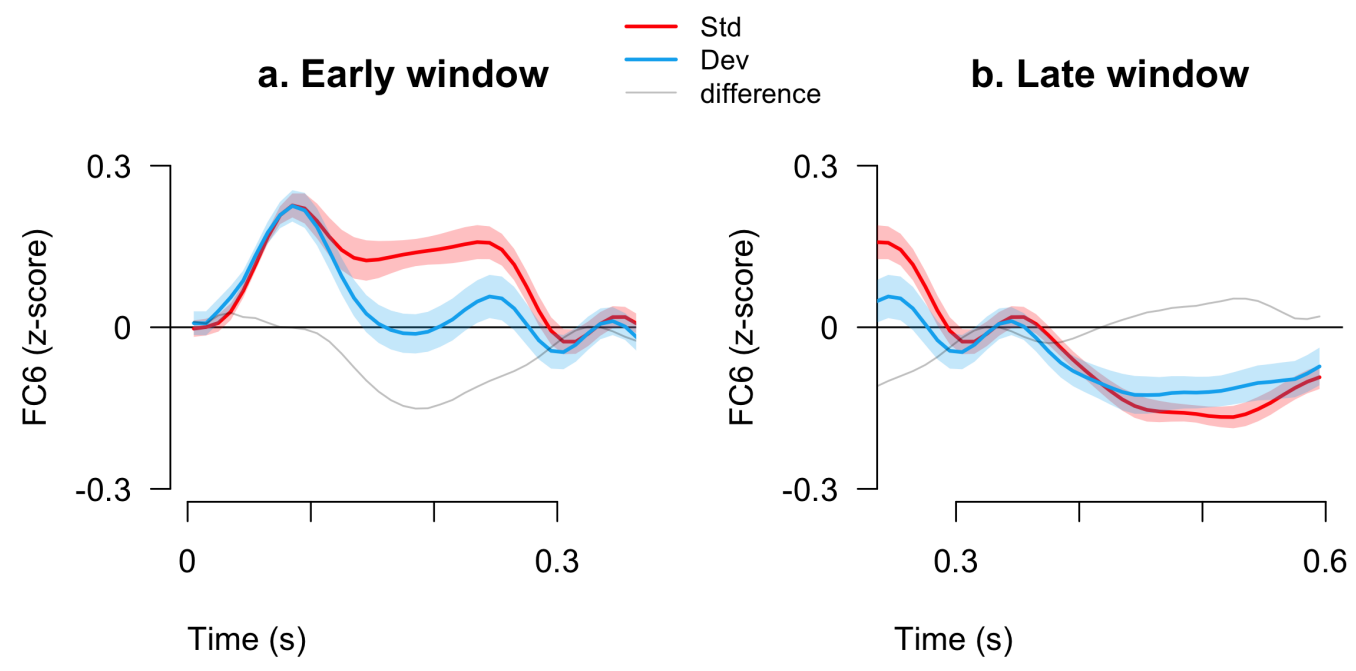

Channel Pz:

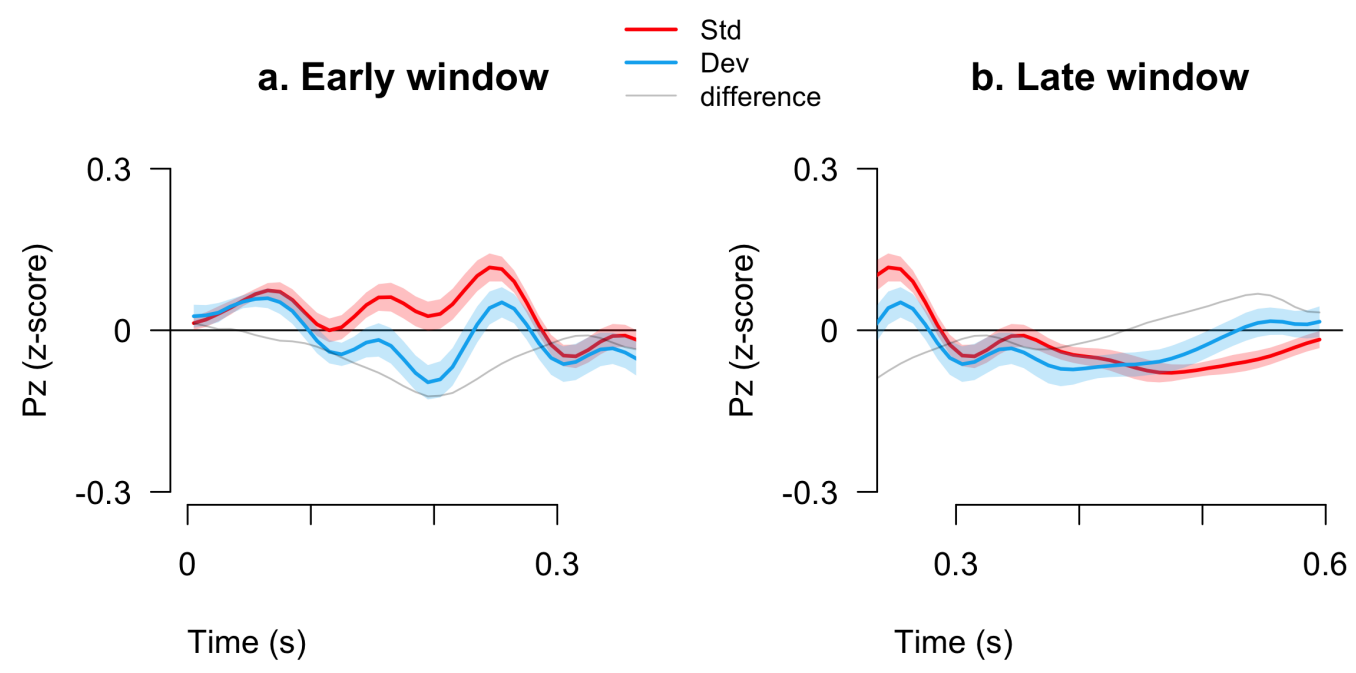

Figure A2. Grand averages of the z-scores of the ERP data in FC6 (top row) and Pz (bottom row), for standards and deviants. Panels a and b show two (overlapping) analysis time windows. Panel a: Early time window from $0-0.35 \mathrm{~s}$ after sound onset. Panel $b$ : Late time window from $0.25-0.6 \mathrm{~s}$ after sound onset. 
1607 Appendix B. Full comparison of models against random models 
Table B1. Proportion of random models beaten for each EDL model, in each time window and for each electrode selected in the previous step. Proportion of participants for whom the same comparison was also significant is given between brackets. Bonferroni corrected alpha levels for 64 ( 32 channels $\times 2$ time windows) comparisons, values are only shown if $\mathrm{p}<0.00078125$. As this was never the case for model Cat-pos-SD, its (empty) column has been deleted. * Model Cat-val-HL never beat its baseline and thus is not considered further, but is left in for transparency.

\begin{tabular}{|c|c|c|c|c|c|c|c|c|c|c|c|c|c|c|}
\hline \multicolumn{15}{|c|}{ Time window $0-350 \mathrm{~ms}$} \\
\hline \multirow[b]{2}{*}{ Channel } & \multicolumn{14}{|c|}{ Model } \\
\hline & Cat-diff-HL & Cat-diff-SD & Cat-pos-HL & (Cat-val-HL*) & Cat-val-SD & Pred-bg-HL & Pred-bg-SD & Pred-bg-VAL & Pred-diff-HL & Pred-diff-SD & Pred-diff-VAL & Pred-val-HL & Pred-val-SD & Pred-val-VAL \\
\hline F7 & $0.55(0.6)$ & $0.67(0.8)$ & $0.77(0.8)$ & & $0.88(1.0)$ & $0.88(1.0)$ & $0.69(0.8)$ & $0.62(0.6)$ & $0.79(0.8)$ & $0.56(0.5)$ & $0.61(0.6)$ & $0.78(0.8)$ & $0.68(0.8)$ & $0.59(0.6)$ \\
\hline FC5 & $0.61(0.7)$ & $0.62(0.5)$ & $0.81(0.9)$ & & $\mathbf{0 . 8 9}(0.9)$ & $0.87(0.9)$ & $0.61(0.6)$ & $0.66(0.8)$ & $0.78(0.8)$ & $0.61(0.6)$ & $0.63(0.6)$ & $0.83(0.9)$ & $0.63(0.6)$ & $0.65(0.6)$ \\
\hline $\mathrm{T} 7$ & $0.51(0.5)$ & $0.63(0.7)$ & $0.63(0.7)$ & & $\mathbf{0 . 6 9}(0.8)$ & $0.68(0.7)$ & $0.67(0.6)$ & $0.60(0.6)$ & $0.64(0.7)$ & $0.59(0.7)$ & $0.63(0.7)$ & $0.64(0.7)$ & $0.59(0.6)$ & $0.65(0.7)$ \\
\hline $\mathrm{C} 3$ & $0.61(0.6)$ & $0.79(0.8)$ & $0.74(0.8)$ & & $0.83(0.9)$ & $\mathbf{0 . 8 4}(0.9)$ & $0.68(0.7)$ & $0.67(0.7)$ & $0.77(0.8)$ & $0.61(0.6)$ & $0.67(0.7)$ & $0.82(0.8)$ & $0.65(0.6)$ & $0.69(0.8)$ \\
\hline $\mathrm{CP} 1$ & $0.55(0.5)$ & $0.68(0.7)$ & $0.68(0.7)$ & & $0.75(0.8)$ & $\mathbf{0 . 7 5}(0.8)$ & $0.58(0.6)$ & $0.67(0.8)$ & $0.74(0.8)$ & $0.59(0.6)$ & $0.56(0.6)$ & $0.73(0.8)$ & $0.63(0.6)$ & $0.59(0.6)$ \\
\hline CP5 & $0.52(0.4)$ & $\mathbf{0 . 7 2}(0.8)$ & $0.63(0.6)$ & & $0.70(0.8)$ & $0.70(0.8)$ & $0.69(0.8)$ & $0.61(0.6)$ & $0.67(0.7)$ & $0.55(0.6)$ & $0.64(0.8)$ & $0.64(0.6)$ & $0.56(0.6)$ & $0.66(0.7)$ \\
\hline P3 & $0.64(0.8)$ & $\mathbf{0 . 6 9}(0.8)$ & $0.64(0.7)$ & & $0.67(0.8)$ & $0.68(0.8)$ & $0.61(0.6)$ & $0.60(0.6)$ & $0.67(0.6)$ & $0.53(0.6)$ & $0.53(0.5)$ & $0.66(0.7)$ & $0.57(0.5)$ & $0.53(0.3)$ \\
\hline $\mathrm{Pz}$ & $0.65(0.7)$ & $0.62(0.6)$ & $0.63(0.6)$ & & $0.65(0.8)$ & $0.64(0.7)$ & $0.58(0.6)$ & $0.57(0.5)$ & $\mathbf{0 . 6 6}(0.8)$ & & & $0.64(0.6)$ & $0.55(0.5)$ & \\
\hline $\mathrm{PO} 3$ & $0.64(0.6)$ & $0.66(0.7)$ & $0.60(0.5)$ & & $0.65(0.8)$ & $0.64(0.8)$ & $0.56(0.6)$ & $0.60(0.6)$ & $0.59(0.6)$ & & & $0.60(0.6)$ & $0.57(0.5)$ & $0.53(0.6)$ \\
\hline $\mathrm{Oz}$ & $\mathbf{0 . 6 6}(0.6)$ & $0.60(0.6)$ & $0.51(0.4)$ & & $0.57(0.6)$ & $0.57(0.6)$ & & $0.61(0.6)$ & & & & $0.51(0.4)$ & & $0.51(0.4)$ \\
\hline $\mathrm{O} 2$ & $\mathbf{0 . 6 5}(0.7)$ & $0.62(0.7)$ & $0.52(0.4)$ & & $0.63(0.7)$ & $0.60(0.7)$ & $0.56(0.6)$ & $0.54(0.5)$ & & & & $0.55(0.6)$ & & \\
\hline P4 & $0.56(0.5)$ & $0.67(0.7)$ & $0.69(0.7)$ & & $0.67(0.8)$ & $0.72(0.8)$ & $0.60(0.7)$ & $0.61(0.6)$ & $0.74(0.8)$ & $0.55(0.6)$ & $0.53(0.4)$ & $\mathbf{0 . 7 6}(0.8)$ & $0.58(0.6)$ & $0.55(0.5)$ \\
\hline $\mathrm{CP} 2$ & $0.59(0.6)$ & $0.74(0.8)$ & $0.72(0.7)$ & & $0.70(0.8)$ & $0.74(0.8)$ & $0.61(0.6)$ & $0.62(0.7)$ & $\mathbf{0 . 7 8}(0.8)$ & $0.56(0.6)$ & $0.56(0.4)$ & $0.77(0.8)$ & $0.59(0.6)$ & $0.53(0.4)$ \\
\hline $\mathrm{T} 8$ & $0.57(0.4)$ & $0.68(0.6)$ & $0.84(0.9)$ & $0.52(0.5)$ & $0.81(0.9)$ & $0.86(0.9)$ & $0.71(0.6)$ & $0.85(0.9)$ & $0.81(0.9)$ & $\mathbf{0 . 8 7}(0.9)$ & $0.80(0.9)$ & $0.84(0.9)$ & $0.85(0.9)$ & $0.74(0.7)$ \\
\hline FC6 & $0.62(0.6)$ & $0.82(0.9)$ & $0.89(0.9)$ & & $0.94(1.0)$ & $0.92(1.0)$ & $0.70(0.7)$ & $0.84(0.9)$ & $0.89(0.9)$ & $0.76(0.9)$ & $0.74(0.7)$ & $0.89(0.9)$ & $0.76(0.9)$ & $0.77(0.8)$ \\
\hline F8 & $0.69(0.7)$ & $0.68(0.8)$ & $0.76(0.7)$ & & $\mathbf{0 . 8 6}(0.9)$ & $0.84(0.9)$ & $0.67(0.6)$ & $0.84(0.9)$ & $0.75(0.7)$ & $0.76(0.8)$ & $0.73(0.7)$ & $0.81(0.8)$ & $0.64(0.5)$ & $0.76(0.8)$ \\
\hline \multicolumn{15}{|c|}{ Time window $250-600 \mathrm{~ms}$} \\
\hline & \multicolumn{14}{|c|}{ Model } \\
\hline Channel & Cat-diff-HL & Cat-diff-SD & Cat-pos-HL & Cat-val-HL & Cat-val-SD & Pred-bg-HL & Pred-bg-SD & Pred-bg-VAL & Pred-diff-HL & Pred-diff-SD & Pred-diff-VAL & Pred-val-HL & Pred-val-SD & Pred-val-VAL \\
\hline P3 & $\mathbf{0 . 6 6}(0.6)$ & $0.52(0.4)$ & $0.62(0.7)$ & & $0.61(0.5)$ & $0.64(0.6)$ & & $0.62(0.7)$ & $0.60(0.6)$ & $0.63(0.6)$ & $0.61(0.8)$ & $0.62(0.6)$ & $0.59(0.6)$ & $0.60(0.6)$ \\
\hline $\mathrm{Pz}$ & $\mathbf{0 . 7 2}(0.8)$ & & $0.58(0.6)$ & & $0.60(0.6)$ & $0.63(0.7)$ & & $0.63(0.7)$ & $0.52(0.4)$ & $0.62(0.6)$ & $0.59(0.6)$ & $0.57(0.5)$ & $0.56(0.6)$ & $0.57(0.6)$ \\
\hline PO3 & $\mathbf{0 . 6 9}(0.8)$ & $0.52(0.4)$ & $0.62(0.7)$ & & $0.69(0.7)$ & $0.68(0.7)$ & & $0.67(0.8)$ & $0.55(0.6)$ & $0.62(0.6)$ & $0.62(0.6)$ & $0.60(0.6)$ & $0.61(0.6)$ & $0.64(0.7)$ \\
\hline $\mathrm{O} 1$ & $0.69(0.7)$ & $0.56(0.5)$ & $0.65(0.8)$ & & $\mathbf{0 . 7 3}(0.8)$ & $0.73(0.8)$ & & $0.65(0.7)$ & $0.59(0.6)$ & $0.62(0.6)$ & $0.58(0.7)$ & $0.62(0.6)$ & $0.64(0.6)$ & $0.58(0.6)$ \\
\hline $\mathrm{Oz}$ & $\mathbf{0 . 6 8}(0.7)$ & & $0.56(0.6)$ & & $0.63(0.8)$ & $0.62(0.6)$ & & $0.56(0.6)$ & $0.54(0.6)$ & & $0.59(0.6)$ & $0.55(0.6)$ & $0.58(0.5)$ & $0.59(0.8)$ \\
\hline $\mathrm{O} 2$ & $\mathbf{0 . 6 7}(0.8)$ & $0.57(0.5)$ & $0.59(0.6)$ & & $0.66(0.7)$ & $0.62(0.6)$ & & $0.51(0.4)$ & $0.55(0.5)$ & & $0.57(0.6)$ & $0.54(0.5)$ & & $0.52(0.5)$ \\
\hline PO4 & $0.66(0.8)$ & $0.60(0.6)$ & $0.65(0.8)$ & & $\mathbf{0 . 6 6}(0.8)$ & $0.65(0.7)$ & $0.56(0.5)$ & $0.62(0.7)$ & $0.63(0.7)$ & $0.59(0.6)$ & $0.59(0.6)$ & $0.62(0.6)$ & $0.61(0.6)$ & $0.63(0.7)$ \\
\hline
\end{tabular}




\section{Appendix C. Comparison against the Probability model}

In Test 2, the EDL models were compared against the Probability model. Note that the Probability model is the baseline model for the EDL models with the 'val' outcome (i.e., predicting the pitch values), and therefore the results of Test 1 and Test2 are identical for this group of EDL models.

a. Cat. Models:

\section{Early time window}
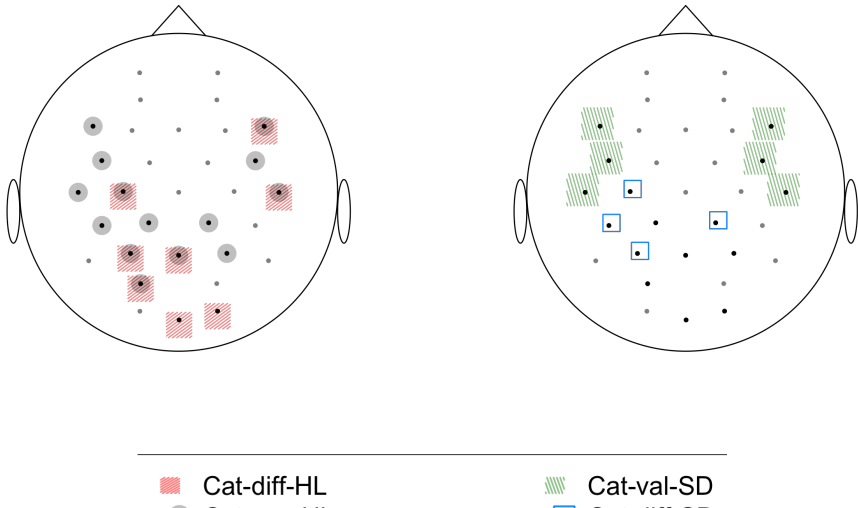

Cat-pos-HL
(IIII) Cat-val-SD

$\square$ Cat-diff-SD
Late time window

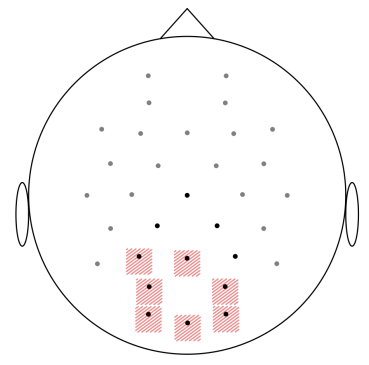

Cat-diff-HL

Cat-pos-HL

ilili Cat-val-SD

b. Pred. Models:

Early time window
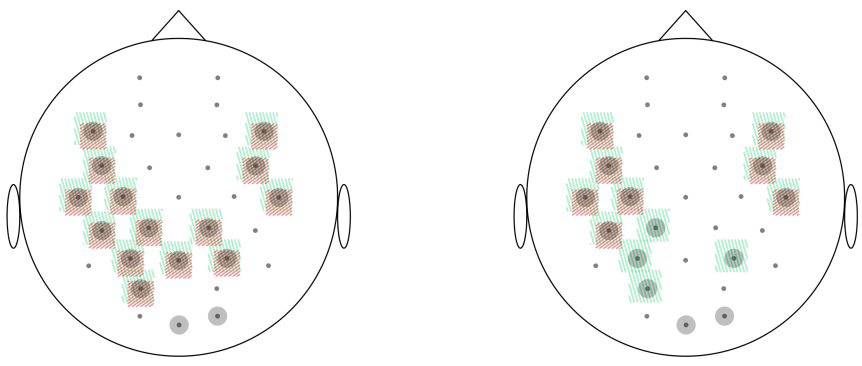

Pred-val-HL
Pred-diff-HL
Pred-bg-HL
Pred-val-VAL
Pred-diff-VAL
Pred-bg-VAL
Late time window

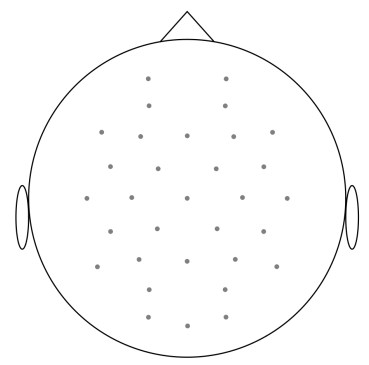

Figure C1. Channel/time windows combinations for which EDL models beat the Probability model (i.e., Test 2), and significantly more than $50 \%$ of the random models (based on Bonferroni corrected alpha level for 64 (32 channels $\times 2$ time windows) comparisons, values only shown if $p<0.00078125)$. Top row a) shows the categorization models, bottom row b) shows the prediction models. 


\section{Appendix D. Predictions of EDL models}

Below are the activation patterns visualised for different EDL models:

1615

1616

1617

1618

1619

1620

1621

- Figures D1 shows a group of EDL models with similar activation patterns, which were found to be informative for the ERP data. (Note that one of these is included in Figure D2, topleft because of space limitations.)

- Figure D2 shows a group of EDL models with activation patterns that were not correlated, which were found to be informative for the ERP data.

- Figure D3 shows another group of EDL models with similar activation patterns, which were also found to be informative for the ERP data. 
Pred-bg-HL [1]

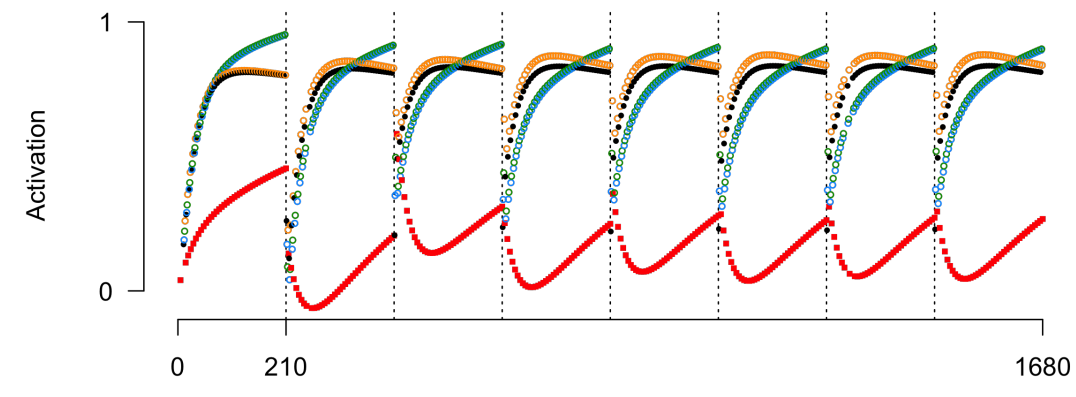

Input $\rightarrow$

Pred-val-HL [1]

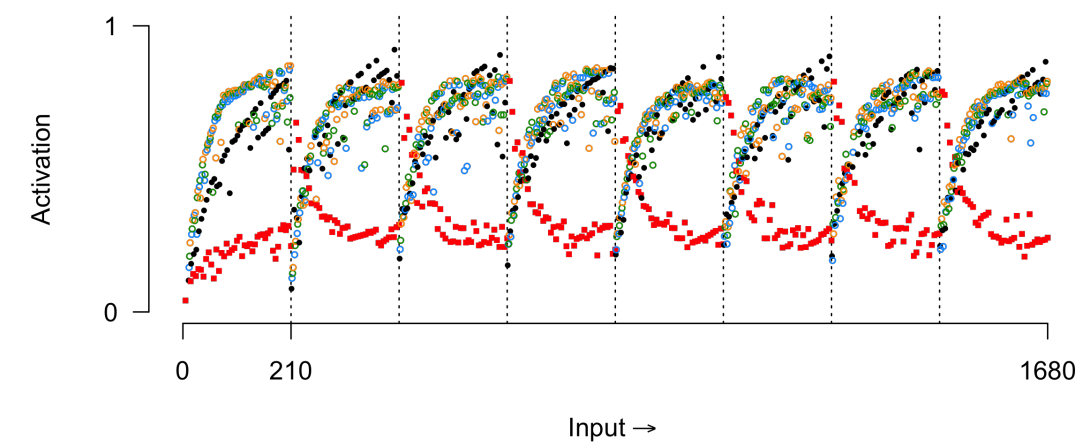

Pred-diff-HL [1]

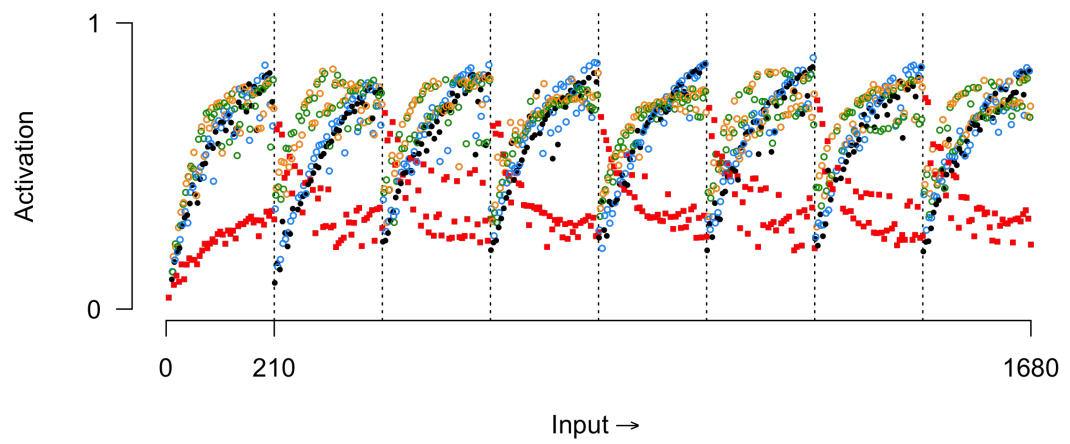

Cat-pos-HL [1] (note: cat. model)

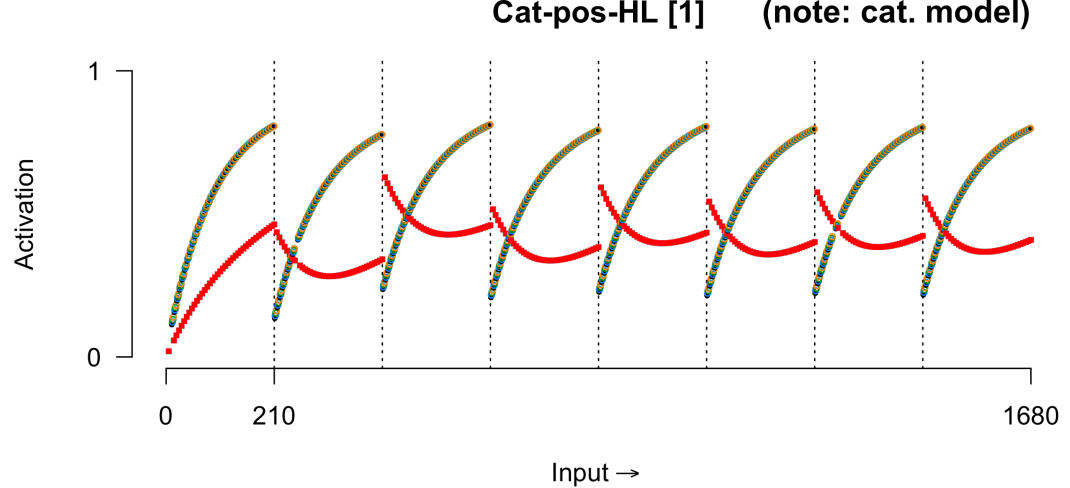
stimuli:

- $1 \circ 2 \circ 3 \circ 4$ - 5

Figure D1. Cluster 1: Activation values for five EDL simulations with strong correlations. Activation values are based on the experimental stimuli for one participant in the experiment. Cat-val-SD is included in Figure D2 and is included in this group due to similar patterns for stimuli 1-4., but it does not capture unlearning of the deviant, stimulus 
Cat-val-SD [1]

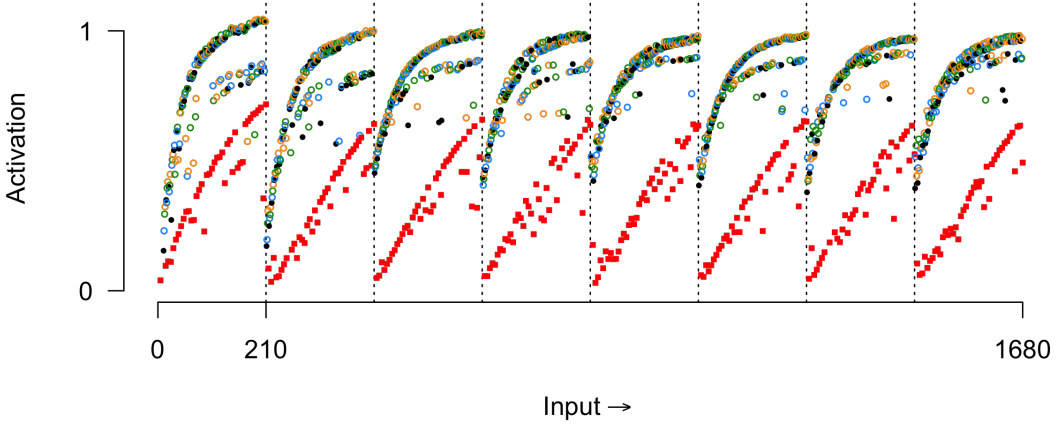

Cat-diff-HL

ভ
Cat-diff-SD

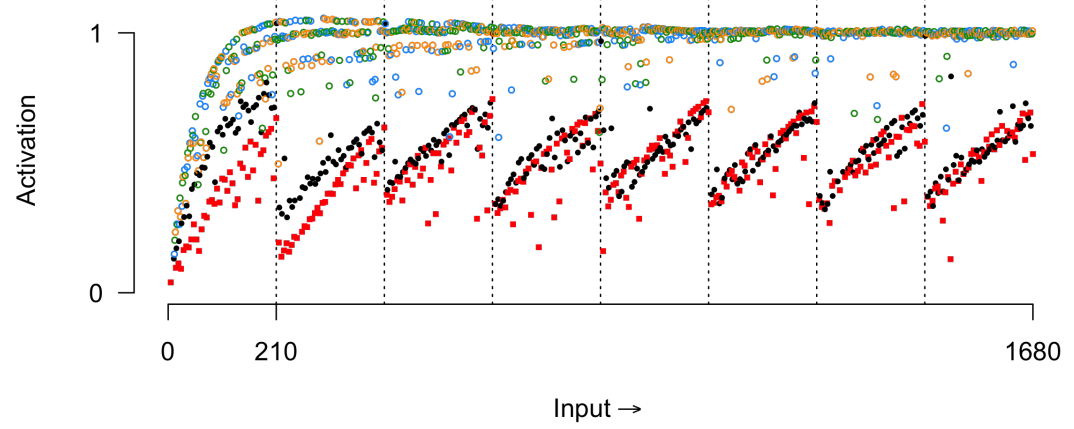

Pred-diff-SD

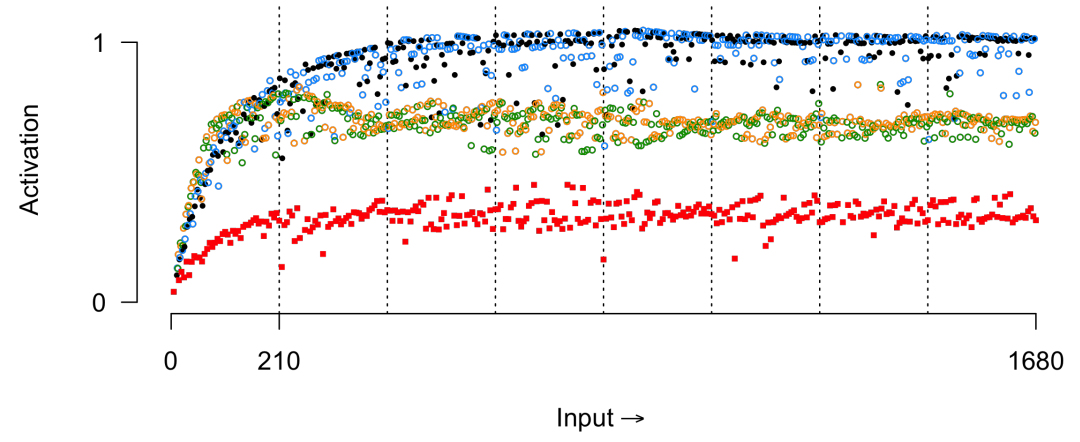

stimuli:

- $1 \circ 2 \circ 3 \circ 4$ - 5

Figure D2. Activation values for three other (not correlated) EDL simulations that outperform their baselines and the random models. Activation values are based on the experimental stimuli for one participant in the experiment. Note that model Cat-val-SD (topleft panel) is part of cluster 1, see Figure D1. 
Pred-bg-VAL [2]

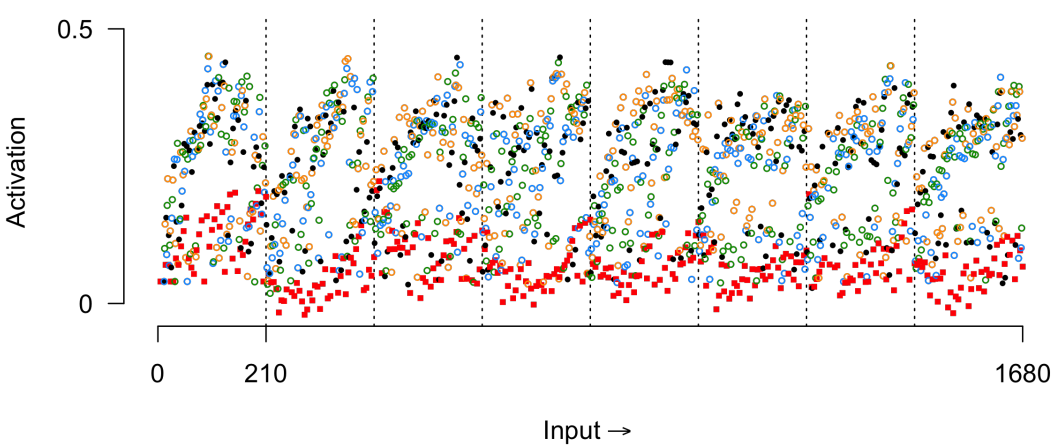

Pred-val-VAL [2]

ç

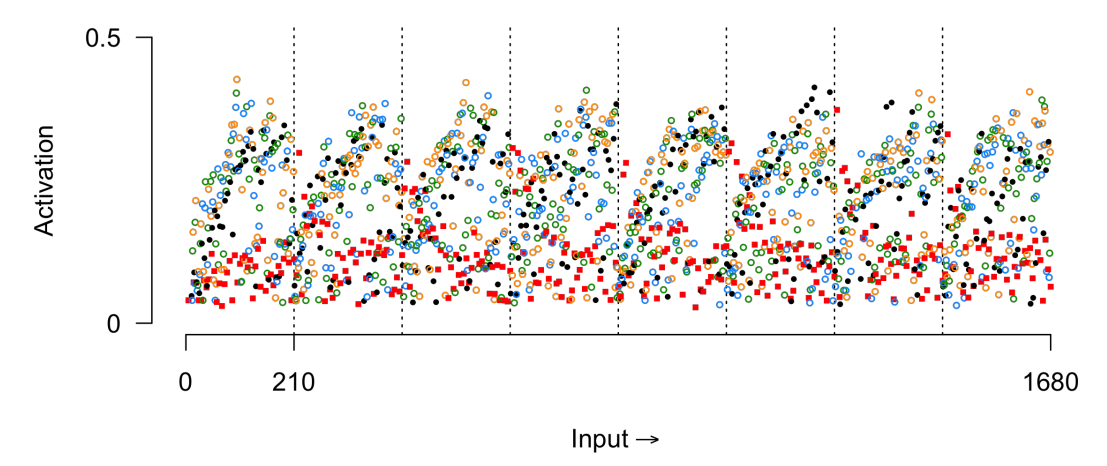

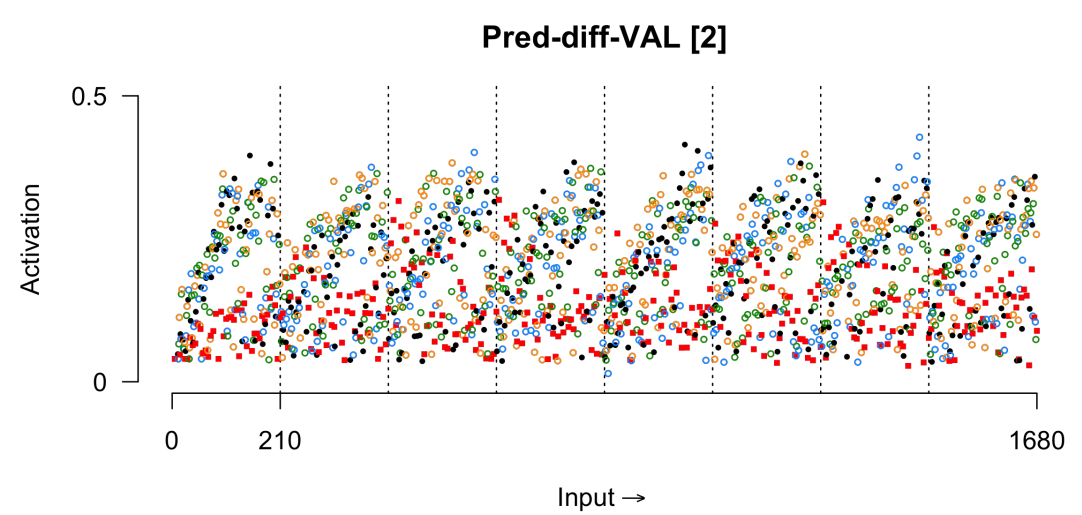

Input $\rightarrow$

stimuli:

- $1 \circ 2 \circ 3 \circ 4$ - 5

Figure D3. Cluster 2: Activation values for three EDL simulations with strong correlations. Activation values are based on the experimental stimuli for one participant in the experiment. Note that the range of activation values for these models is different from the EDL models presented in Figures D1 and D2. 


\section{Appendix E. TRFs}

\section{E.1. Baseline models}

Figures E2, E3, and E4 illustrate the TRFs for the baseline models High-Low (HL), Standard-Deviant (SD), and the Probability model for the ERP data in channels FC6 and Pz. For all baseline models the constant dimension contains a constant spike for each incoming stimulus (black solid line). The variable dimension differs between the three models:

- In the HL model (Figure E2) the variable dimension contains uniformly sized spikes for each high tone. The ERP of the high tones is fitted by the sum of the two dimensions, and hence, the variable dimension fits the difference between high and low tones and the constant dimension fits the ERP of the low tones.

- In the SD model (Figure E3) the variable dimension contains uniformly sized spikes for each deviant stimulus. The ERP of the deviant stimuli is fitted by the sum of the two dimensions, the variable dimension fits the standard stimuli, and the constant dimension fits the ERP of the difference between deviant and standard stimuli.

- In the Probability model (Figure E4) the variable dimension contains spikes that reflect the probability of the stimulus to occur (see Figure E1), based on the frequency of occurrence in the experiment. The probability values range between 0.012 and 0.179 . The ERP of the stimuli with a probability of 0.179 is fitted by the sum of the constant dimension and $0.179 *$ variable dimension.

\section{Stimulus probability}

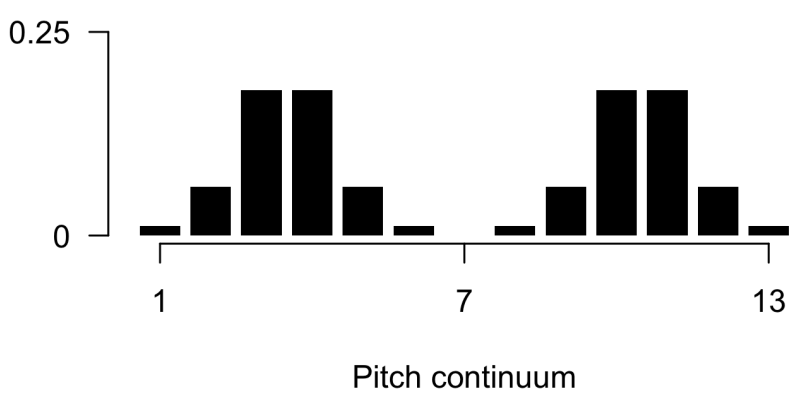

Figure E1. Stimulus frequency (in probability values) for all presented pitch values. 
TRFs baseline model High-Low

a. Early time window FC6

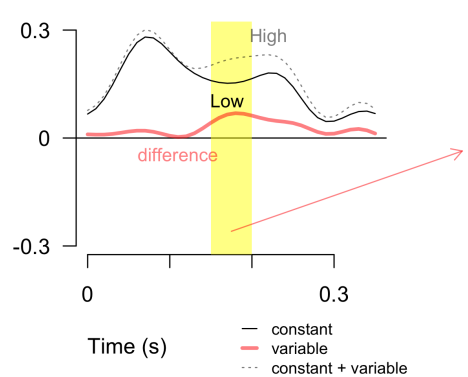

c. Early time window Pz

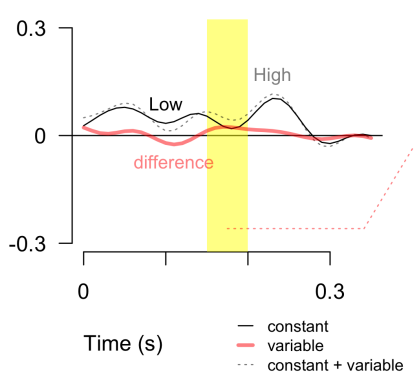

b. Late time window FC6

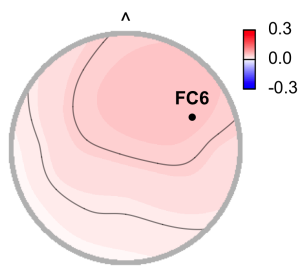

difference

$0.150-0.200 \mathrm{~s}$

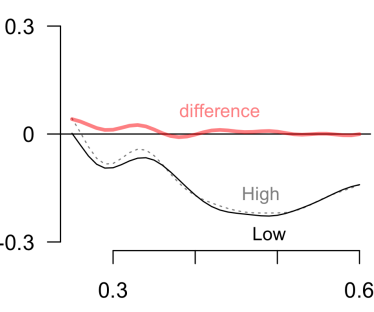

Time (s) d. Late time window $\mathrm{Pz}$

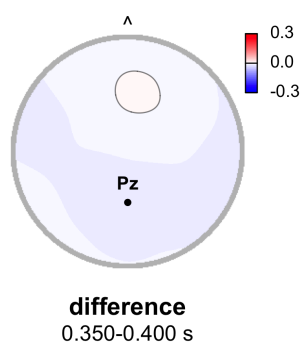

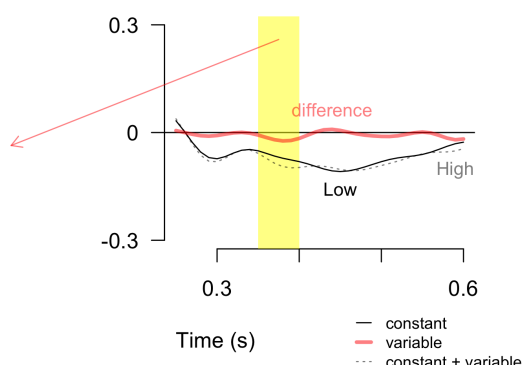

Figure E2. TRFs fitting the activations of baseline model High-Low to the ERPs in channels FC6 (top row) and $\mathrm{Pz}$ (bottom row). As the TRFs are fitted on z-scores of the ERP data, the TRF scale has arbitrary units. The black solid lines show the constant dimension (representing a low tone), the red thick lines show the variable dimension (representing difference between high and low tones), and the dotted lines show the sum of the constant and variable dimension (representing the high tones).

Panels $a$ and $c$ : TRFs early time window. Panels $b$ and $d$ : TRFs late time window. Note that only in the early time window the baseline model shows a small difference between high and low tones, around $0.175 \mathrm{~s}$ after stimulus onset, indicated by the yellow time window. Further, the TRFs do not predict any difference between high and low tones in channel $\mathrm{Pz}$. 


\section{TRFs baseline model Standard-Deviant}

a. Early time window FC6

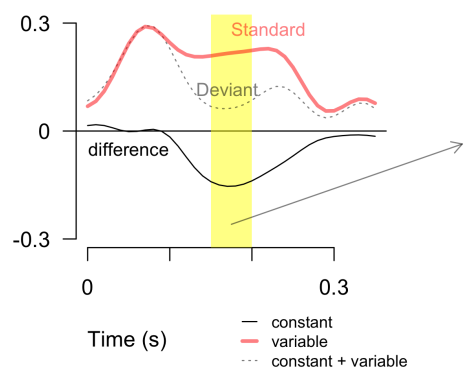

c. Early time window $\mathrm{Pz}$

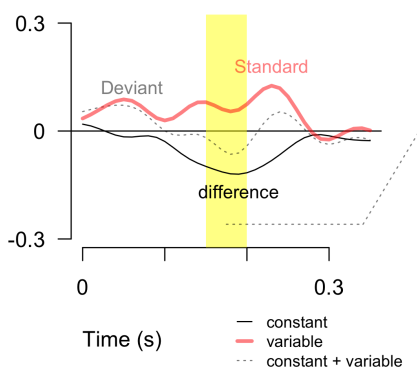

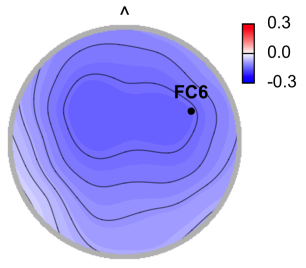

difference

$0.150-0.200 \mathrm{~s}$

b. Late time window FC6

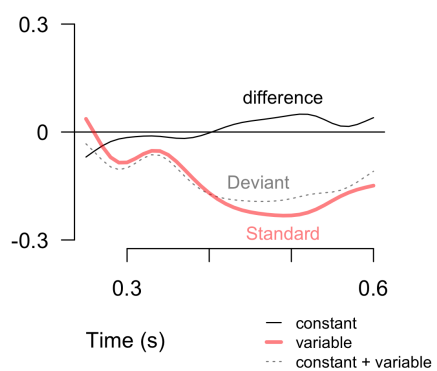

d. Late time window $\mathrm{Pz}$

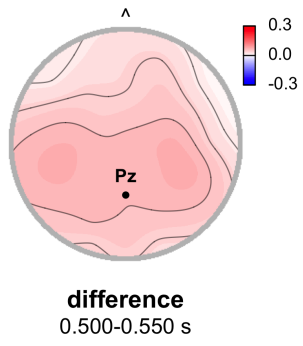

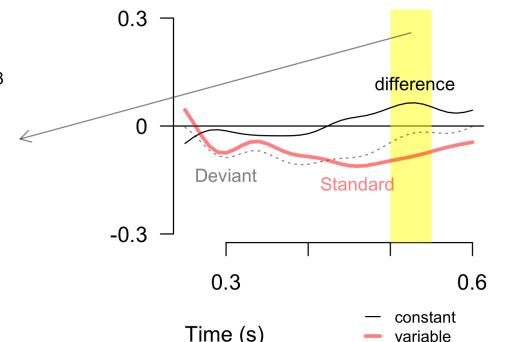

Time (s)
- constant

constant + variable

Figure E3. TRFs fitting the activations of baseline model Standard-Deviant to the ERPs in channels FC6 (top row) and Pz (bottom row). As the TRFs are fitted on z-scores of the ERP data, the TRF scale has arbitrary units. The black solid lines show the constant dimension (representing the difference between deviant and standard stimuli), the red thick lines show the variable dimension (representing the standard stimuli), and the dotted lines show the sum of the constant and variable dimension (representing the deviant stimuli).

Panels $a$ and $c$ : TRFs early time window. Panels $b$ and $d$ : TRFs late time window. Note that the difference between deviant and standard stimuli is much larger in the early time windows, peaking around $0.175 \mathrm{~s}$ after stimulus onset as indicated by the yellow time, than in the later time window. 


\section{TRFs Probability model}

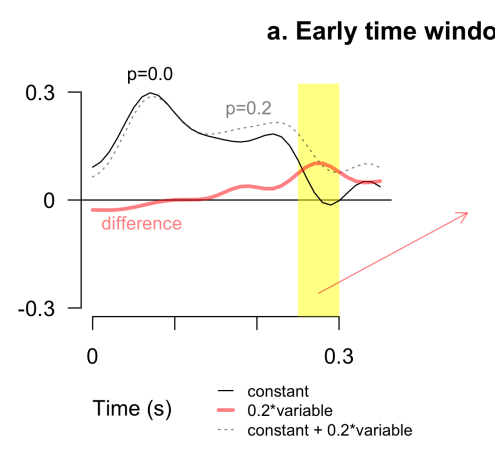

c. Early time window $\mathrm{Pz}$

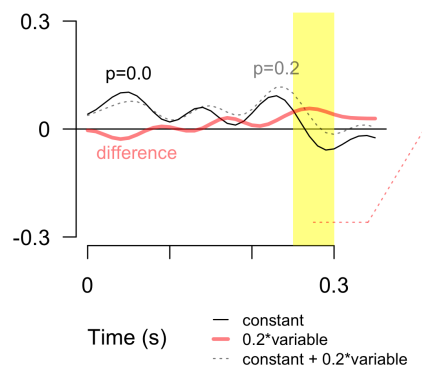

b. Late time window FC6

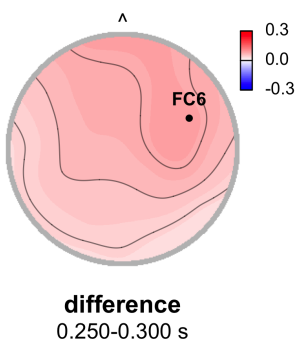

d. Late time window Pz

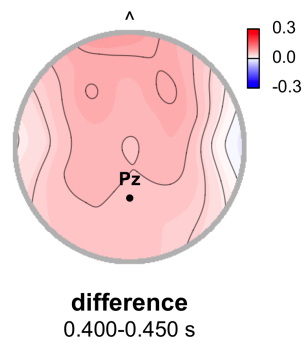

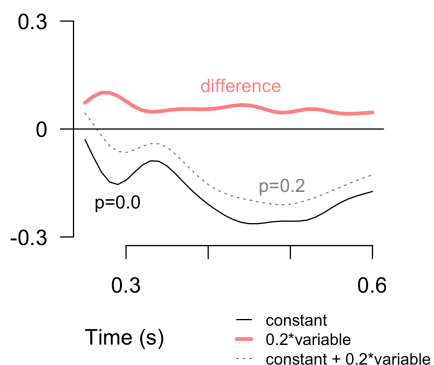

constant $+0.2^{*}$ variable

Figure E4. TRFs fitting the activations of the Probability model to the ERPs in channels FC6 (top row) and $\mathrm{Pz}$ (bottom row). As the TRFs are fitted on z-scores of the ERP data, the TRF scale has arbitrary units. The black solid lines show the constant dimension (representing the probability value 0.0), the red thick lines show the variable dimension (representing difference between probability values 0.2 and 0.0 ), and the dotted lines show the sum of the constant and variable dimension (representing the probability 0.2).

Panels $a$ and $c$ : TRFs early time window. Panels $b$ and $d$ : TRFs late time window. Note that the effect of probability starts only around $0.250 \mathrm{~s}$ after stimulus onset, and continues in the later time window. 


\section{E.2. EDL models}

The TRF for EDL model Pred-bg-HL (Figure 9) was discussed in Section 6. The TRFs of EDL models Pred-val-HL, Pred-diff-HL, and Cat-pos-HL are similar to the TRFs of model Pred-bg-HL and therefore not included in this section. Below the TRFs for the other models are included: Cat-diff-HL, Cat-val-SD, Cat-diff-SD, and Pred-bg-VAL. The TRFs of the models are Pred-val-VAL and Pred-diff-VAL are similar to the TRF of Pred-bg-VAL, and therefore not included.

\section{TRFs EDL model Cat-diff-HL}

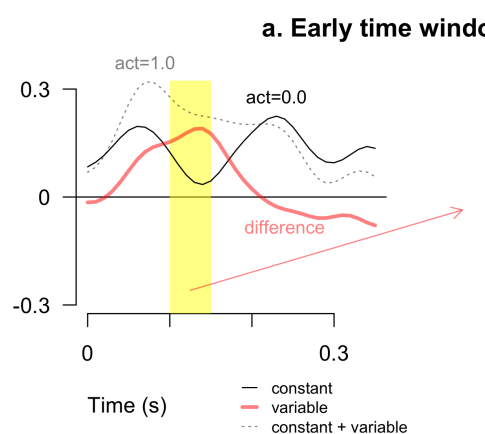

c. Early time window Pz

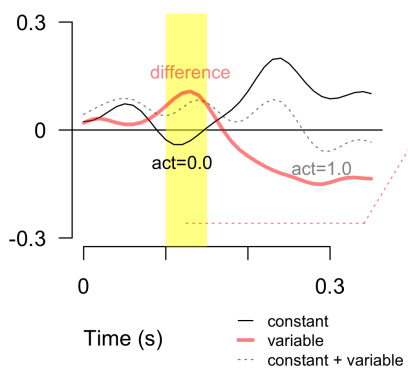

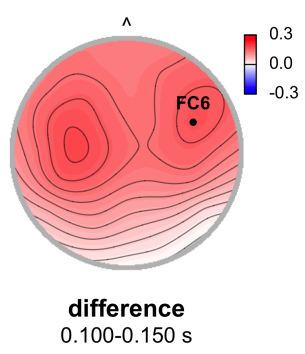
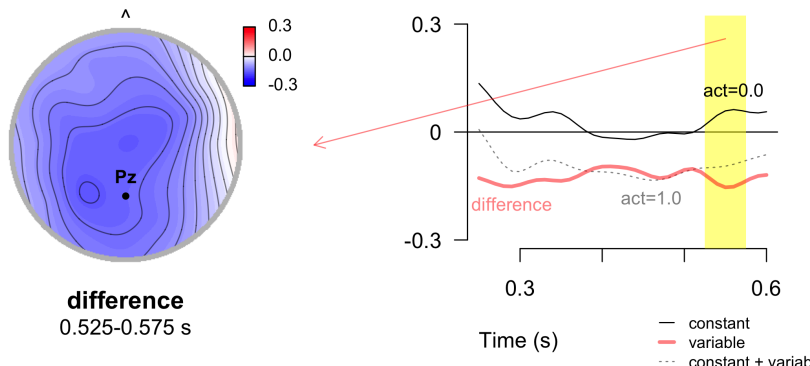

Figure E5. TRFs fitting the activations of EDL model Cat-diff-HL to the ERPs in channels FC6 (top row) and $\mathrm{Pz}$ (bottom row). As the TRFs are fitted on z-scores of the ERP data, the TRF scale has arbitrary units. The black solid lines show the constant dimension (representing an activation value 0.0), the red thick lines show the variable dimension (representing difference between the activation values 1.0 and 0.0 ), and the dotted lines show the sum of the constant and variable dimension (representing the activation value 1.0).

Panels $a$ and $c$ : TRFs early time window. The difference is predicted to peak around $0.125 \mathrm{~s}$ after stimulus onset, indicated by the yellow time window. Panels $b$ and $d$ : TRFs late time window. The EDL model only beats its baseline in channel Pz, but not in FC6. The scalp plot in panel a shows the distribution of the difference in the early yellow marked time window. The scalp plot in panel $d$ shows the distribution of the difference in the later yellow marked time window. 
TRFs EDL model Cat-val-SD

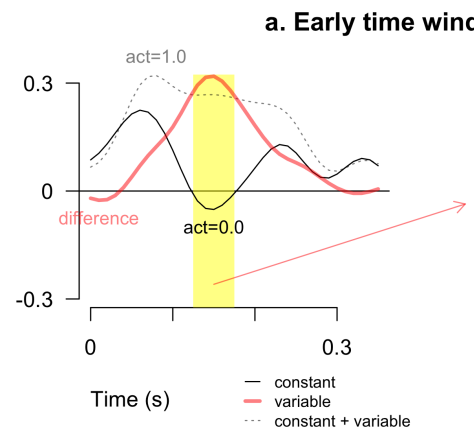

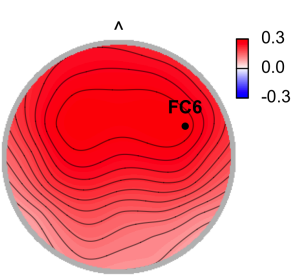

difference $0.125-0.175 \mathrm{~s}$ b. Late time window FC6

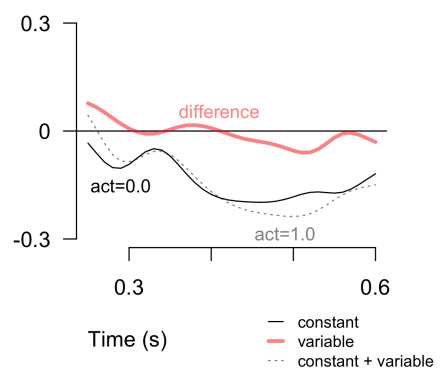

c. Early time window Pz

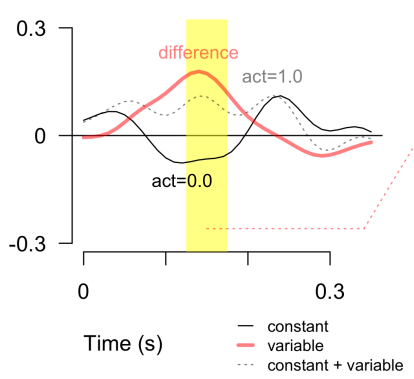

d. Late time window $\mathrm{Pz}$
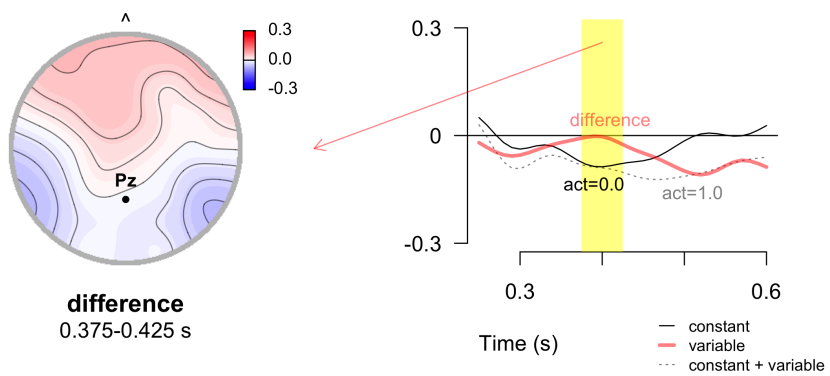

Figure E6. TRFs fitting the activations of EDL model Cat-val-SD to the ERPs in channels FC6 (top row) and $\mathrm{Pz}$ (bottom row). As the TRFs are fitted on z-scores of the ERP data, the TRF scale has arbitrary units. The black solid lines show the constant dimension (representing an activation value 0.0), the red thick lines show the variable dimension (representing difference between the activation values 1.0 and 0.0 ), and the dotted lines show the sum of the constant and variable dimension (representing the activation value 1.0).

Panels $a$ and $c$ : TRFs early time window. The difference is predicted to peak around $0.125 \mathrm{~s}$ after stimulus onset, indicated by the yellow time window. Panels $b$ and $d$ : TRFs late time window. The EDL model only beats its baseline in channel Pz, but not in FC6. The scalp plot in panel a shows the distribution of the difference in the early yellow marked time window. The scalp plot in panel $d$ shows the distribution of the difference in the later yellow marked time window. 


\section{TRFs EDL model Cat-diff-SD}

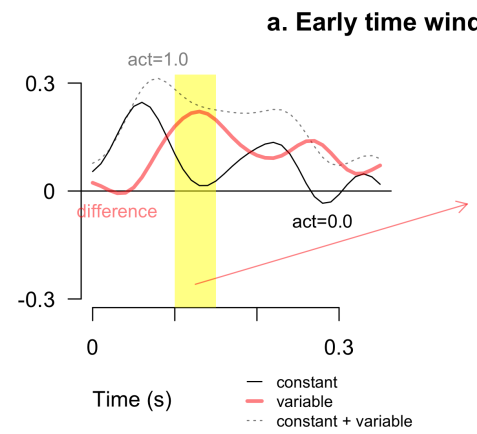

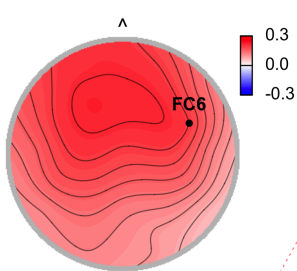

difference $0.100-0.150 \mathrm{~s}$ b. Late time window FC6

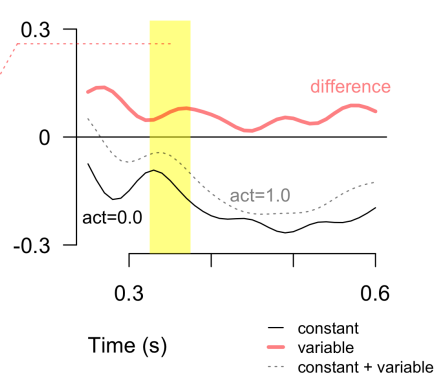

c. Early time window Pz

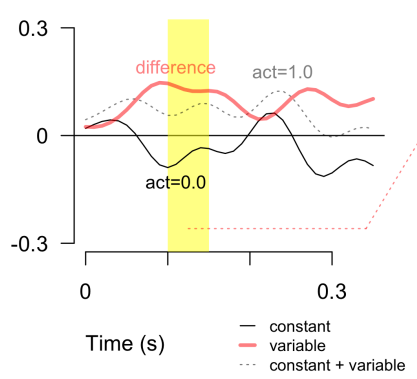

d. Late time window $\mathrm{Pz}$
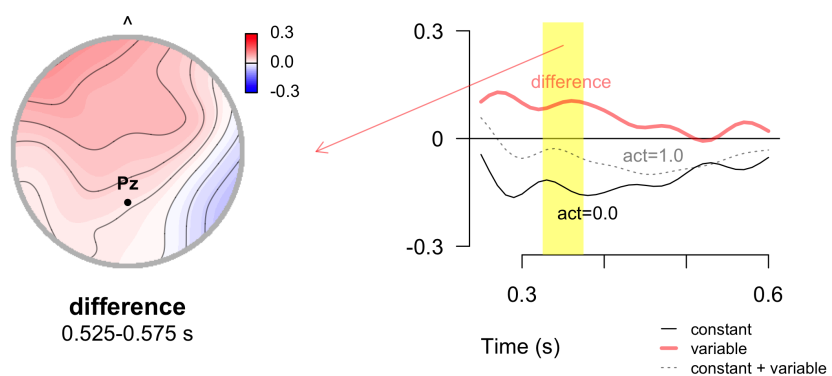

Figure E7. TRFs fitting the activations of EDL model Cat-diff-SD to the ERPs in channels FC6 (top row) and $\mathrm{Pz}$ (bottom row). Note that the EDL model does not beat its baseline in these channels, see Figure7, but we choose FC6 and Pz for comparison with other EDL models. As the TRFs are fitted on z-scores of the ERP data, the TRF scale has arbitrary units. The black solid lines show the constant dimension (representing an activation value 0.0), the red thick lines show the variable dimension (representing difference between the activation values 1.0 and 0.0 ), and the dotted lines show the sum of the constant and variable dimension (representing the activation value 1.0).

Panels $a$ and $c$ : TRFs early time window. Panels $b$ and $d$ : TRFs late time window. The scalp plot in panel $a$ shows the distribution of the difference in the early yellow marked time window. The scalp plot in panel $d$ shows the distribution of the difference in the later yellow marked time window. 


\section{TRFs EDL model Pred-bg-VAL}

a. Early time window FC6

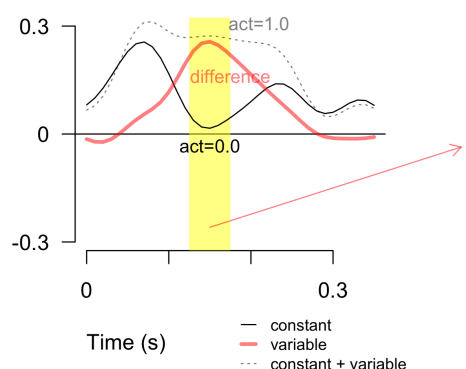

c. Early time window $\mathrm{Pz}$

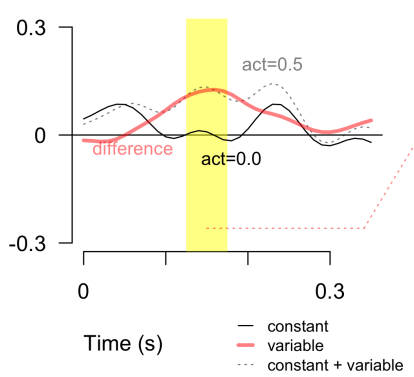

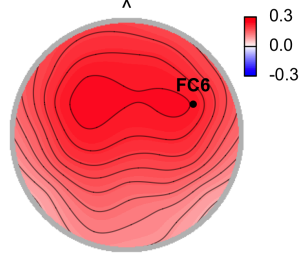

difference $0.125-0.175 \mathrm{~s}$ b. Late time window FC6

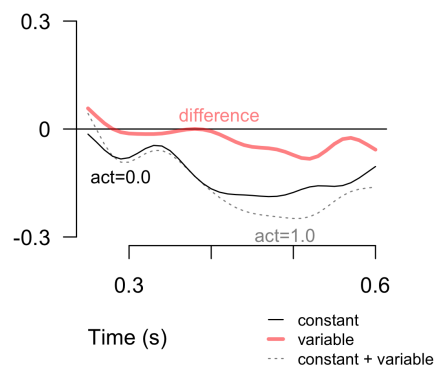

d. Late time window $\mathrm{Pz}$

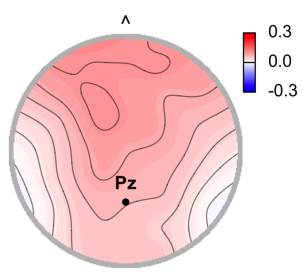

difference

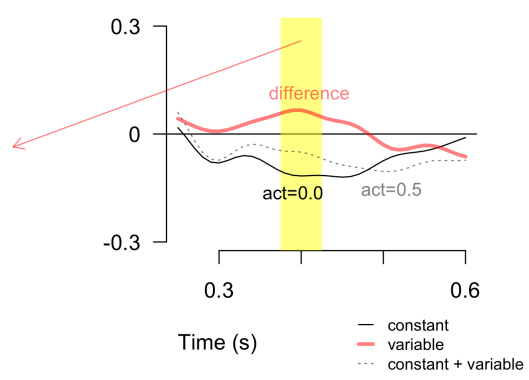

Figure E8. TRFs fitting the activations of EDL model Pred-bg-VAL to the ERPs in channels FC6 (top row) and $\mathrm{Pz}$ (bottom row). As the TRFs are fitted on z-scores of the ERP data, the TRF scale has arbitrary units. The black solid lines show the constant dimension (representing an activation value 0.0), the red thick lines show the variable dimension (representing difference between the activation values 0.5 and 0.0 ), and the dotted lines show the sum of the constant and variable dimension (representing the activation value 0.5). Note that the range of activation values is smaller than for the other EDL models, therefore the variable dimension was adjusted to reflect the maximum activation (see Figure D3).

Panels $a$ and $c$ : TRFs early time window. Panels $b$ and $d$ : TRFs late time window. The scalp plot in panel $a$ shows the distribution of the difference in the early yellow marked time window. The scalp plot in panel $d$ shows the distribution of the difference in the later yellow marked time window. 